\title{
Spectroscopic monitoring of the jet in the symbiotic star MWC 560^
}

\section{Spectroscopic properties, general outflow structure and system parameters}

\author{
H. M. Schmid ${ }^{1, \star \star}$, A. Kaufer ${ }^{2}$, M. Camenzind ${ }^{1}$, Th. Rivinius ${ }^{3}$, O. Stahl ${ }^{1}$, T. Szeifert ${ }^{2}$, \\ S. Tubbesing ${ }^{1}$, and B. Wolf ${ }^{1}$
}

1 Landessternwarte Heidelberg-Königstuhl, 69117 Heidelberg, Germany

2 European Southern Observatory, Alonso de Cordova 3107, Santiago 19, Chile

3 European Southern Observatory, Karl-Schwarzschild-Str. 2, 85748 Garching bei München, Germany

Received 11 June 2001 / Accepted 24 July 2001

\begin{abstract}
We present the results of an intensive monitoring program of the jet absorptions in the symbiotic system MWC 560, obtained with the FEROS echelle spectrograph at the ESO $1.5 \mathrm{~m}$ Telescope. MWC 560 is a unique jet source because the line of sight lies practically parallel to the jet axis so that the outflowing gas is seen as absorption in the continuum of the accreting object, in the emission line spectrum of the accretion disk and temporary also in the spectrum of the red giant companion. Highly variable, blue-shifted jet absorption components, due to Hi, HeI, NaI, CaII and FeII are observed, which are detached from the undisplaced, narrow emission line components. The allowed emission lines from neutral and singly ionized heavy elements vary simultaneously with the strongly variable continuum emission. Therefore they can be attributed to the irradiated (chromospheric) layers of the neutral part of the accretion disk. The fluxes of forbidden emission lines are practically constant because they originate in a much larger volume. The structure and variability of the jet absorptions indicate the presence of three distinct outflow regions along the jet axis: I. An initial acceleration region above the disk with low velocities $<600 \mathrm{~km} \mathrm{~s}^{-1}$ which covers only partly the central continuum source; II. A highly variable outflow region covering the continuum source and up to about half of the line emission from the disk. This region shows repeatedly high velocity components $\approx 1800-2500 \mathrm{~km} \mathrm{~s}^{-1}$ which are decelerated to $<1500 \mathrm{~km} \mathrm{~s}^{-1}$ within one to a few days. The appearance of high velocity components is anti-correlated and therefore closely related to the low velocity absorptions of region I. The life time of the high velocity components suggests that region II extends to about one to a few AU from the jet source; III. A steady flow at an intermediate velocity of $\approx 900-1300 \mathrm{~km} \mathrm{~s}^{-1}$ at a distance of the order $\sim 10 \mathrm{AU}$ from the jet source. This component covers the hot continuum source and the entire narrow line region of the accretion disk. At the beginning of our campaign region III covers also the extended red giant companion with two absorption components at $1250 \mathrm{~km} \mathrm{~s}^{-1}$ and $1140 \mathrm{~km} \mathrm{~s}^{-1}$, which can be considered as terminal velocities $v_{\infty}$ of the jet outflow. The components disappear during the following several weeks as expected for the end of an occultation phase of the red giant by the collimated jet occuring probably regularly once per binary orbit. Several fast moving (1300-1700 $\mathrm{km} \mathrm{s}^{-1}$ ), narrow absorptions are present in the Ca II resonance lines. The high speed, low column density, and the long life time ( months) suggest that these are radiative bow shocks in the jet cocoon generated by the collision of the transient high velocity components with slower moving jet material. A geometric model for the jet outflow in MWC 560 is presented. System parameters are derived based on our spectroscopic data and previous studies. Beside other parameters a binary separation of the order $4 \mathrm{AU}$, a jet inclination of $<16^{\circ}$, a mass accretion rate of $5 \times 10^{-7} M_{\odot} \mathrm{yr}^{-1}$ and a jet outflow rate larger than $7 \times 10^{-9} M_{\odot} \mathrm{yr}^{-1}$ are derived.
\end{abstract}

Key words. ISM: jets and outflows - binaries: symbiotic - circumstellar matter - stars: mass loss - stars: individual: MWC $560=$ V694 Mon

Send offprint requests to: H. M. Schmid,

e-mail: schmid@astro.phys.ethz.ch

* Based on observations obtained with FEROS at the ESO

$1.5 \mathrm{~m}$ Telescope at La Silla, Chile (ESO program 62.H-0319).

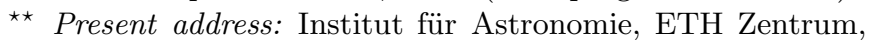
8092 Zürich, Switzerland.

\section{Introduction}

The peculiar nature of MWC 560 (V694 Mon) was first reported in the catalogue of Be and Ae stars by Merrill \& Burwell (1943). They noticed emission lines of HI and CaII K with absorption components on their blue sides displaced by about $1000 \mathrm{~km} \mathrm{~s}^{-1}$. Balmer emission 
and blue shifted absorptions were also found to be present about thirty years later by Sanduleak \& Stephenson (1973). They detected also TiO bands and classified therefore the object as M4ep for peculiar M-type emission line star. Due to the emission lines, the blue continuum and the red giant absorptions MWC 560 is often classified as symbiotic binary. A short report of dedicated observations was later given by Bond et al. (1984). They found that the blue shifted absorption components have a complex structure extending to $-3000 \mathrm{~km} \mathrm{~s}^{-1}$ which varied strongly on time scales of one day. An IUE spectrum showed a strong UV continuum with a low-excitation absorption spectrum, and high-speed photometry revealed flux variations of $0.2 \mathrm{mag}$ on a time scale of a few min.

MWC 560 underwent in early 1990 a photometric outburst of about 2 mag. Spectra taken during this phase by Tomov et al. (1990) display spectacular spectral changes in the blue shifted absorption components of H I and Ca II. Tomov et al. (1990) proposed that a jet outflow seen along the line of sight could explain the observations. This publication triggered many new observational studies of MWC 560. The spectroscopic observations during the outburst maximum between January and April 1990 showed strongly variable outflow components having sometimes radial velocities $(R V)$ beyond $R V<-4000 \mathrm{~km} \mathrm{~s}^{-1}$ and sometimes low velocity around $R V \approx-500 \mathrm{~km} \mathrm{~s}^{-1}$. The maximum outflow velocity measured for an absorption component was $-6000 \mathrm{~km} \mathrm{~s}^{-1}$ for March 14, 1990 (Tomov et al. 1990; Buckley 1992; Tomov \& Kolev 1997). IUE spectra taken between February and April 1990 by Michalitsianos et al. (1991) revealed the same $R V$-variability in low excitation absorption lines in the UV region. In addition strong short term variations (flickering) of the hot continuum were measured.

During the following observing season from September 1990 to March 1991 the velocity of the absorption components has dropped to about $-300 \mathrm{~km} \mathrm{~s}^{-1}$ (Tomov \& Kolev 1997; Buckley 1992). At the same time the UV continuum was almost entirely attenuated by a curtain of innumerable absorption lines of neutral and ionized heavy elements in a dense outflow (Maran et al. 1991; Shore et al. 1994).

In September 1991 the absorption components have reached again a higher velocity of about $-2000 \mathrm{kms}^{-1}$. Since then the strong Balmer lines, e.g., $\mathrm{H} \delta, \mathrm{H} \gamma$ and $\mathrm{H} \beta$ showed always a variable but persistent, deep and broad absorption trough around $R V \approx-1500 \mathrm{~km} \mathrm{~s}^{-1}$ with occasional extensions to $-3000 \mathrm{kms}^{-1}$ (Tomov \& Kolev 1997; Mikolajewski et al. 1997; Panferov et al. 1997; Iijima 2000). This seems to be the "normal" type of outflow for the MWC 560 system as the same type of absorptions was already reported for the pre-outburst observations mentioned above. We are not aware of any spectroscopic observations taken before the 1990 outburst or after September 1991 where no strongly blue-shifted $\left(R V<-1000 \mathrm{kms}^{-1}\right)$, deep and broad absorptions were visible for the strong Balmer lines $\mathrm{H} \delta, \mathrm{H} \gamma$ or $\mathrm{H} \beta$. Thus the outburst has interrupted the persistent outflow from the MWC 560 system with a phase of very high velocity ejections in early 1990, followed by a phase with a high density but slow outflow which lasted about a year.

The brightness of the MWC 560 decreased after the outburst maximum of about $m_{V}=9.2 \mathrm{mag}$ in mid-1990 to $\approx 10 \mathrm{mag}$ in mid-1991 and $\approx 10.5 \mathrm{mag}$ in 1993 . Since then the object remained at a level around 10.5-11.0 mag (e.g., Tomov et al. 1996; Mikolajewski et al. 1997). Before the 1990 outburst the brightness of MWC 560 varied since about 1930 in the range $m_{B}=11.0-12.5 \mathrm{mag}$ and was never brighter than 10.5 mag (Luthardt 1991; Doroshenko et al. 1993). Thus, the 1990 outburst is the brightest phase recorded for this object.

Short term flickering variability of $\Delta m \approx 0.2-0.4 \mathrm{mag}$ on time scales of 10-60 min was measured for the hot component before, during and after the 1990 outburst (Bond et al. 1984; Tomov et al. 1990, 1996; Michalitsianos et al. 1991, 1993; Dobrzycka et al. 1996; Ishioka et al. 2001). It seems that the strong flickering of the hot component is always present in MWC 560.

Jets and MWC 560. The spectral properties of the blue shifted absorptions are usually interpreted as jet outflow seen along the jet axis as first suggested by Tomov et al. (1990). The structure of the lines differs distinctively from classical P Cygni line profiles for a stellar wind. Compared to stellar winds, there is a lack of absorption at low velocities pointing to an outflow geometry, where the acceleration region is not in front of the dominating radiation source. The maximum outflow speed of $-6000 \mathrm{~km} \mathrm{~s}^{-1}$ exceeds also the speed of nova ejecta by about a factor of two and is far beyond velocities observed in any other symbiotic system. Also the strong variability in the absorption structure is indicative that the mass outflow differs completely from a radiatively driven stellar wind.

Based on these consideration the jet hypothesis is very reasonable for the interpretation of the outflow from MWC 560. Jets are observed in many astrophysical objects, like pre-main sequence stars (e.g., Mundt \& Fried 1983), binaries with compact objects, like SS 433 or black hole binaries (e.g., Margon 1984; Mirabel \& Rodriguez 1999), supersoft X-ray sources (e.g., Cowley et al. 1998), and of course in active galactic nuclei. All these systems have an accretion disk which is considered to be a necessary condition for jet outflows. Further, it is widely believed that a rotating magnetosphere is required for accelerating and collimating the jet outflow from these systems (e.g., Camenzind 1997).

MWC 560 has the required properties for a jet source. It is a symbiotic binary system, where a late M giant transfers matter onto a companion. Orbital periods for symbiotic systems are several hundred days or longer. Thus the combination of a giant companion undergoing heavy mass loss and the long orbital period allow that an extended accretion disk with an outer radius $r \approx 100 R_{\odot}$ and a high mass transfer rate of $\approx 10^{-7} M_{\odot} \mathrm{yr}^{-1}$ can be present in these systems. The flickering activity can thereby be interpreted as accretion phenomenon at the inner disk 
boundary as observed in many cataclysmic variables. The outburst is probably caused by an accretion disk instability similar to dwarf novae or FU Ori stars. In most symbiotic systems the accreting object is a white dwarf. The flickering time scale and the outflow velocities measured for MWC 560 suggest that also this symbiotic binary harbors an accreting white dwarf.

MWC 560 is not alone among the symbiotic systems in having a jet outflow. $\mathrm{CH}$ Cyg is famous for having produced a well observed two-sided jet after a distinct drop in visual brightness (Taylor et al. 1986). Or, the suspected symbiotic system He2-90 exhibits a highly collimated, twosided optical jet with more than five pairs of emission knots located symmetrically on either side of the source (Sahai \& Nyman 2000).

However, MWC 560 is an extraordinary jet source because the line of sight lies practically parallel to the jet axis. Given the small opening angle for jets of about $\sim 10^{\circ}$ only a tiny fraction $(\sim 1 \%)$ of all jet sources offer the possibility to have the line of sight to the jet source within the jet. To our knowledge MWC 560 is the only stellar jet source known, where this is the case. This very special configuration offers the unique opportunity to obtain complementary information about the physics involved in the acceleration and collimation of stellar jets. Therefore we performed for MWC 560 an intensive monitoring program with high resolution spectroscopy in order to investigate the dynamics of the jet gas near the central source.

In this paper we describe the properties of the different spectral components and derive physical parameters and a geometric model for the MWC 560 system. In a forthcoming study we will concentrate on the geometry and dynamics of the jet acceleration region.

\section{Observations}

MWC 560 has been intensively monitored with FEROS at the ESO $1.5 \mathrm{~m}$ telescope. FEROS is a new generation fiber fed echelle spectrograph, providing a high resolution of $R=48000$, a wide wavelength coverage from about $3700 \AA$ to $9000 \AA$ in one exposure and a high detective efficiency (Kaufer et al. 1999, 2000). The 39 orders of the echelle spectrum are registered with a $2 \mathrm{k} \times 4 \mathrm{k}$ EEV CCD with excellent quantum efficiency. The spectrograph was built for ESO by the FEROS consortium of the Landessternwarte Heidelberg and the Astronomical Observatory Copenhagen.

The present data have been collected from Nov. 1998 to Jan. 1999 during commissioning time and guaranteed observing time (the latter for building FEROS). In total 86 echelle spectra have been secured during two series of 26 and 31 consecutive nights. Only during one night no data could be obtained due to bad weather. In 22 nights two or more spectra were taken in order to assess spectroscopic changes on timescales of $\sim$ hours. Table 1 gives a log of the observations. With the typical exposure time of $15 \mathrm{~min}$ a signal-to-noise ratio of $S / N \approx 30$ is obtained for MWC 560 from about $4200 \AA$ to $7500 \AA$ and somewhat less at shorter and longer wavelengths.

A fraction of our echelle spectra could in principle be flux calibrated using the spectra of standard stars observed during the same night, provided the data were taken under photometric conditions. For a relative flux estimate of all spectra it is more advantageous to use the relative strength of the spectral features from the red giant as "flux calibration source", assuming that the red giant spectrum is constant during our campaign (see Sect. 2.4).

All spectra are reduced using the dedicated FEROS data reduction software implemented in the ESO-MIDAS system. The spectra are stored in a on-line searchable data base at the Landessternwarte in Heidelberg. Part of these spectra, those taken during the commissioning period, are public and retrievable via Internet (see http://www.lsw.uni-heidelberg.de).

\subsection{A mean spectrum of MWC 560}

Figure 1 shows a mean spectrum of MWC 560 from $3750 \AA$ to $6750 \AA$. The predominant features in the spectrum are:

- the broad, blue shifted absorptions called in this paper the jet absorptions;

- the numerous narrow emission lines from singly ionized and neutral heavy elements;

- the strong Balmer emission lines;

- the featureless blue continuum (apart from the jet absorptions);

- the molecular bands of the red giant in the red spectral region.

The mean spectrum plotted in Fig. 1 is constructed from about half the spectra obtained in our campaign. The spectra are selected according to their good signal-to-noise in the 3750 to $3850 \AA$ range in order to obtain a high quality mean spectrum down to $3750 \AA$. The $S / N$-ratio per $0.03 \AA$ pixel of the resulting spectrum is about 50 at $3800 \AA$, well above 100 from $4000 \AA$ to $7000 \AA$, and slightly below 100 for longer wavelengths up to the end of the extracted spectrum at $8900 \AA$.

All spectra have been normalized by fitting a spline function to continuum points spaced roughly by $100 \AA$. Determining the continuum level is not everywhere unambiguous. In the region $3750 \AA$ to $4000 \AA$ there are many broad absorption lines and innumerable narrow emission lines making a continuum determination difficult. Another problem in this range is the instrument response curve which decreases strongly towards the UV. For longer wavelengths it is easy to find line free continuum regions for the normalization. For the red portion of the spectra we have chosen the continuum points in regions where no strong discontinuities due to the $\mathrm{TiO}$ bands from the cool giant are present.

The presence of several bad CCD columns (cosmetics) requires a careful assessment of the $2 \mathrm{~d}$ frames for the identification of spurious features in the extracted spectrum. Regions where bad CCD columns affect the spectrum are 
Table 1. Log for the MWC 560 observing campaign from Nov. 14, 1998 (day 131) to Jan. 23, 1999 (day 201). Day is the integer of MJD - 51000 (MJD = modified Julian Date), UT the universal time (in hours:minutes) of the exposure start time, $t_{\exp }$ the exposure time in seconds, and "spec" the file number in the FEROS archive.

\begin{tabular}{|c|c|c|c|c|}
\hline day & & UT & $t_{\exp }$ & spec \\
\hline \multirow[t]{2}{*}{131} & $\mathrm{~A}$ & $05: 39$ & 900 & o07871 \\
\hline & B & $05: 57$ & 2400 & o07881 \\
\hline \multirow[t]{3}{*}{132} & A & $04: 56$ & 900 & $\mathrm{o} 08281^{a}$ \\
\hline & B & $05: 35$ & 900 & o08291 ${ }^{a}$ \\
\hline & $\mathrm{C}$ & $05: 54$ & 900 & $\mathrm{o} 08301^{a}$ \\
\hline 133 & & $05: 21$ & 1200 & o08861 \\
\hline 134 & & $07: 12$ & 900 & o09521 \\
\hline \multirow[t]{2}{*}{135} & $\mathrm{~A}$ & $06: 48$ & 1200 & o10051 \\
\hline & B & $08: 52$ & 900 & o10141 \\
\hline 136 & & $06: 13$ & 900 & o10611 \\
\hline 137 & & $08: 47$ & 900 & o11571 \\
\hline \multirow[t]{4}{*}{138} & $\mathrm{~A}$ & $06: 18$ & 900 & o11981 \\
\hline & B & $06: 36$ & 180 & o11991 ${ }^{b}$ \\
\hline & $\mathrm{C}$ & $06: 42$ & 180 & o12001 ${ }^{b}$ \\
\hline & $\mathrm{D}$ & $06: 48$ & 180 & o12011 \\
\hline 139 & & $06: 40$ & 900 & o12451 \\
\hline 140 & & 07:04 & 900 & o12921 \\
\hline 141 & & $07: 34$ & 1200 & $\mathrm{o} 13331^{a}$ \\
\hline 142 & & $03: 36$ & 900 & o13661 \\
\hline \multirow[t]{2}{*}{143} & A & $03: 37$ & 900 & o14161 \\
\hline & B & $08: 43$ & 900 & o14311 \\
\hline \multirow[t]{2}{*}{144} & A & $05: 23$ & 900 & o14641 \\
\hline & B & 08:44 & 900 & o14761 \\
\hline \multirow[t]{2}{*}{145} & A & $05: 44$ & 900 & o15121 \\
\hline & B & $08: 27$ & 900 & o15231 \\
\hline 146 & & 06:12 & 900 & o15551 \\
\hline \multirow[t]{2}{*}{147} & A & $05: 44$ & 900 & o15991 \\
\hline & B & $07: 13$ & 900 & o16051 \\
\hline \multirow[t]{3}{*}{148} & $\mathrm{~A}$ & $04: 22$ & 900 & o16341 \\
\hline & B & $06: 00$ & 900 & o16401 \\
\hline & $\mathrm{C}$ & $06: 31$ & 900 & o16421 \\
\hline
\end{tabular}

\begin{tabular}{|c|c|c|c|c|}
\hline day & & $\mathrm{UT}$ & $t_{\exp }$ & spec \\
\hline \multirow[t]{3}{*}{149} & A & 05:06 & 900 & o16751 \\
\hline & B & $06: 29$ & 1200 & o16801 \\
\hline & $\mathrm{C}$ & 08:36 & 900 & o16931 \\
\hline \multirow[t]{3}{*}{150} & A & $04: 33$ & 900 & o17171 \\
\hline & B & 05:39 & 900 & o17211 \\
\hline & $\mathrm{C}$ & $07: 45$ & 900 & o17331 \\
\hline \multirow[t]{3}{*}{151} & A & $05: 20$ & 900 & o17621 \\
\hline & B & $06: 46$ & 900 & o17681 \\
\hline & $\mathrm{C}$ & 08:05 & 900 & o17721 \\
\hline \multirow[t]{2}{*}{152} & $\mathrm{~A}$ & $04: 15$ & 900 & o18001 \\
\hline & B & 05:51 & 900 & o18091 \\
\hline 153 & & 07:09 & 900 & o18811 \\
\hline 154 & & $06: 35$ & 900 & o19181 \\
\hline 155 & & $05: 45$ & 900 & o19551 \\
\hline \multirow[t]{2}{*}{156} & $\mathrm{~A}$ & $03: 46$ & 900 & o19861 \\
\hline & B & 06:48 & 900 & o19961 \\
\hline
\end{tabular}

\begin{tabular}{|c|c|c|c|c|}
\hline day & & $\mathrm{UT}$ & $t_{\exp }$ & spec \\
\hline \multirow[t]{2}{*}{180} & $\mathrm{~A}$ & 07:04 & 600 & o25571 \\
\hline & B & $08: 46$ & 480 & o25621 \\
\hline 181 & & $03: 30$ & 600 & o25851 \\
\hline \multirow[t]{2}{*}{182} & $\mathrm{~A}$ & $03: 47$ & 600 & o26151 \\
\hline & B & $07: 20$ & 600 & o26221 \\
\hline 183 & & $07: 10$ & 900 & o26641 \\
\hline \multirow[t]{2}{*}{184} & A & $05: 17$ & 900 & o26971 \\
\hline & $\mathrm{B}$ & $08: 59$ & 780 & $\mathrm{o} 27061^{c}$ \\
\hline 185 & & 05:49 & 900 & o27341 \\
\hline 186 & & 06:04 & 900 & o27681 \\
\hline \multirow[t]{3}{*}{187} & $\mathrm{~A}$ & 01:44 & 900 & о27961 \\
\hline & B & $06: 12$ & 900 & o28061 \\
\hline & $\mathrm{C}$ & $08: 27$ & 900 & o28121 \\
\hline \multirow[t]{2}{*}{188} & $\mathrm{~A}$ & $05: 33$ & 900 & o28401 \\
\hline & $\mathrm{B}$ & 08:06 & 900 & o28491 \\
\hline \multirow[t]{2}{*}{189} & $\mathrm{~A}$ & $04: 55$ & 900 & o28771 \\
\hline & $\mathrm{B}$ & $08: 20$ & 900 & o28861 \\
\hline 190 & & $05: 53$ & 900 & o29181 \\
\hline 191 & & 05:00 & 900 & o29541 \\
\hline 192 & & 04:34 & 900 & o29941 \\
\hline 193 & & $05: 41$ & 900 & o30401 \\
\hline 194 & & $06: 23$ & 900 & o30731 \\
\hline 195 & & \multicolumn{3}{|c|}{ bad weather } \\
\hline 196 & & $05: 54$ & 1200 & o31151 \\
\hline 197 & & $06: 45$ & 900 & o31561 \\
\hline 198 & & $07: 36$ & 1200 & o31931 \\
\hline 199 & & 05:00 & 900 & o32231 \\
\hline 200 & & 03:40 & 900 & o32591 \\
\hline 201 & & $07: 24$ & 900 & o33051 \\
\hline total & \multicolumn{4}{|c|}{77100} \\
\hline
\end{tabular}

$a$ : Affected by scattered light in the UV-blue region (e.g., $\mathrm{H} \delta, \mathrm{H} \epsilon$, Ca II etc.); $b$ : low signal to noise spectra due to short exposure time; $c$ : strongly contaminated by twilight.

marked in Fig. 1 with a horizontal line. Wavelength intervals up to $40 \AA$ are sometimes contaminated by a single bad column because they are in the same direction as the (bend) echelle orders. For the contaminated regions relative flux uncertainties of the order $5-10 \%$ must be expected which produce in the spectrum spurious humps and wiggles. As the instrument set-up remains fixed the bad CCD columns affect always the same spectral regions.

\subsection{Jet absorptions}

Several strong, blue-sifted jet absorptions are visible in our data due to H I, He I, Na I, Ca II and Fe II (see Fig. 1). The identifications are listed in Table 2 together with equivalent widths $(E W)$ for days 153,144, 191 and 193. Days 153 and 191 represent epochs with relatively narrow jet absorptions. During days 144 and 193 high velocity components are present so that the jet absorptions are extremely broad. A flag in Table 2 indicates whether the lines are saturated and deep or not. Figures 2 and 6 illustrates the huge difference in line strengths and structure. For the measurements of $E W$ the narrow emission lines (and the narrow absorptions for Ca II) have been clipped. Because the continuum normalization is uncertain in the region below $3800 \AA$ no $E W$ are given for H10 and H11.

Particularly strong with $E W \gtrsim 10 \AA$ are the jet absorptions from the Balmer lines and Ca II K. In all these lines, except $\mathrm{H} \alpha$, the residual flux in the broad absorption trough is about 0.1 of the continuum flux or less indicating that the absorptions are at least partly saturated. The broad $\mathrm{H} \alpha$ absorptions are less deep and the equivalent widths smaller than for $\mathrm{H} \beta$ or $\mathrm{H} \gamma$, because the continuum has in the red a strong contribution from the red giant (see also Fig. 4). Considering only the $\mathrm{H} \alpha$ jet absorption in the continuum of the hot component would yield corrected $E W$ for $\mathrm{H} \alpha$, which are about a factor $1.6( \pm 0.1)$ 


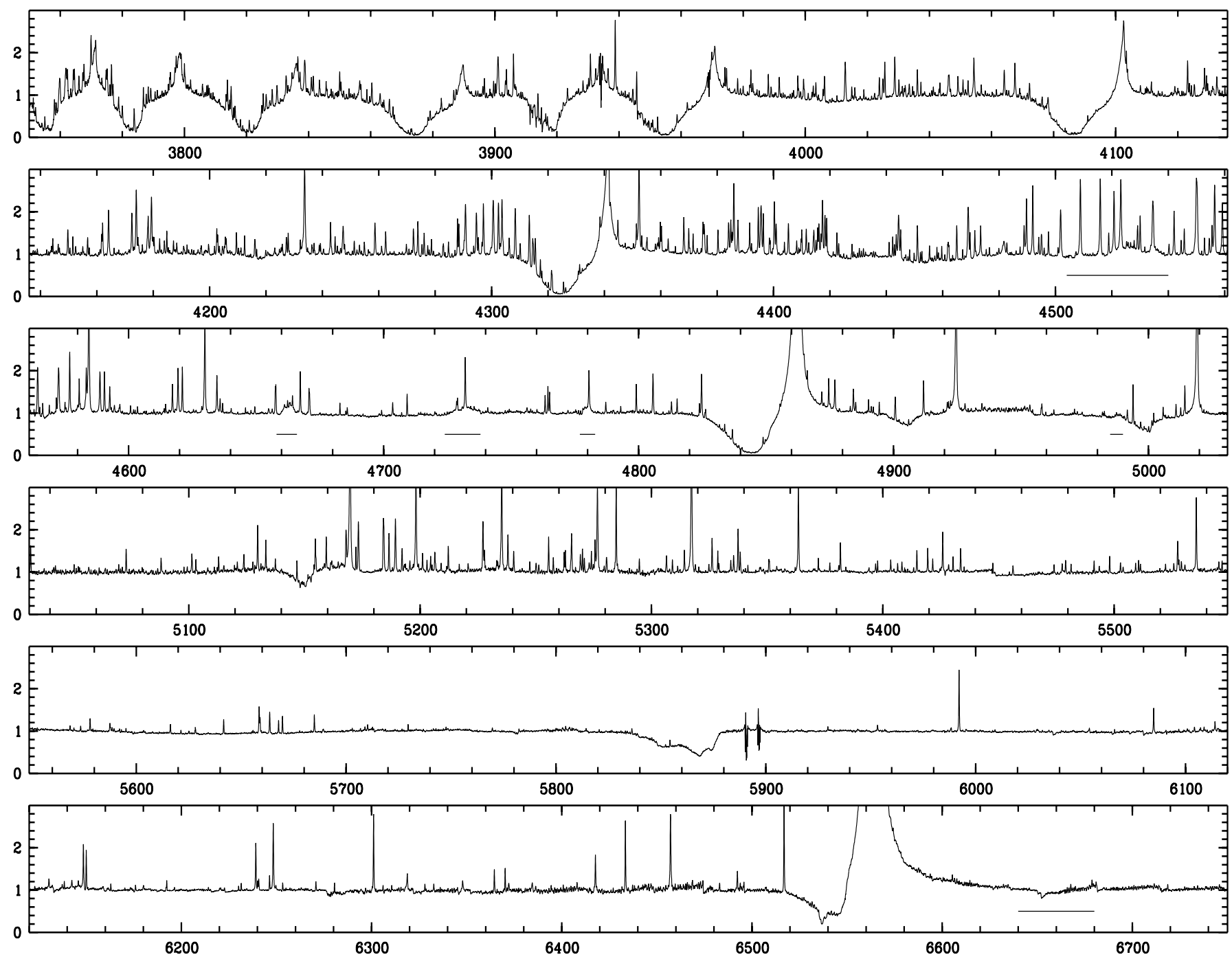

Fig. 1. Spectral atlas (normalized flux) of MWC 560 based on the mean of about 40 high quality spectra. The horizontal lines below the spectrum indicate regions affected by bad CCD pixel columns. The variable, broad jet absorptions appear in this averaged spectrum smooth, although they have for a given observation a very distinctive structure.

larger than the values given in Table 2. $\mathrm{H} \epsilon$ and $\mathrm{H} 8$ are blended with Ca II H and He I $\lambda 3889$, respectively, as can be inferred from the presence of the strong He I $\lambda 5876$ and Ca II K absorptions as well as from the slightly disturbed progression of equivalent widths along the Balmer series.

Of intermediate strengths are the jet absorptions of Fe II multiplet $42 \lambda \lambda 4924,5018,5169$ and the three blended lines from $\mathrm{He}$ I $\lambda 5876, \mathrm{NaI}_{2} \mathrm{D}_{2} \lambda 5890$ and Na I $\mathrm{D}_{1} \lambda 5896$. These absorptions are not saturated. The relative contribution of the individual components in the He I/Na I blend are about 0.45 for He I, 0.35 and 0.20 for $\mathrm{Na} I \mathrm{D}_{2}$ and $\mathrm{D}_{1}$, respectively. Also the Fe II absorption $\lambda 4924$ and $\lambda 5018$ are probably blended with contributions from He I $\lambda 4922$ and $\lambda 5016$, respectively. However, the contribution of He I to these blends is small, at most $30 \%$, as can be estimated from the strengths of the unblended Fe II $\lambda 5169$ and other He I absorptions.

Previous measurements of the $\mathrm{HI}$ jet absorption $E W \mathrm{~s}$ dating from 1990 to 1993 are given in
Tomov \& Kolev (1997). The data taken since the end of 1991 (after the outburst and slow outflow phase) show also rather strong variations $E W \mathrm{~s}$, which stayed, however, always within a well defined range, e.g. $9 \AA<E W(\mathrm{H} \beta)<$ $19 \AA$, similar to our observing period. Also the $E W$ measurements of metal lines from Ca II and Fe II from 1993 (Tomov et al. 1995) are close to our values given in Table 2. Thus, no long term trend in the jet absorption equivalent widths is apparent since Sep. 1991.

Further we have detected many very shallow jet absorptions mainly from HeI and Fe II transitions. Often they are hardly visible in the spectra, which are full of narrow emission lines in the blue and structured by the cool giant absorptions in the red spectral region. However, in the difference spectra of two observations with and without high velocity jet component characteristic features are apparent pointing to the weak jet absorption components. Figure 3 demonstrates this search technique. Measuring the equivalent widths of these weak jet absorptions is very 

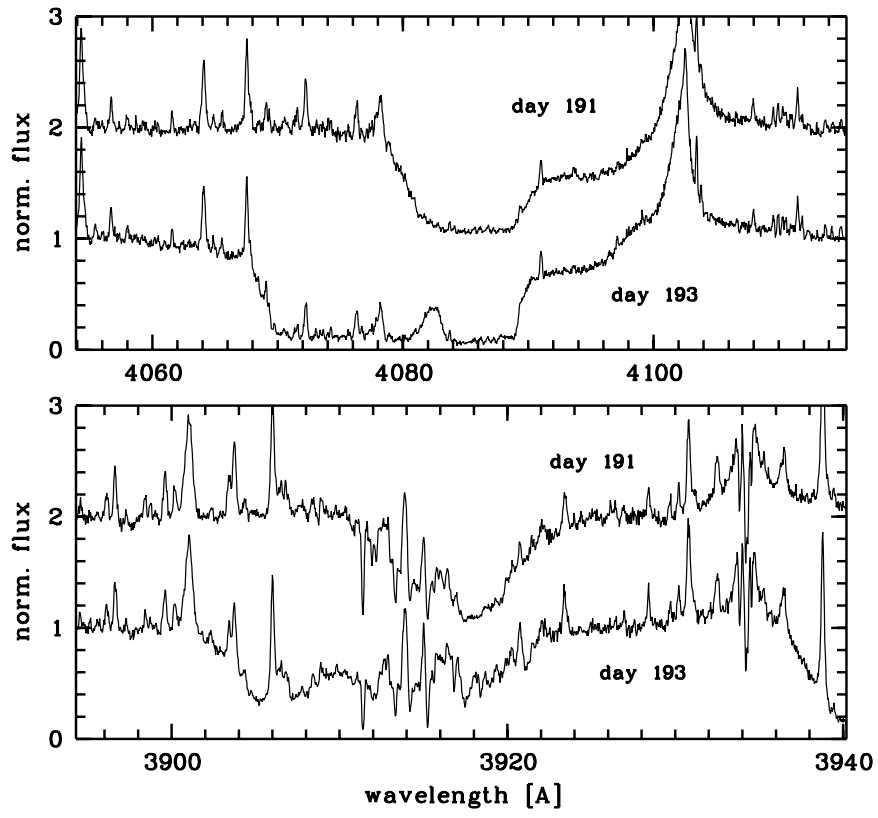

Fig. 2. Spectral changes in the jet absorptions of $\mathrm{H} \delta$ and Ca II K between days 191 and 193. The spectra of day 191 have been shifted for clarity by 1 unit in normalized flux.

Table 2. Equivalent widths $(E W)$ for the strong jet absorptions in MWC 560. Given are values for days 153 and 191 when no high velocity components were present and for days 144 and 193 with strong high velocity components. Estimated measuring errors are $0.5 \AA$, and twice this value for $E W$ s marked with a colon. The column labeled with sat indicates whether the absorptions are saturated.

\begin{tabular}{|c|c|c|c|c|c|c|}
\hline \multirow[t]{2}{*}{ line $\lambda[\AA]$} & \multicolumn{4}{|c|}{$E W[\AA]$} & \multirow[t]{2}{*}{ sat } & \\
\hline & 153 & $144^{a}$ & 191 & 193 & & \\
\hline H11 3770.63 & $b$ & $b$ & $b$ & $b$ & yes & \\
\hline H10 3797.90 & $b$ & $b$ & $b$ & $b$ & yes & \\
\hline H9 3835.39 & 7.8: & 13.2: & 10.4: & 16.2: & yes & $b$ \\
\hline $\left.\begin{array}{l}\text { He I } 3888.65 \\
\text { H8 } 3889.05\end{array}\right\}$ & 10.8 & 15.6 & 12.7 & 20.1 & yes & \\
\hline Ca II K 3933.66 & 6.2 & 8.6 & 5.6 & 8.6 & partly & $c$ \\
\hline $\left.\begin{array}{l}\text { Сa II H } 3968.47 \\
\text { Н } \epsilon 3970.07\end{array}\right\}$ & 11.0 & 16.1 & 12.9 & 19.5 & yes & \\
\hline $\mathrm{H} \delta 4101.74$ & 10.4 & 15.5 & 12.8 & 21.2 & yes & \\
\hline $\mathrm{H} \gamma \quad 4340.47$ & 11.0 & 17.1 & 13.7 & 23.1 & yes & \\
\hline Н г 4471.5 & 2.7 & 2.5 & 2.4 & 2.1 & no & \\
\hline $\mathrm{H} \beta 4861.33$ & 11.7 & 18.9 & 15.2 & 26.0 & yes & \\
\hline Fe II 4923.92 & 2.3 & 2.8 & 2.0 & 2.6 & no & $d$ \\
\hline Fe II 5018.43 & 4.9 & 4.3 & 4.1 & 3.7 & no & $d$ \\
\hline $\begin{array}{l}\text { Fe II } 5169.03 \\
\text { He I } 5875.6\end{array}$ & 3.0 & 4.0 & 2.6 & 2.9 & no & $e$ \\
\hline $\begin{array}{l}\text { Na I } 5889.95 \\
\text { Na I } 5895.92\end{array}$ & 10.4 & 16.1 & 10.0 & 16.7 & no & $e$ \\
\hline $\mathrm{H} \alpha 6562.82$ & 9.9 & 14.2 & 11.0 & 15.8 & yes & $e$ \\
\hline
\end{tabular}

$a$ : Mean of two observations; $b$ : problems with continuum definition; $c$ : includes correction for fast, narrow absorption components; $d$ : blended with He I 4921.93 or 5015.68 respectively; $e$ : red giant contributes significantly ( $\gtrsim 10 \%)$ to the continuum.
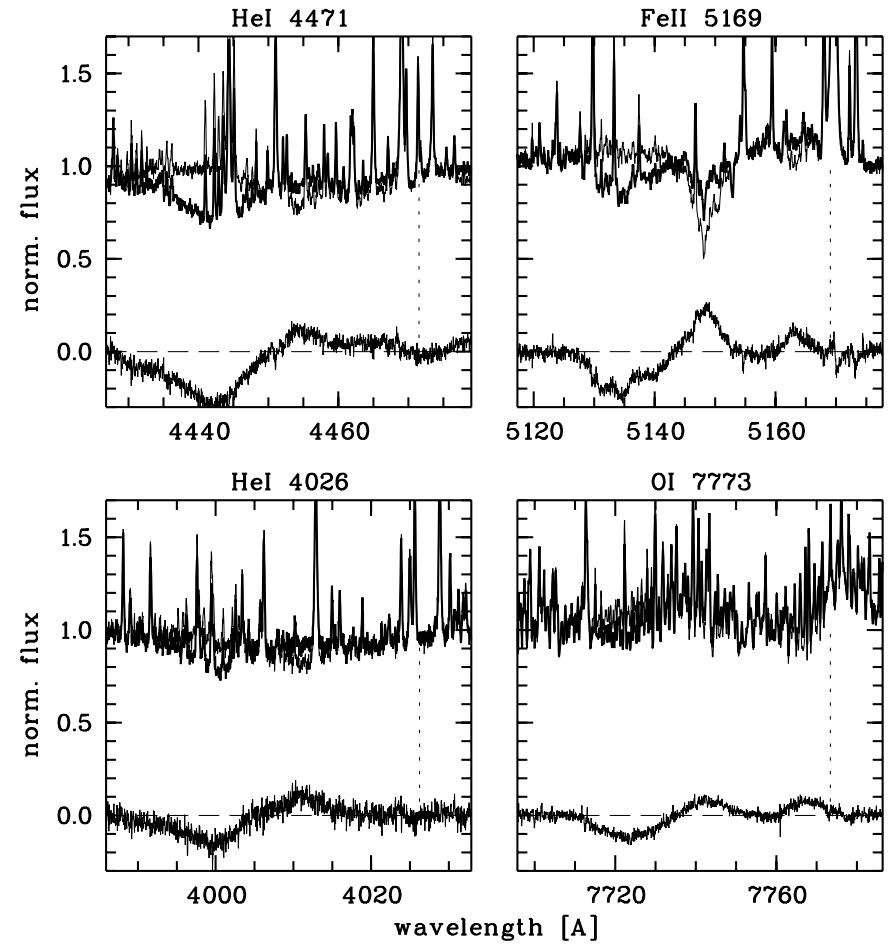

Fig. 3. Identification of weak jet absorptions with the help of difference spectra. The panels show normalized spectra for He I $\lambda 4471$ and $\lambda 4026$, Fe II $\lambda 5169$ and O I $\lambda 7773$ for day 191 and day 193 (with strong high velocity component) and the corresponding difference spectra $F_{193}-F_{191}$. The plotted wavelength ranges corresponds to the $R V$-range -3000 to $+500 \mathrm{~km} \mathrm{~s}^{-1}$ of the considered transition (the dotted line gives $R V=$ $\left.0 \mathrm{kms}^{-1}\right)$.

difficult, because this depends critically on a very accurate continuum definition. We found it more reliable to measure the strengths of the difference features relative to the stronger comparison absorptions He I $\lambda 4471.5$ (for the He I lines) and Fe II $\lambda 5169$ (for the metal lines). Table 3 gives the relative strengths of weak jet absorptions estimated with this procedure. It should be noted that the values correspond to equivalent width ratios relative to the total continuum. Thus the strong contribution of the red giant spectrum must be considered for the interpretation of the strengths of the red jet absorptions, especially He I $\lambda 7065.2$ and O I $\lambda 7773.4$.

Several of the weak jet absorptions are identified here for the first time for MWC 560. Of interest are the detections of weak jet absorptions from Si II multiplet 2 and $\mathrm{O}$ I multiplet 1 , which may be used in connection with UV absorptions for gas temperature determinations. Further jet absorptions are due to He I transitions as expected from the presence of the strong $\lambda 5876$ transition. In addition there are weak absorptions from the most prominent lines of different well known Fe II multiplets. Other Fe II absorptions are present besides those listed in Table 3, but they are either weaker or affected by blending. The Fe II absorptions $\lambda 4233, \lambda 4549$, and $\lambda 4584$ were seen previously by Kolev \& Tomov (1993) during the 1990/1991 low velocity phase. These Fe II lines appeared then together with 
Table 3. Identification of weak jet absorptions. Absorption strengths are given as ratio relative to a strong comparison line based on measurements of the feature strengths in difference spectra (see text and Fig. 3).

\begin{tabular}{|c|c|c|c|c|}
\hline $\begin{array}{l}\text { identification } \\
x\end{array}$ & $\begin{array}{l}\text { comparison line } \\
y\end{array}$ & $\begin{array}{l}\text { ratio } \\
x / y\end{array}$ & $\begin{array}{c}\text { error } \\
\pm\end{array}$ & \\
\hline He I 4026.2 & He I 4471 & 0.48 & 0.15 & \\
\hline Fe II $(27) 4233.17$ & Fe II 5169 & 0.17 & 0.08 & \\
\hline Не г 4387.9 & He I 4471 & 0.15 & 0.08 & \\
\hline Fe II (38) 4549.47 & Fe II 5169 & 0.25 & 0.15 & $a$ \\
\hline Fe II (38) 4583.83 & Fe II 5169 & 0.18 & 0.08 & \\
\hline Fe II (37) 4629.33 & Fe II 5169 & 0.10 & 0.07 & \\
\hline Не г 4713.9 & He I 4471 & 0.11 & 0.07 & \\
\hline Fe II (49) 5316.61 & Fe II 5169 & 0.15 & 0.08 & \\
\hline Si II (2) 6347.09 & Fe II 5169 & 0.08 & 0.06 & $b, c$ \\
\hline Si II (2) 6371.36 & Fe II 5169 & 0.08 & 0.06 & $b, c$ \\
\hline Не г 6678.2 & Не I 4471 & 0.68 & 0.15 & $b$ \\
\hline Не і 7065.2 & He I 4471 & 0.26 & 0.08 & $b$ \\
\hline O I (1) 7773.4 & Fe II 5169 & 0.87 & 0.25 & $b, d$ \\
\hline
\end{tabular}

$a$ : Disturbed by a weaker FeII (37) 4555.89 jet absorption; $b$ : red giant contributes significantly $(>25 \%)$ to the continuum; $c$ : the two Si II lines are seperated by only $R V=1300 \mathrm{~km} \mathrm{~s}^{-1}$, so that the measurement is affected by blending; $d$ : the O I feature is composed of three lines with rest wavelengths at $7771.96,7774.18$ and $7775.40 \AA$.

Ti II absorptions indicating a lower ionization degree of the outflowing gas at that time.

\subsection{Spectral features of the red giant}

Redward of the $\mathrm{H} \beta$ line the contribution of the red giant to the spectrum becomes more and more significant. Strong TiO band heads, e.g., $\lambda 4954, \lambda 5448, \lambda 6681$ and others, can be easlily recognized in the mean spectrum plotted in Fig. 1. The near-infrared portion $\lambda>6750 \AA$ of our FEROS-spectra is dominated by strong $\mathrm{TiO}$ bands. For the interpretation of the jet absorptions depths it is necessary to estimate the strength of the red giant spectrum. For this we compare the observable red giant features in our MWC 560 spectra with a comparison star of similar spectral type. The molecular features in the near infrared indicate a spectral type of M5.5 III for the red giant in MWC 560 (e.g., Meier et al. 1996; Mürset \& Schmid 1999). For our comparison spectrum we have observed with FEROS (on day 145) the red giant S Lep, which is classified as M6+ IIIa by Keenan \& McNeil (1989).

The spectrum of S Lep is shown in Fig. 4 for the $\mathrm{H} \alpha$ and $\mathrm{NaI} / \mathrm{He}$ I region in comparison with the MWC 560 spectrum for day 153 . The S Lep spectrum has thereby been scaled to match the strengths of the red giant features seen in MWC 560 and the flux level obtained for $\mathrm{S}$ Lep yields the contribution of the red giant in the MWC 560 spectrum.

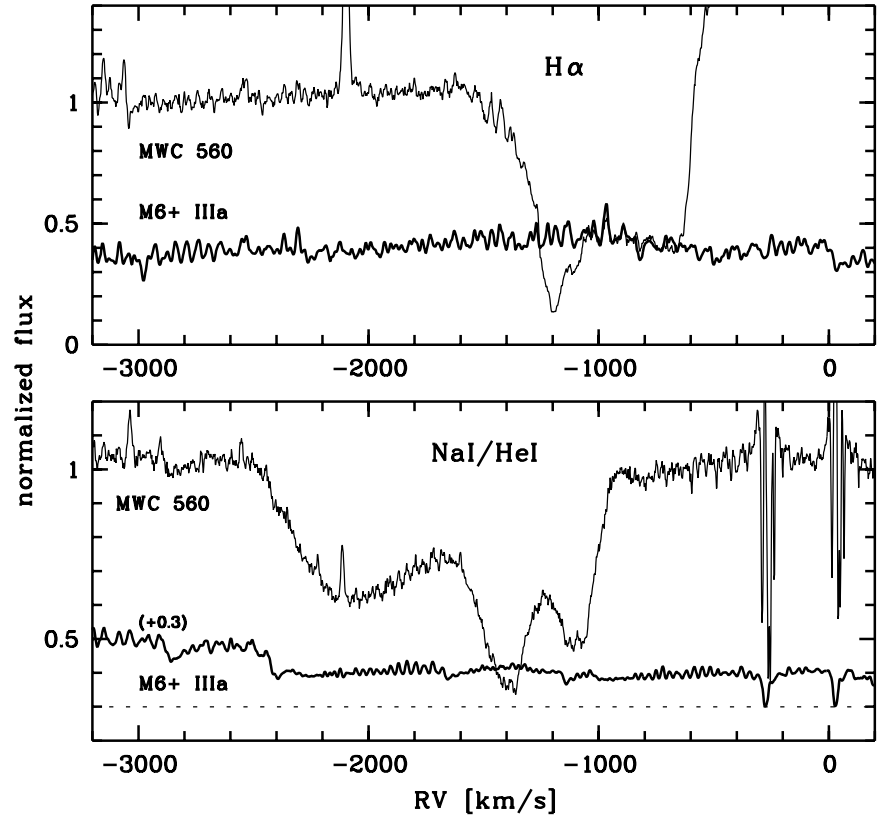

Fig. 4. Estimated contribution of the red giant to the total continuum in MWC 560 in the $\mathrm{H} \alpha$ and the $\mathrm{Na}$ I/He I spectral regions for day 153. The spectrum of the comparison star S Lep (spectral type M6+ IIIa) has been scaled in order to match the strength of the red giant features in MWC 560. The dotted line in the lower panel indicates the zero level for the shifted $(+0.3)$ $\mathrm{S}$ Lep spectrum. Note that the $\mathrm{H} \alpha$ jet absorption obscures at $R V \approx-1200 \mathrm{~km} \mathrm{~s}^{-1}$ a substantial fraction of the red giant spectrum.

In the broad jet absorption trough of the $\mathrm{H} \alpha$ line the red giant spectrum is exposed practically free from contributions of the hot component. This region is therefore ideal for measuring the relative strength of the red giant spectrum. We measure $f_{\mathrm{rg}}$ as mean relative flux in the $R V$-range -1000 to $-850 \mathrm{kms}^{-1}$ corresponding to the wavelength range $6541 \AA$ to $6544 \AA$. The jet absorption for the $\mathrm{H} \alpha$ line is around $R V \approx-1200 \mathrm{~km} \mathrm{~s}^{-1}$ deeper than the estimated continuum of the red giant. This indicates that the jet gas absorbs for this velocity a substantial fraction of the red giant spectrum. In Sect. 5 the properties of this absorption are discussed in more detail.

The flux of the hot component in MWC 560 varies strongly on times scales of hours, as will be discussed in the next section. Therefore, the relative contribution of the red giant to the total continuum varies by about $\sigma=20$ $25 \%$ around a mean value. During our observations the mean contributions of the red giant relative to the total continuum at the position of important jet absorptions are: $3 \%$ for $\mathrm{H} \beta, 6 \%, 3 \%$ and $9 \%$ for Fe II $\lambda 4924, \lambda 5018$ and $\lambda 5169$, respectively, $15 \%$ for He I $\lambda 5876,10 \%$ for Na I $\mathrm{D}_{1,2}$ and $45 \%$ for $\mathrm{H} \alpha$ (see Fig. 4).

\subsection{Variability of the hot continuum}

It is known from various studies that the blue continuum in MWC 560 is strongly variable. The measured variations have an amplitude of the order of 0.1-0.3 mag 

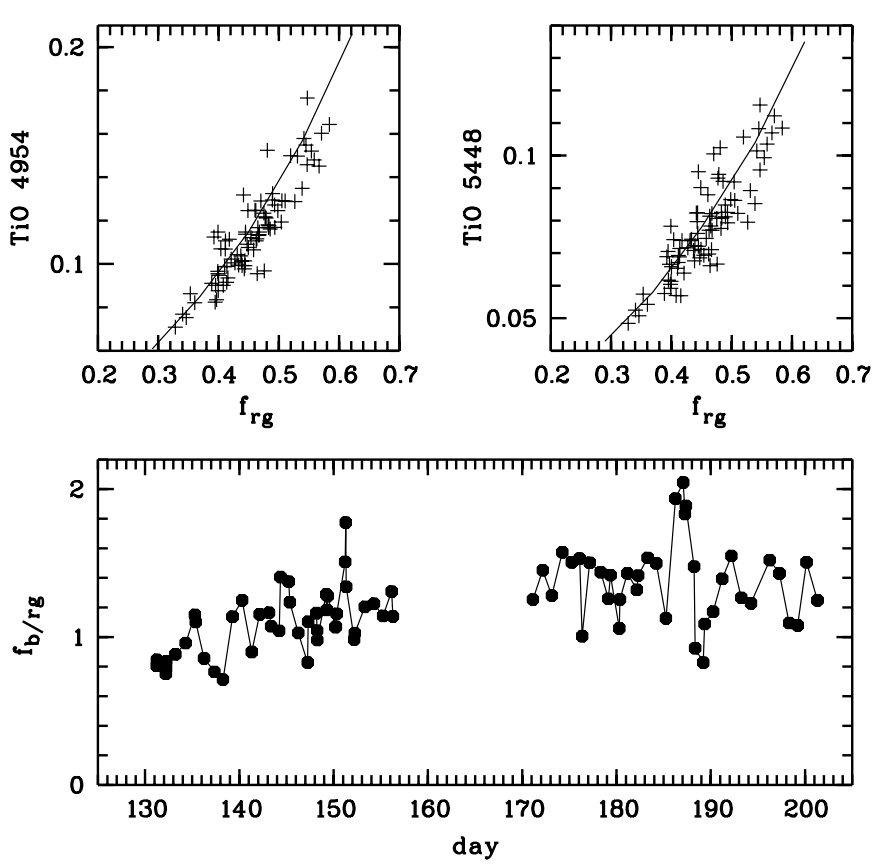

Fig. 5. Relative strengths of red giant features and hot continuum variability. The two upper panels show the correlation of the red giant continuum $f_{\mathrm{rg}}$ measured in the $\mathrm{H} \alpha$ jet absorption and the strengths of the TiO band at $\lambda 4954$ and $\lambda 5448$. The solid curves are lines along which the relative strengths of the red giant features (assumed to be constant on an absolute scale) would move for colour independent flux variations of the hot continuum. The lower panel shows the flux variations for the hot continuum relative to the red giant spectrum. It must be considered that possible variations on time scales of hours are not resolved by the sampling of our data points.

in the $V$-band on a typical time scale of hours (e.g. Michalitsianos et al. 1991; Tomov et al. 1996). Thereby short term brightness changes as large as $\Delta U=0.6 \mathrm{mag}$ have been measured in the $U$-band.

We use the red giant features to measure the relative strength of the hot continuum assuming that the red giant flux is constant. Thus, the hot component is bright when the relative strengths of the $\mathrm{TiO}$ bands are low and the $\mathrm{H} \alpha$ jet absorption is deep (see Fig. 13).

First we check the correlation between the relative flux of the red giant $f_{\mathrm{rg}}$ measured at $6542.5 \AA$ in the $\mathrm{H} \alpha$ jet absorption and the strengths of the $\mathrm{TiO}$ bands $f_{\mathrm{TiO}}$. For $f_{\mathrm{TiO}}=f_{1}-f_{2}$ we measure the difference in flux on the blue $f_{1}$ and the red side $f_{2}$ of the band heads. The mean strengths of the $\mathrm{TiO}$ features is $\left\langle f_{1}-f_{2}\right\rangle=0.115$ for $\mathrm{TiO} \lambda 4954$ and 0.078 for $\mathrm{TiO} \lambda 5448$. The relative scatter for $f_{\mathrm{TiO}}$ is $\sigma=19 \%$ for both the $\lambda 4954$ and $\lambda 5448$ features during our campaign. The two upper panels in Fig. 5 illustrate that the relative strengths of the $\mathrm{TiO}$ bands are well correlated with the relative red giant flux $f_{\text {rg }}$ as measured in the $\mathrm{H} \alpha$ absorption. The solid curves in these panels are lines along which the relative strengths of the red giant features (which are assumed to be constant on an absolute scale) would move if the absolute flux of the hot continuum is varied. The fact that the observed points lie along these lines indicates that the flux variations of the hot continuum are at least to a first approximation colour independent in the $4900 \AA$ to $6600 \AA$ range.

The flux of the hot continuum relative to the cool giant continuum $f_{\mathrm{b} / \mathrm{rg}}$ is given by the re-normalization of the total continuum flux (initially normalized to $f=1$ ) to the red giant continuum $f_{\mathrm{rg}}$ in the $\mathrm{H} \alpha$ jet absorption according to:

$f_{\mathrm{b} / \mathrm{rg}}=\frac{1}{f_{\mathrm{rg}}}-1$.

The lower panel in Fig. 5 illustrates the flux changes of the hot component $f_{\mathrm{b} / \mathrm{rg}}$. The mean is $\left\langle f_{\mathrm{b} / \mathrm{rg}}\right\rangle=1.22$ for the entire campaign. A small long term flux increase can be recognized. The relative flux scatter (deviation) is $\sigma=23 \%$ and extremes are 0.71 and 2.04 for days 138 and 187 , respectively. Variations are sometimes very fast, e.g., for day 151 the relative flux increases from 1.51 to 1.77 within about $80 \mathrm{~min}$ and then drops to 1.34 during the following $80 \mathrm{~min}$. Considering these short term variations it is unclear, whether the flux maximum during days 186 and 187 and the following deep flux drop and minimum for the days 188 and 189 represent a continuous flux variation on a time scale of $\sim$ day, or whether this excursion is a fortuitous feature of strongly under sampled shorter term variations.

The values $f_{\mathrm{b} / \mathrm{rg}}$ are of course only valid for the region around $\lambda 6540$. But as was shown above, the relative flux changes of the hot continuum are at least roughly colour independent and therefore representative for a broad wavelength range.

\section{Jet absorption structure}

The structures of the jet absorption in MWC 560 are strongly variable. The absorptions recorded during our campaign showed a relatively stable component around a radial velocity $\left(R V^{1}\right)$ of roughly $-1100 \pm 200 \mathrm{~km} \mathrm{~s}^{-1}$. In addition there appeared repeatedly highly blue-shifted components with $R V \mathrm{~s}$ up to and beyond $-2000 \mathrm{kms}^{-1}$. Characteristic for MWC 560 is that the jet absorptions are detached from the center of the emission components by about $\Delta R V \approx-600$ to $-1000 \mathrm{~km} \mathrm{~s}^{-1}$. This produces a region without or with only little absorption between the systemic velocity of the line (i.e. the center of the emission component) and the main jet absorption trough.

Figure 6 illustrates the absorption structures for the Hi Balmer lines, HeI, NaI, FeII and CaII for day 153 and day 144. For day 153 the absorptions extend to $-1450 \mathrm{kms}^{-1}$, while for day 144 a high velocity component is present so that the absorptions extend to about $-2150 \mathrm{~km} \mathrm{~s}^{-1}$ (at a residual flux level of 0.8). These two particular dates give a representative comparison between

\footnotetext{
1 Throughout this paper $R V$ s are barycentric $R V$ s unless explicitly indicated. The systemic velocity of the MWC 560 system is $+37( \pm 3) \mathrm{km} \mathrm{s}^{-1}$ and a corresponding correction must be applied for $R V$ s relative to the center of mass of MWC 560 .
} 

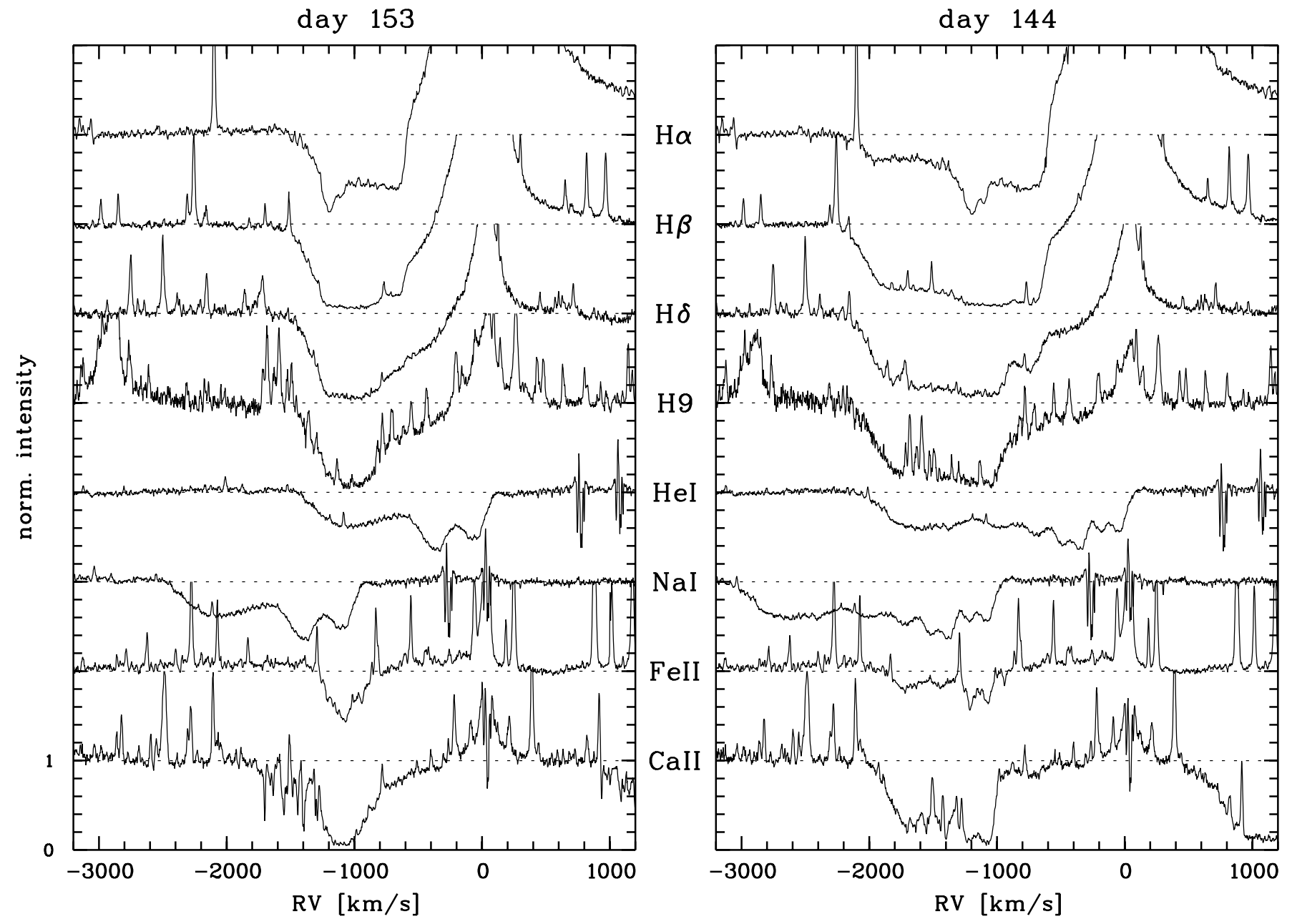

Fig. 6. Comparison of the jet absorption line structure of $\mathrm{H} \alpha, \mathrm{H} \beta, \mathrm{H} \delta$, H9, He I $\lambda 5876$, Na I $\lambda 5896$, Fe II $\lambda 5169$ and Ca II K $\lambda 3934$ for day 153 and day 144. During day 153 no high (negative) velocity component is present while such a component is strong for day 144. The normalized radial velocity (barycentric) spectra are shifted in flux by integer units. The same spectral region is plotted for He I and $\mathrm{Na}$ I but shifted according to the rest wavelengths of the corresponding transitions. Note the blending due to the second line of the Na I resonance doublet at $\lambda 5890$.

times with and without high velocity jet absorption component. During our observing campaign the high velocity components behave for a given day similar for all lines in the optical wavelength region. There are interesting exceptions to this general rule, mainly for the He I lines as will be discussed below. In the following paragraphs we discuss some general features of the jet absorptions.

$\mathrm{H}$ । Balmer lines. All Balmer lines from $\mathrm{H} \beta$ to $\mathrm{H} 11$ produce strong and deep jet absorptions. For $\mathrm{H} \alpha$ the absorption is less deep due to the significant contribution of the red giant spectrum to the total continuum (see Sects. 2.3 and 5).

For $\mathrm{H} \beta, \mathrm{H} \delta$ and $\mathrm{H} 9$ the absorption troughs are over a broad range saturated. Around $R V=-1000 \mathrm{~km} \mathrm{~s}^{-1}$ the residual flux is for all three lines less than $10 \%$ of the continuum flux for day 153, day 144, and also for practically all other days covered by our campaign.

In the high (negative) velocity component of day 144 the residual flux at $-1800 \mathrm{kms}^{-1}$ is about $27 \%$, again practically the same for all three lines $\mathrm{H} \beta, \mathrm{H} \delta$ and $\mathrm{H} 9$.
The oscillator strength $f_{i j}$ and the corresponding line opacity is about a factor of 20 larger for $\mathrm{H} \beta$ than $\mathrm{H} 9$. This requires that the strongly blue-shifted jet absorptions are highly saturated for the Balmer transitions. Thus the high velocity components are opaque in the Balmer lines but they usually do not fully cover the hot continuum source. A further property of the H I absorptions is their smooth structure without narrow subcomponents. Thereby the residual flux is always increasing or at least constant towards higher outflow velocities but never decreasing. This is equivalent to the statement that the covering factor for the higher outflow velocity gas is smaller than or at most equal to the covering factor at lower velocities down to about $R V=-1200 \mathrm{~km} \mathrm{~s}^{-1}$.

At the low (negative) velocity end of the $\mathrm{H}$ I absorption troughs, around $R V \approx-700 \mathrm{~km} \mathrm{~s}^{-1}$, the situation is different. There the absorptions are always weaker for higher Balmer transitions as can also be seen for days 153 and 144 (Fig. 6). This indicates that the Balmer line opacities are for this velocity region not very high. 
The onset of the broad jet absorption trough at about $-600 \mathrm{~km} \mathrm{~s}^{-1}$ absorbs also the extreme blue wing of the strong $\mathrm{H} \alpha$ and $\mathrm{H} \beta$ emission lines. Thus, these extreme line wings, which are attributed to Raman scattered $\operatorname{Ly} \beta$ and $\mathrm{Ly} \gamma$ line wing photons, must originate from behind the absorbing H I gas (see Sect. 4.5).

A shallow absorption component is visible for the higher Balmer transitions between about $R V \approx-600$ to $-200 \mathrm{~km} \mathrm{~s}^{-1}$ corresponding to the region between the broad absorption trough and the emission line peak. Similarly the strong $\mathrm{H} \alpha$ and $\mathrm{H} \beta$ emission seem to have an additional absorption on the blue side indicative of some sort of low velocity outflow. It can again be concluded that this absorption is saturated but covers only a fraction of the continuum source because the strength of the absorption decreases not for higher Balmer lines. This is described in more detail in Sect. 4.5.

He I lines. The He I $\lambda 5876$ line plotted in Fig. 6 is the strongest HeI jet absorption in our spectra. On the low (negative) velocity side HeI is blended with NaI. The He I $\lambda 5876$ jet absorption has always a very smooth structure and is only moderately deep. The mean residual flux for the radial velocity interval -1500 to $-1000 \mathrm{~km} \mathrm{~s}^{-1}$ lies during our campaign between 0.5 and 0.7 of the continuum level (note that 10 to $20 \%$ of the continuum flux are according to Sect. 2.3 due to the red giant). The He I $\lambda 4471$ absorption is less deep indicating that the He I absorptions from the $2 \mathrm{p}^{3} \mathrm{P}^{\mathrm{o}}$ level are not saturated.

If He I high velocity components are present as for day 144, then they extend as far as the corresponding $\mathrm{H} \mathrm{I}$ Balmer absorptions. However the fast absorptions of He I retreat on a shorter time scale after a velocity maximum when compared to the Balmer lines. The He I high velocity absorption is often deeper than the absorption at intermediate velocity unlike the saturated Balmer lines. Metal lines have also such absorption components but they are narrower and more structured than the He I lines. For the interpretation of these differences in spectral structure and temporal variability it must be considered that the He I absorptions originate from much higher excited transitions than e.g. the Balmer lines or the lines from Fe II.

In our spectra no emission line is visible for He I $\lambda 5876$, neither for any other He I line.

Metal lines of Naı, CaII and Fell. The main absorption components $R V \approx-1100 \mathrm{~km} \mathrm{~s}^{-1}$ for the lines of NaI, CaII and FeII coincide in radial velocity with the deep absorptions seen in $\mathrm{H} \mathrm{I}$. Also the extend of the fast absorption component as seen for example during day 144, are comparable to the corresponding feature in $\mathrm{H}$ I. It is the rule that the high velocity components seen in $\mathrm{H}$ I occur simultaneously in the metal lines. There are a few occasions where the high velocity absorptions visible in $\mathrm{H}$ i are too weak to be recognized in the metal lines. For Fe II $\lambda 5196$ for example it is sometimes hard to see a shallow, smooth absorption on top of a spectrum with numerous emission
Table 4. Jet column densities $N_{j}$ calculated from $E W$ and lower limits from $G_{\tau}$-integrals for different atomic species determined from the absorptions of day 144 .

\begin{tabular}{llrlr}
\hline line $(\lambda[\AA])$ & $\begin{array}{c}f_{j k} \\
a\end{array}$ & $\begin{array}{c}E W / G_{\tau} \\
{[\AA]}\end{array}$ & atomic state & \multicolumn{1}{c}{$\begin{array}{c}N_{j} \\
{\left[\mathrm{~cm}^{-2}\right]}\end{array}$} \\
\hline H $\beta$ 4861 & 0.119 & 37.8 & H I, $n=2$ & $>1.5 \times 10^{15}$ \\
H9 3835 & 0.0054 & 26.4 & H I, $n=2$ & $>3.7 \times 10^{16}$ \\
He I 4471 & 0.125 & 2.5 & He I, $2{ }^{3} \mathrm{P}^{\mathrm{o}}$ & $1.1 \times 10^{14}$ \\
Na I 5896 & 0.327 & $3.2^{b}$ & Na I & $3.2 \times 10^{13}$ \\
Ca II 3934 & 0.69 & 14.6 & Ca II & $>1.6 \times 10^{14}$ \\
Fe II 5169 & 0.0057 & 4.0 & Fe II, a ${ }^{6} \mathrm{~S}$ & $3.0 \times 10^{15}$ \\
\hline
\end{tabular}

$a$ : Oscillator strengths are from the compilations of Wiese et al. $(1966,1969)$ and Fuhr et al. (1981); $b$ : assuming that the line absorption of $\lambda 5896$ is $20 \%$ of the HeI/Na I blend.

lines and the (diluted) absorption structure of the red giant.

A major difference to $\mathrm{HI}$ is that the jet absorptions for Fe II and NaI are not saturated. In the case of the Ca II K line saturation occurs only for a relatively narrow $R V$-region. Thus we can see substructures in the main absorption components which are not visible in the saturated $\mathrm{HI}$ lines. The subcomponents occur in the different metal lines simultaneously (see e.g., day 144 in Fig. 6).

A further characteristic of the metal lines is that there is almost no absorption visible for $R V \gtrsim-1000 \mathrm{~km} \mathrm{~s}^{-1}$. This is especially true for the neutral (zero excitation) Na I $\lambda 5896$ absorption which has a well defined low (negative) velocity edge. This indicates that lower velocity gas has an ionization degree which is too high to produce Na I absorptions.

The metal lines producing jet absorptions have also emission components at the systemic velocity. The emission lines structures of the resonance transitions of $\mathrm{Na}$ I and CaII are strongly affected by interstellar absorption components. Further we see for Ca II several blue-shifted, narrow absorption components in the range $R V=-1800$ to $-1200 \mathrm{~km} \mathrm{~s}^{-1}$. Their high velocity indicates a tight connection with the jet outflow. It will be suggested in Sect. 7 that they are produced by shocks in the jet.

\subsection{Column densities}

Column densities for weak (unsaturated) absorptions are proportional to the measured $E W$ (in $\AA$ ) according to the formula

$N_{j}=\frac{1.13 \times 10^{20}}{\lambda^{2} f_{j k}} E W$

Thereby $N_{j}$ is the column density (in units of $\left[\mathrm{cm}^{-2}\right]$ ) of an atom in the lower level $j$ of the transitions $j \rightarrow k$ with wavelength $\lambda$ given in $\AA$ and the oscillator strength $f_{j k}$. This formula can be applied for e.g., the HeI, FeII and Na I jet absorptions in MWC 560. Thereby it is assumed that the absorbing gas is smoothly distributed in front of the continuum source and not in small opaque clumps. 
For saturated jet absorptions accurate column densities are difficult to determine, but lower limits can be derived. For deep absorptions we have to replace in the above formula the equivalent width $E W$ by the wavelength integral $G_{\tau}$ (measured in $\AA$ ) of the jet gas optical depth $\tau(\lambda)=-\ln I_{\lambda}$, where $I_{\lambda}$ is the (residual) intensity of the jet absorption in the normalized spectrum:

$G_{\tau}=\int-\ln I_{\lambda} \mathrm{d} \lambda$

This procedure for measuring column densities is discussed in e.g. Korista et al. (1992) for the absorptions in BAL-quasars. The treatment assumes that: (i) the jet gas distribution is homogeneous and completely covers the continuum source; (ii) the emission from the jet gas is negligible; and (iii) that there is no scattered light. Point (i) is certainly violated for the jet absorption structures in MWC 560 described above. Further we have to expect scattered light from the spectrograph on the $1 \%$ continuum level. If any of the above assumptions is not fulfilled then the derived optical depths or column density will be an underestimate of the true value. Therefore, we consider all column densities determined from strong lines as lower limits.

The integral $G_{\tau}$ is for the hydrogen ( $\mathrm{H} \beta$ to $\left.\mathrm{H} 9\right)$ jet absorptions $2.0( \pm 0.1)$ times larger than the $E W$ s given in Table 2. The corresponding factor for Ca II K is $1.7( \pm 0.2)$. Derived values and limits for column densities are given in Table 4 for different species based on the absorptions measured on day 144.

The large difference in the lower limit of $N(\mathrm{H} \mathrm{I}, n=2)$ derived from $\mathrm{H} \beta$ and $\mathrm{H} 9$ demonstrates well the difficulty in determining column densities from saturated jet absorptions. In any case, less saturated transitions give more accurate results.

For the total jet column density of hydrogen and helium a population for the highly excited levels $\mathrm{HI}, n=2$ and He I, $2 \mathrm{p}^{3} \mathrm{P}^{\mathrm{o}}$, respectively, has to be adopted. But without detailed knowledge on gas temperature, density, ionization degree, and radiation field these level populations are highly uncertain.

Better suited for an estimate of the total column density of the jet gas are the transitions of the metal lines. The $\mathrm{NaI}$ and Ca II resonance lines yield directly the column density of the corresponding atoms. The Fe II Boltzmann excitation ratio $N\left(\mathrm{a}^{6} \mathrm{~S}\right) / N(\mathrm{Fe}$ II $)$ is about $10^{-3}$ for the electron temperature $T_{\mathrm{e}}=7500 \mathrm{~K}$ and $10^{-2}$ for $15000 \mathrm{~K}$. Thus, the total abundance of singly ionized iron $N$ (Fe II) is more than about $10^{3}$ times higher than $\mathrm{Na}$ I or Ca II. This indicates that sodium and calcium are predominantly in the ionization state NaII and CaIII. This is well possible when comparing the ionization potential of $16.2 \mathrm{eV}$ for Fe II to only $11.9 \mathrm{eV}$ for Ca II or $5.1 \mathrm{eV}$ for Na I.

We conclude that the column density of Fe II in the jet is $N(\mathrm{Fe} I \mathrm{I}) \approx 10^{18 \pm 0.5} \mathrm{~cm}^{-2}$. Assuming further that $\mathrm{Fe} I \mathrm{I}$ is the dominant ionization state of iron and that the iron abundance is solar $N(\mathrm{Fe}) / N(\mathrm{H})=10^{-5.2}$ in
MWC 560 yields a total hydrogen column density of the order $N(\mathrm{H}) \approx 10^{23.2 \pm 0.5} \mathrm{~cm}^{-2}$.

\section{Temporal changes in the jet absorptions}

The variability in the broad jet absorptions is one of the characteristics of MWC 560. Whenever repeated spectral observations were taken it was recognized that the jet absorptions are strongly variable on times scales as short as days or even a few hours (e.g., Bond et al. 1984; Tomov et al. 1990; Michalitsianos et al. 1991; Buckley 1992; Tomov \& Kolev 1997). However, our FEROS high resolution spectra are the first data set where the spectral changes of the jet absorptions are followed during (many) consecutive nights. Therefore a detailed description of the observed changes is given here.

Figure 7 gives a first impression on the observed variations from the evolution of the spectra of the $\mathrm{H} \delta$ and Ca II jet absorptions over eight consecutive nights. The plotted data series includes the appearence and disappearence of a strong high velocity component with $R V$ up to $-2500 \mathrm{~km} \mathrm{~s}^{-1}$ around day 181 which is seen in both the $\mathrm{H} \delta$ and the Ca II line simultaneously.

At the same time the flux between the absorption trough and the emission lines $R V \approx-900$ to $-400 \mathrm{~km} \mathrm{~s}^{-1}$ has a maximum for $\mathrm{H} \delta$. The same is probably true for CaII, but the effect is less pronounced and the continuum determination is less accurate in the crowded UV region. A splitting of the CaII absorption into two components occurs on day 180 and a flux maximum in the middle at $R V=-1300 \mathrm{~km} \mathrm{~s}^{-1}$ appears for three days. Further there seems to be a connection between a subtle shift of the low (negative) velocity edge of the broad absorptions at $-1000 \mathrm{~km} \mathrm{~s}^{-1}$ and the occurrence of the high veloctiy component.

This description and its visualization in Fig. 7 of the complex line variability pattern covers only two lines and a small fraction of our campaign. Due to the lack of space it is not possible to present all the observed spectral variations in such a form. We therefore use other ways to present our data and select those features which seem to be the most important.

\subsection{Dynamical spectra}

An overview on the temporal variations of the jet absorptions can be gained from the dynamical spectra for $\mathrm{H} \beta$, CaII K, the HeI/NaI-blend and FeII $\lambda 5169$ plotted in Fig. 8. The panels show for these lines the normalized radial velocity spectra for the 56 nights covered by our campaign. If more than one spectrum was taken during a given night the exposure time weighted mean was used. All spectra were taken during the same hours of the observing nights (between about UT 02:00 and 09:00) so that an equal spacing between consecutive nights is warranted for the discussion of the day to day evolution. 
HI 4101.73

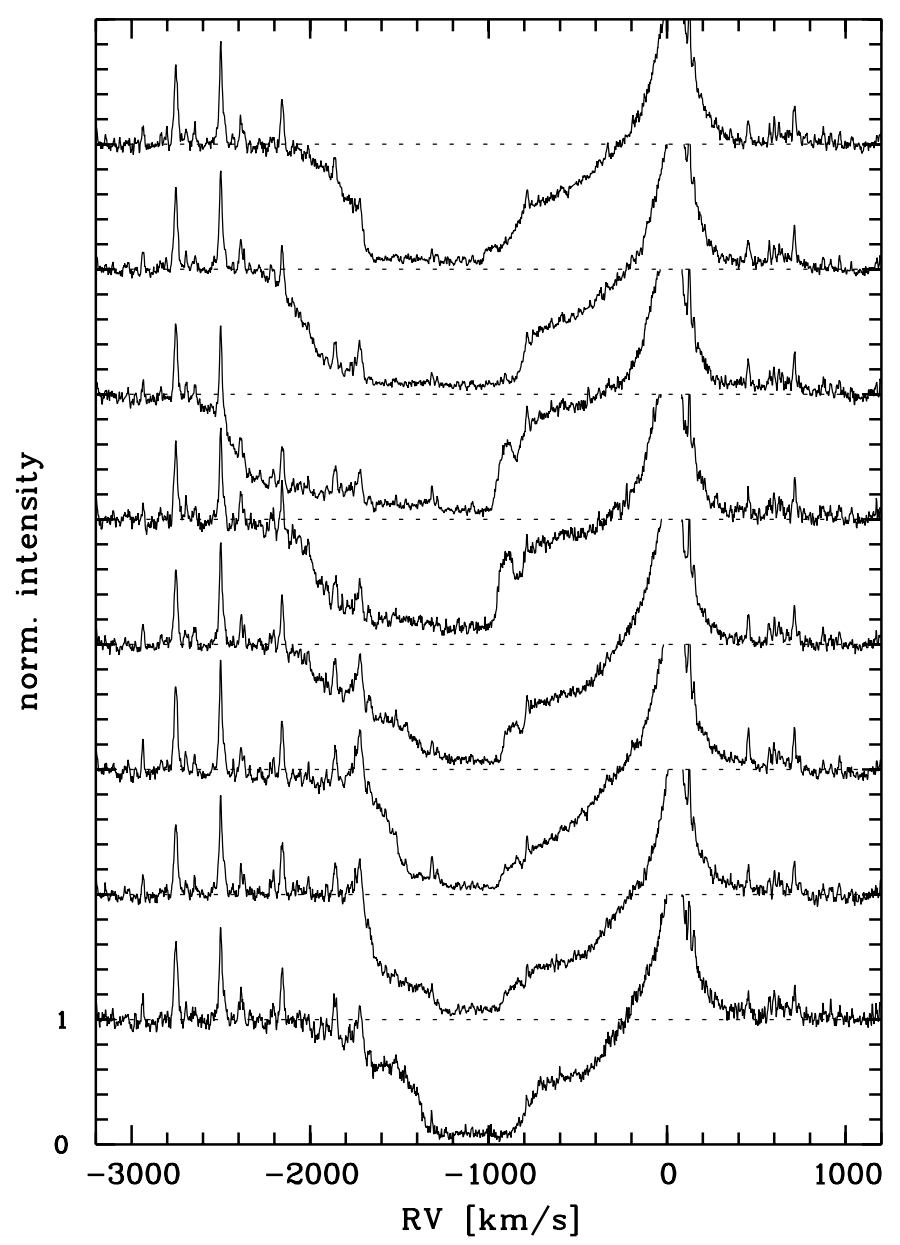

CaII K 3933.66

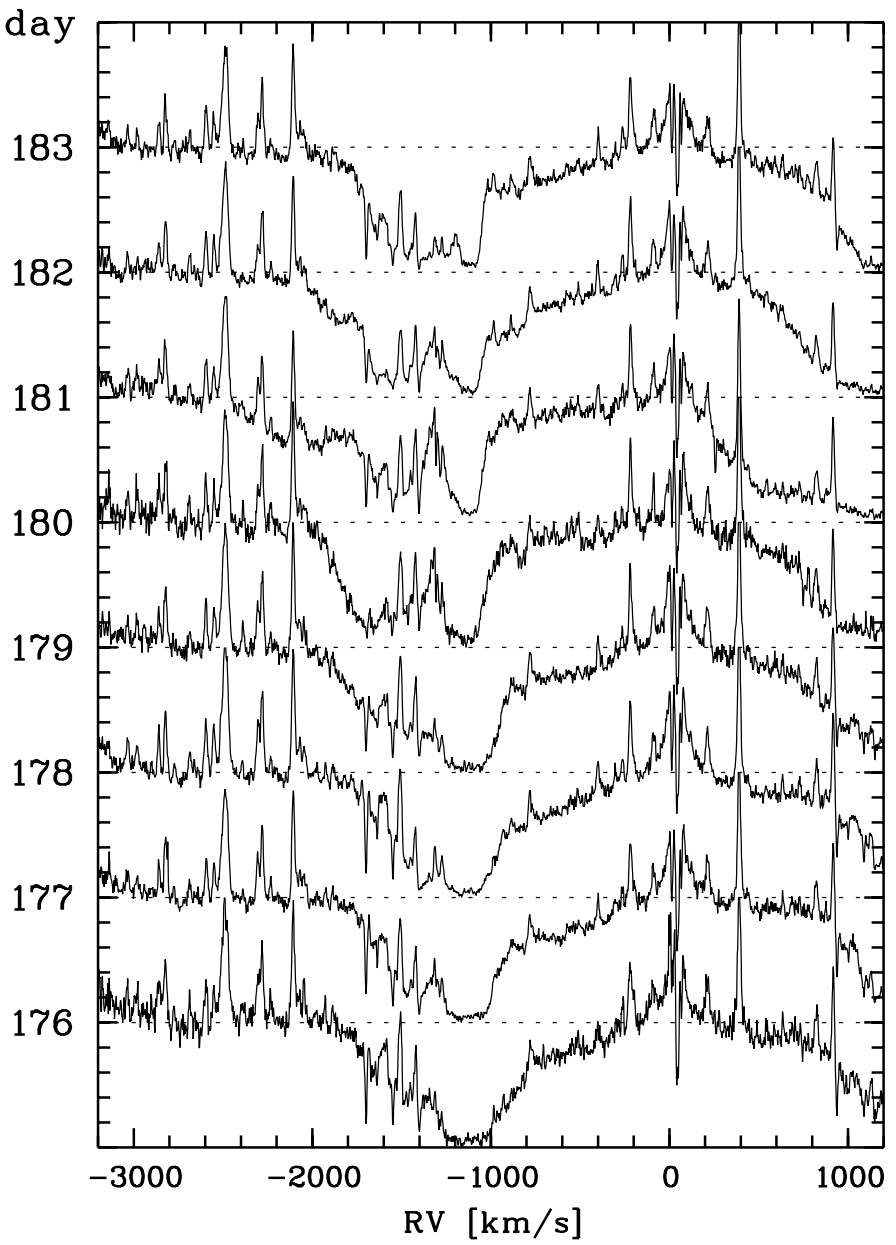

Fig. 7. Temporal variations for the jet absorption structure of $\mathrm{H} \delta$ and CaII K. The sequence shows spectra from days before and after the appearence of the strong high velocity component of day 181. At the positive velocity end of the Ca II K spectra, parts of the high velocity components of the $\mathrm{H} \epsilon / \mathrm{Ca}$ II $\mathrm{H}$ blend are visible.

High velocity components. From Fig. 8 it is apparent that there appear repeatedly high (negative) velocity components, which extend far more to the blue than the "average" absorption through. These transient high velocity components are best seen in the $\mathrm{H}$ I lines (i.e. $\mathrm{H} \beta$ in Fig. 8). There the absorptions reach the maximum blueward-extend within one or two days and then retreat again over the following few days. Each of the transient high velocity components seen in the $\mathrm{H} \beta$ and the other $\mathrm{H}$ I lines are also clearly visible for the He I transitions. Also the CaII and Fe II lines react in step with the other lines, but sometimes the high velocity component is rather weak and therefore only barely visible.

An interesting difference between $\mathrm{HI}$ and $\mathrm{He} \mathrm{I}$ is that the transient high velocity absorption for HeI disappears faster after having reached its maximum strengths. Further the transient high velocity components in He I are relatively strong compared to the absorption around $R V=-1200 \pm 300 \mathrm{~km} \mathrm{~s}^{-1}$. Therefore, the He I $\lambda 5876$ high velocity components are easily seen even if their counterpart in the hydrogen absorptions is relatively weak. Due to the higher excitation potential of the He I transitions, the He I absorption behaviour can be associated with the hotter or higher ionized gas component in the jet outflow.

Main absorption trough. The central region $R V=-1500$ to $-900 \mathrm{~km} \mathrm{~s}^{-1}$ of the jet absorptions is saturated over a broad range for the H I Balmer lines. Variations within this range must therefore be investigated using the weaker absorptions of the metal lines CaII, FeII or NaI and HeI. The center of the main absorption in CaII and Fe II moves in radial velocity back and forth by about $100-200 \mathrm{~km} \mathrm{~s}^{-1}$ around an "average" $R V$. In addition some larger excursions occur which are associated with the appearance of the high velocity components discussed above. For example during the presence of the very strong high velocity component of day 193 the low velocity jet absorptions disappear almost entirely for Fe II and Na I. For He I the $R V$-jitter is even more pronounced. It can be strongly suspected from Fig. 8 that there exists an anti-correlation between the absorption at $-1100 \mathrm{~km} \mathrm{~s}^{-1}$ 
(a) $\mathrm{H} \beta \lambda 4861$

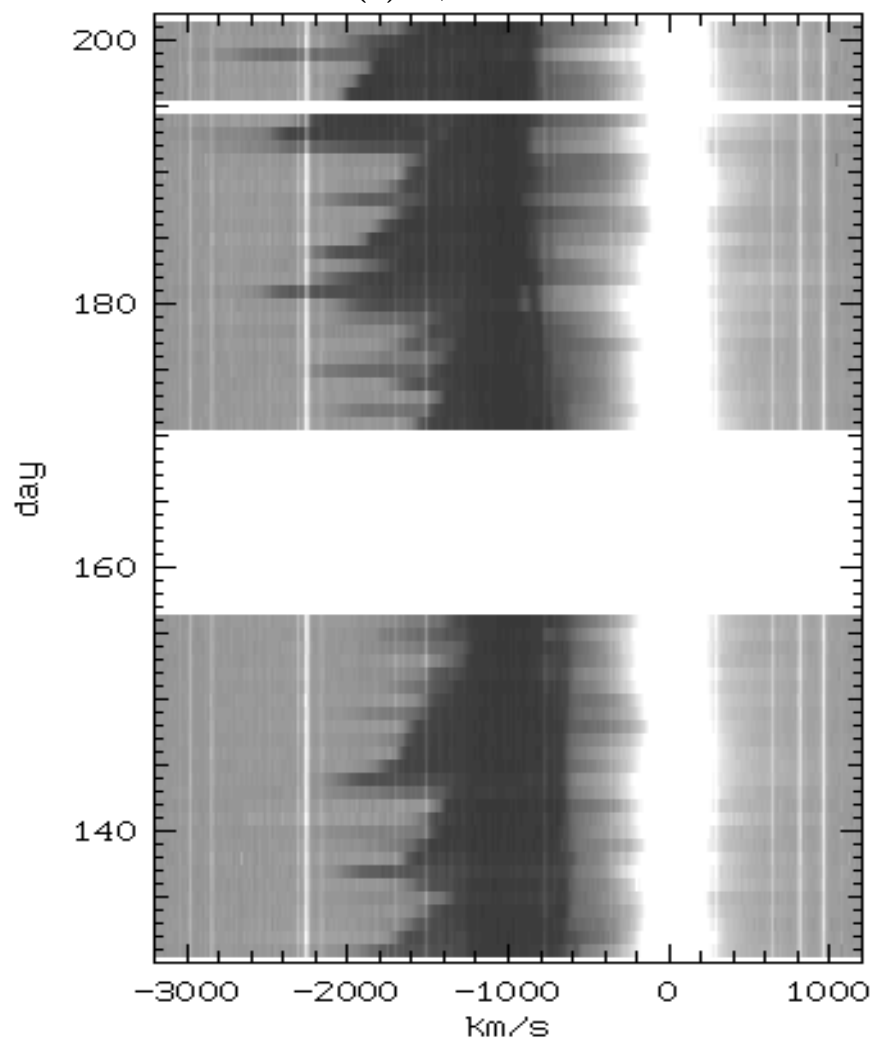

(c) HeI $\lambda \mathbf{5 8 7 6}$ (and $\left.\mathrm{NaI} \mathrm{D}_{2}, \mathrm{D}_{1}\right)$

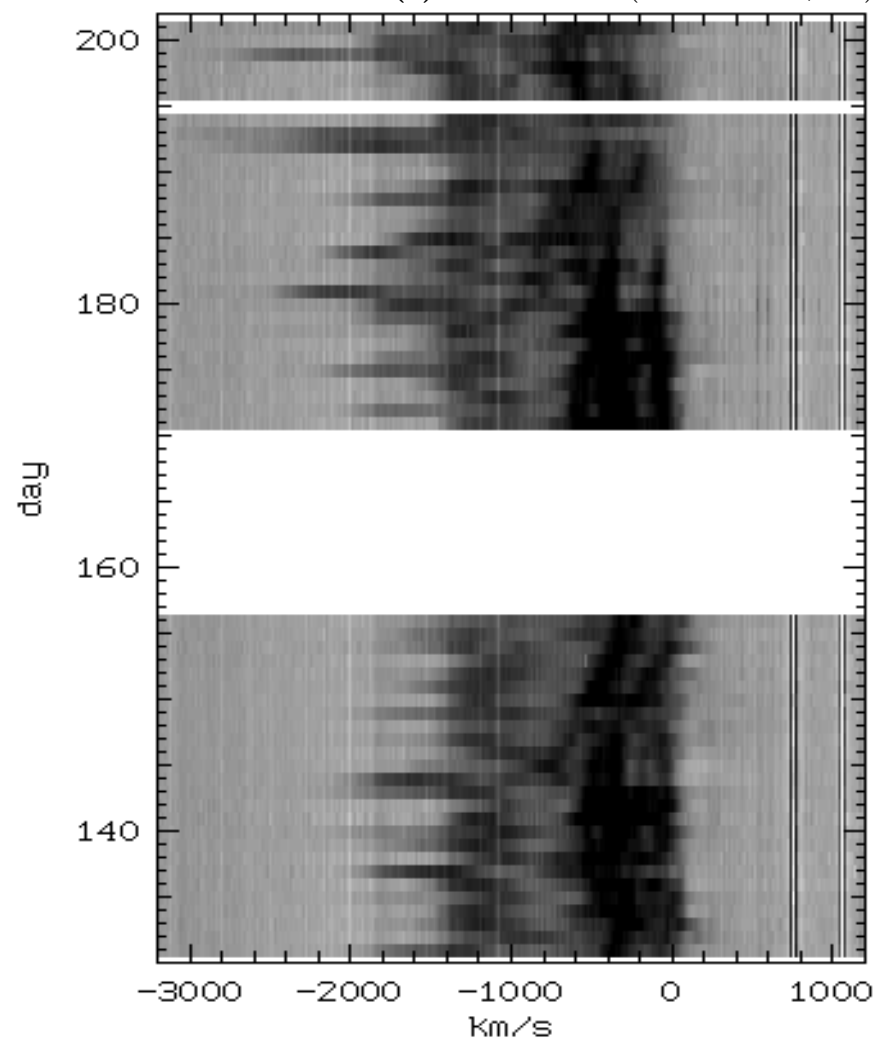

(b) Ca II K $\lambda 3934$

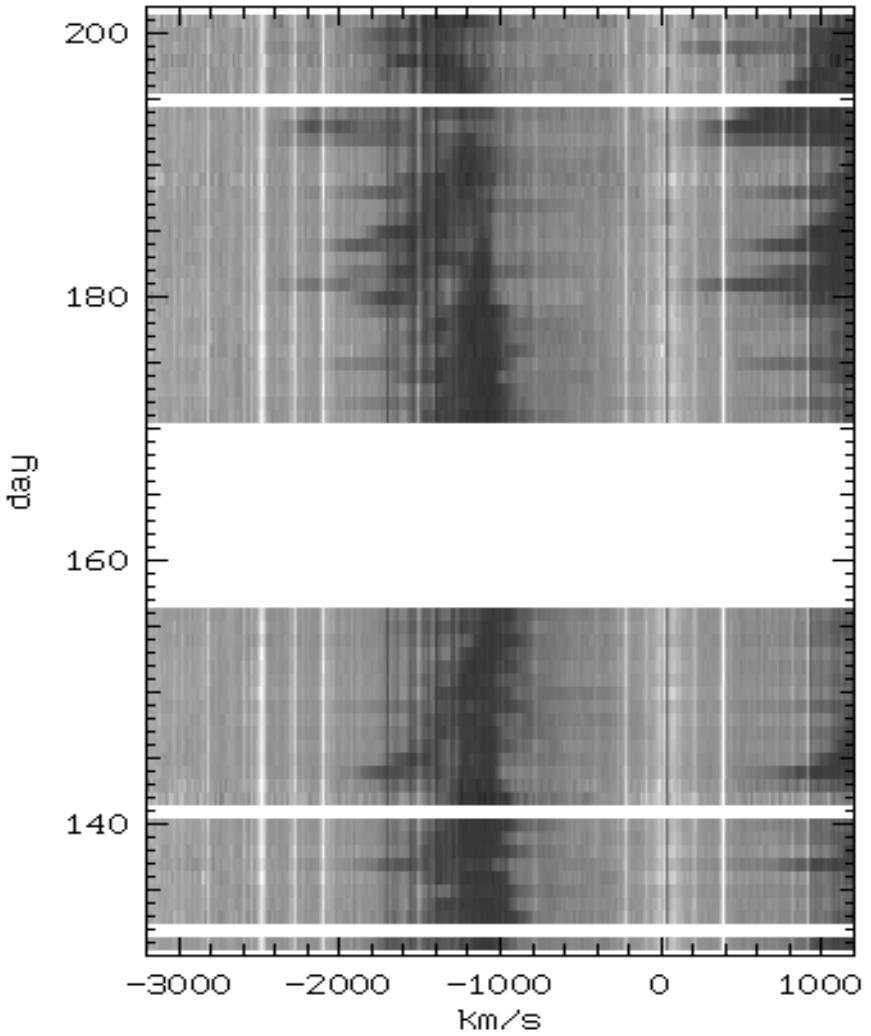

(d) Fe II 5169

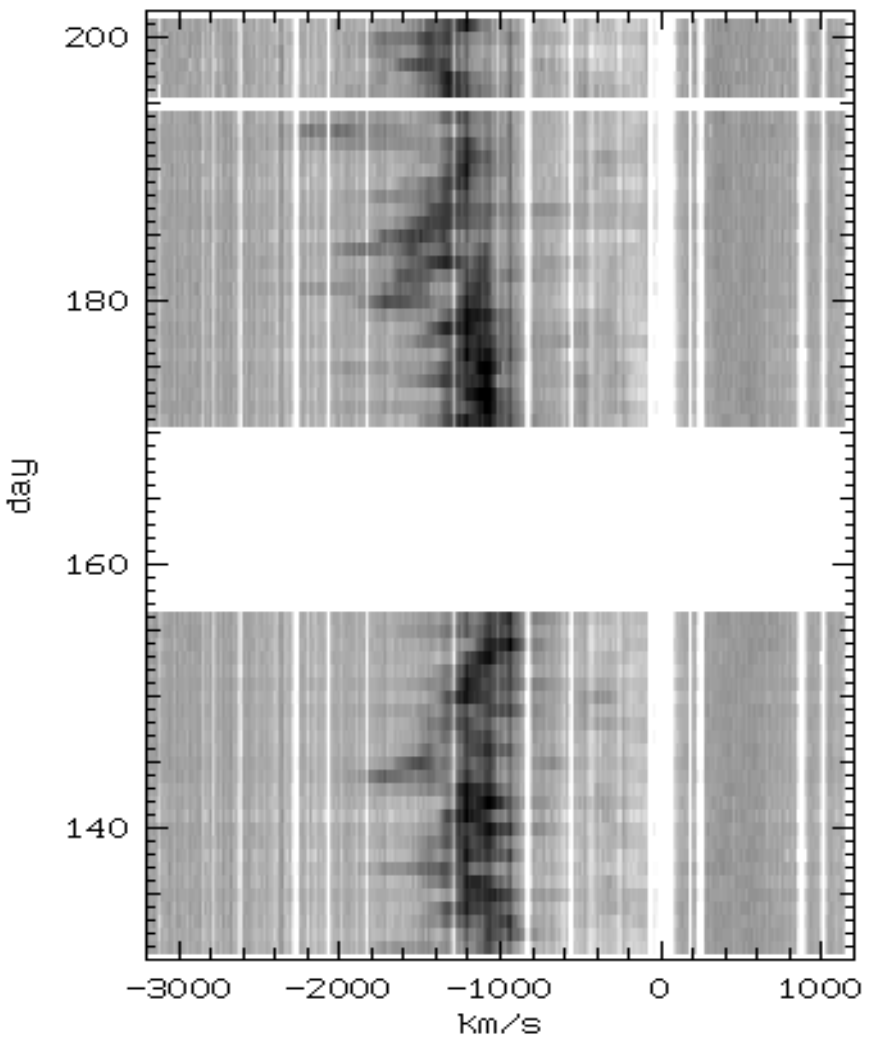

Fig. 8. Dynamical spectra of a) H $\beta$, b) Ca II K, c) He I and d) Fe II for the entire FEROS campaign. The grey scale is linear and black corresponds to low intensity and white to high intensity. The grey scale range is set from -0.5 to 2.0 for the upper panels $\mathbf{a}$ ) and $\mathbf{b}$ ), and from 0.4 to 1.4 for the lower panels c) and $\mathbf{d}$ ). The He I lines is mainly in the region from $R V \approx 0$ to $-1000 \mathrm{~km} \mathrm{~s}^{-1}$ blended by the Na I doublet. 
and e.g., $-1500 \mathrm{~km} \mathrm{~s}^{-1}$ for the metal lines and He I. This indicates that there exists a strong link between the absorption component around $-1100 \mathrm{~km} \mathrm{~s}^{-1}$ and the transient high velocity component.

The radial velocity of the deepest absorption component in CaII and Fe II lines is about $R V=-1100 \mathrm{~km} \mathrm{~s}^{-1}$ for days 131 to 165 . For days 171 to 201 this absorption shifts slowly from $R V=-1100$ to $-1300 \mathrm{~km} \mathrm{~s}^{-1}$. Very much the same long term trend can be recognized for $\mathrm{Na}$ I doublet despite the blending of the two components.

Low velocity absorption. Much less variations are seen at the low (negative) velocity side of the jet absorption troughs. In the $\mathrm{H} \beta$ line the low velocity edge of the trough is at about $-700 \mathrm{~km} \mathrm{~s}^{-1}$ for the first data series from day 131 to 156 . During the second series a slight progression towards $-900 \mathrm{~km} \mathrm{~s}^{-1}$ is visible in step with the progression of the deepest absorption of the metal lines. A similar behaviour, although a bit less well defined, is visible for the low velocity absorption edges of $\mathrm{Na}$ I and Ca II. However for these transitions, the low velocity edges are further to the blue at about $-1000 \mathrm{~km} \mathrm{~s}^{-1}$. The distinct difference between $\mathrm{HI}$ and the metal lines in the $R V$ for the low velocity absorption edge points again to an ionization stratification in the gas outflow.

Very interesting is the behaviour of the shallow low velocity absorption $R V \approx-700$ to $-200 \mathrm{~km} \mathrm{~s}^{-1}$ in $\mathrm{H} \beta$ and the other Balmer lines. The absorption in this low velocity region is very weak if the high velocity component is strong and much more pronounced if the high velocity component is absent. Thus there exists an anti-correlation between the low and high velocity regimes of the absorbing gas, similar to the case of He I and the metal lines described above. In the following sections, this anti-correlation will be investigated in more detail.

\subsection{Differences from day to day}

A very illustrative way to show the fast spectral changes are difference spectra between consecutive observations, which corresponds to a discrete time derivative of the spectral flux. Our data sampling is best suited for plotting the difference between the mean spectrum of consecutive days $\Delta f_{\lambda}=f_{\lambda}\left(\right.$ day $\left._{n+1}\right)-f_{\lambda}\left(\right.$ day $\left._{n}\right)$.

The resulting 2-dimensional daily difference spectrum for $\mathrm{H} \beta$ is plotted in Fig. 9. From this figure it is visible that changes occur mainly for the $R V$-regions -2500 to $-1500 \mathrm{~km} \mathrm{~s}^{-1}$ and -800 to $300 \mathrm{~km} \mathrm{~s}^{-1}$. Between these two regions is the saturated jet absorption trough where the absolute flux changes are small.

The two main variability pattern emerge clearly in Fig. 9.

First, there are the strong changes in the widths of the saturated jet absorption trough, which widens abruptly on the blue side together with the appearence of a strong high velocity component (black in Fig. 9) and then retreats continously towards the inital widths within

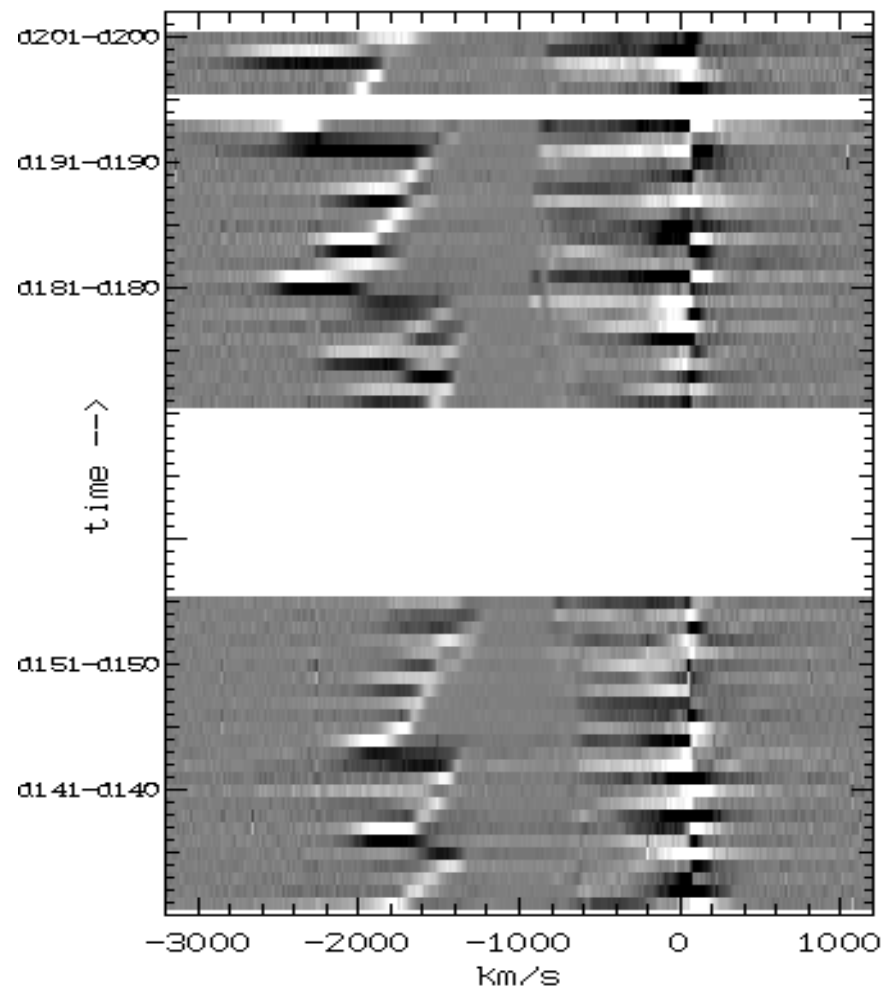

Fig. 9. Daily difference spectra for the $\mathrm{H} \beta$-region. Each grey scale spectrum gives the difference between consecutive nights $\Delta f=f_{\lambda}\left(\right.$ day $\left._{n+1}\right)-f_{\lambda}\left(\right.$ day $\left._{n}\right)$. Black and white indicate a strong decrease or increase, respectively, in normalized flux within one day. Cut values are from $\Delta f=-0.5$ (black) to +0.5 (white). This representation illustrates the widths changes of the jet absorption trough and the faster up and downs on the low $\sim-800$ to $-200 \mathrm{~km} \mathrm{~s}^{-1}$ and high velocity $\sim-2800$ to $\sim-1400 \mathrm{~km} \mathrm{~s}^{-1}$ side of the trough which are anti-correlated.

several days. Such deep absorption components appear on days 137, 143/144, 181 and 192/193 and will be called hereafter major high velocity absorption components.

Second, there are the fast variations or strong daily flux differences on both sides of the absorption trough, which are anti-correlated between the low and high velocity side. This phenomenon is always occurring when a major high velocity absorption component appears. In addition there are also minor high velocity absorption components which are less deep and have a life time of only about $\sim 1$ day. They are best visible in the dynamical spectrum of the He I line in Fig. 8, e.g., for the stronger events occuring for days 184,188 or 199 or weaker events for days $140,172,175$ and probably also for day 149 . The stronger events share the anti-correlation between high and low radial velocity absorption dephts of the major components as is clearly visible in the difference spectra (Fig. 9).

For the weaker short term events the situation is less clear. Because of the limited sampling we may have missed the phase where the minor high velocity component was strong enough for recognizing the anti-correlation phenomenon. Thus we see at least that short lived (or minor) 
high velocity absorptions occur repeatedly on a recurrence time scale of a few days.

The fast variations, i.e. the daily flux differences, extend all the way to the $\mathrm{H} \beta$ emission peak at $R V \approx$ $50 \mathrm{~km} \mathrm{~s}^{-1}$ (see Sect. 4.5). Thus the variation seen around $-100 \mathrm{~km} \mathrm{~s}^{-1}$, due mainly to the varying absorption in the blue emission line wing of $\mathrm{H} \beta$, are practically the same as those seen around $-500 \mathrm{~km} \mathrm{~s}^{-1}$ for the aborption of the continuum. This proves that the absorbing gas with radial velocity in the range -500 to $-100 \mathrm{~km} \mathrm{~s}^{-1}$ affects the continuum emission and the emission of the $\mathrm{H}$ I lines in the same way as will be further discussed in Sect. 4.5.

For the emission line peak at $R V=50 \pm 100 \mathrm{~km} \mathrm{~s}^{-1}$ where the normalized flux reaches a value of $\sim 10$, it should be noted that the variations seen in the difference spectra (Fig. 9) are relative changes on the 5-10\% level only. These variations must be considered with respect to the strongly varying continuum level against which the spectrum has been normalized.

\subsection{The anti-correlation between high and low radial velocity}

In Fig. 10 the temporal behaviour of the normalized flux is compared for the $\mathrm{H} \beta$ and $\mathrm{Ca}$ II jet absorptions in different velocity bins. The plotted fluxes are mean values for $R V$ intervals of $200 \mathrm{~km} \mathrm{~s}^{-1}$ widths centered at the $R V$-value indicated. The chosen velocity bins exclude spectral features like strong narrow lines which could potentially disturb the absorption depths interpretation.

The plotted curves are in principle vertical cuts through the dynamical spectra plotted in Figs. 8a and b. However, for Fig. 10 the fluxes and times from individual spectra are used and not just "daily means" as in dynamical spectra. To account for the long term velocity trend sometimes different $R V$-bins are taken for the two data series. Not included are in Fig. 10 data from some low quality spectra flagged in Table 1, namely 138 B, C, D, 184, and in the case of Ca II also 141.

$\mathrm{H} \beta$ line. Figure 10 shows that the transient jet absorption components on the high (negative) velocity side of $R V=-1900 /-2100 \mathrm{kms}^{-1}$ come and go within one or two days. Thereby the mean flux in these measuring bins seems to decrease and increase at the same speed. However this statement should be considered cautiously because the temporal resolution of our data is rather limited for constraining such fast variations. At $R V=-1500 /-1700 \mathrm{kms}^{-1}$ the jet absorption appear also rapidly and at the same time as for higher (negative) velocities, but then retreat with a significantly slower pace, typically on the time scale of about one week. The behaviour in this $R V$-range is caused by the overlap of two phenomena, the occurrence of transient high velocity components and the associated general $R V$-shift and recover of the deep absorption trough. These two variation
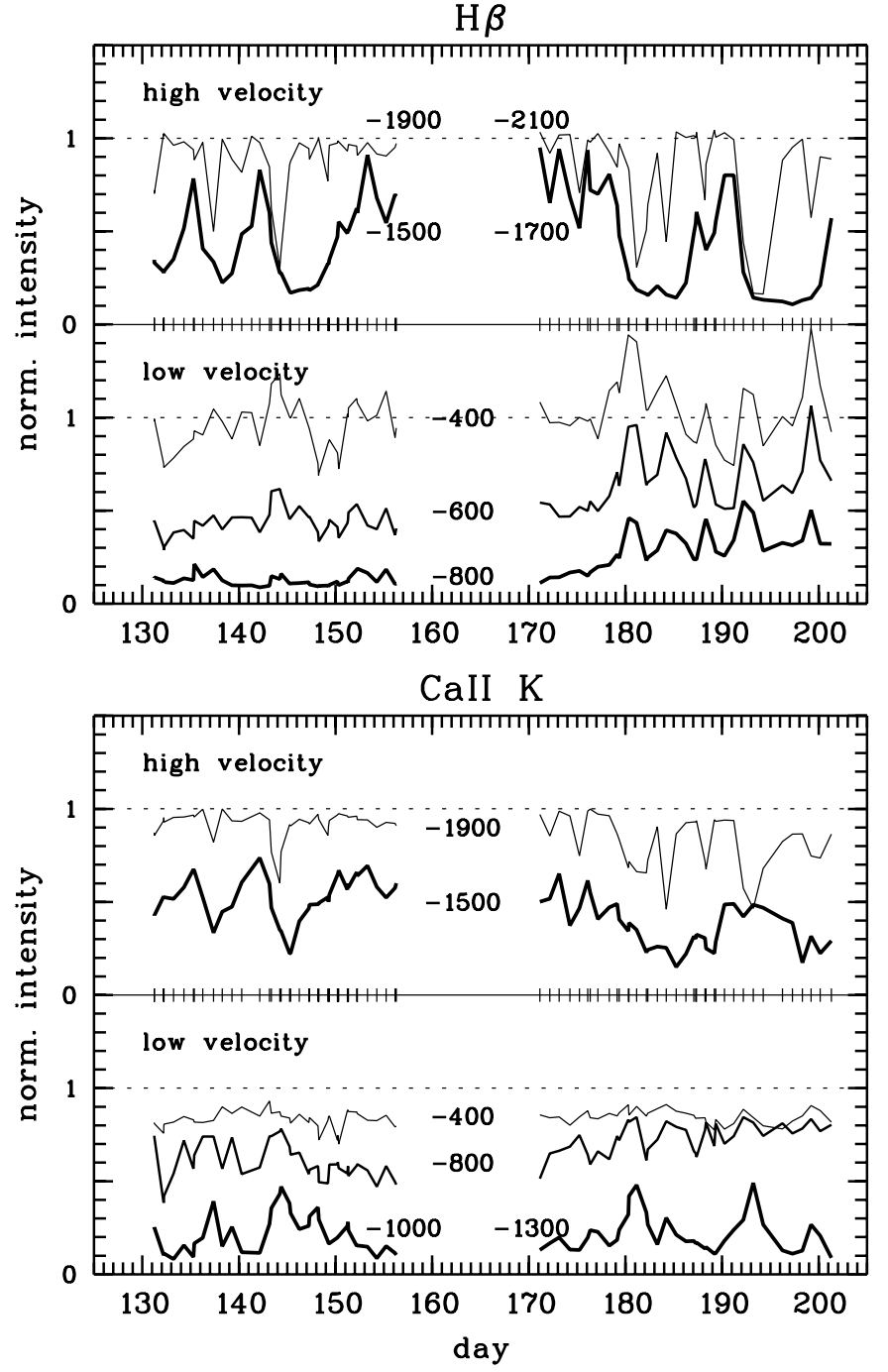

Fig. 10. Anti-correlation between the jet absorption depths at high and low (negative) velocities for $\mathrm{H} \beta$ and CaII K. Normalized fluxes at different radial velocities are given as function of time. The measured fluxes are mean values for $R V$-bins centered at the values indicated in the middle between the the first and second data series. For some line regions curves with higher (negative) $R V$-values are taken for days 171-201 (second series) due to the general $R V$-trend of the jet absorptions. The short vertical dashes along the horizontal line in the middle of the panels indicate the time of individual spectra.

types are also clearly visible in the dynamical spectrum in Fig. 8a.

The flux changes plotted in Fig. 10 for the low velocity side of the absorption trough at $R V=-800$ to $-200 \mathrm{~km} \mathrm{~s}^{-1}$ are in anti-correlation with respect to the mean fluxes in the $R V=-1900 /-2100 \mathrm{~km} \mathrm{~s}^{-1}$ intervals. This is particularly well visible for the variations between days 180 and 200, but also the excursions around days 144 and 137 are present. No systematic time-difference between the flux maxima at low velocity and flux minima at high velocity can be recognized with our temporal 
resolution. The anti-correlation is not perfect in the sense that the strength of the flux maxima at low velocities does not scales linearly with the strength of the flux minima at high velocities. But this non-perfect (anti-) match may just indicate that the description of the jet absorptions variability in terms of mean flux absorptions in given $R V$ bins is oversimplified. This is not surprising when considering a sequence of individual line profiles like in Fig. 7.

Ca II $\mathrm{K}$ line. The changes of the mean flux in the jet absorption at $R V=-1900 \mathrm{~km} \mathrm{~s}^{-1}$ of Ca II K are very similar to $\mathrm{H} \beta$. Note that for CaII in Fig. 10 the flux for $R V=-1900 \mathrm{~km} \mathrm{~s}^{-1}$ is plotted for days 171-201 (instead of $-2100 \mathrm{~km} \mathrm{~s}^{-1}$ as in $\mathrm{H} \beta$ ) due to a strong emission near $-2100 \mathrm{~km} \mathrm{~s}^{-1}$. A difference is that the transient high velocity components are less deep for Ca II when compared to $\mathrm{H} \beta$ and other $\mathrm{H}$ I lines like e.g., $\mathrm{H} \delta$ (Fig. 7).

Most interesting is the variability of Ca II at $R V=$ $-1000 /-1300 \mathrm{~km} \mathrm{~s}^{-1}$, where for $\mathrm{H}$ I the line absorption is strongly saturated. In this range the Ca II feature displays strong flux variations in clear anti-correlation with the flux changes at higher velocities. The variations at $R V=$ $-1000 \mathrm{~km} \mathrm{~s}^{-1}$ in the first data series (days 131-156) is due to a shift of the low velocity end of the absorption trough towards the blue, associated with the appearance of the high velocity components (see profiles in Figs. 6 and 7). This happens also during days 171-201.

In addition the broad jet absorptions split during days with strong high velocity components into two components leaving a distinct flux maximum in the middle around $R V=-1300 \mathrm{kms}^{-1}$. The temporary splitting disappears when the two components merge again (Fig. 7). We have plotted the corresponding fluxes for $-1300 \mathrm{~km} \mathrm{~s}^{-1}$ in Fig. 10 for days $171-201$ because this effect is particularly strong during the second data series. But also for day 144 such a line splitting is visible (see Fig. 6).

The Ca II $R V=-1000 /-1300 \mathrm{~km} \mathrm{~s}^{-1}$ flux variability pattern is also recognizable at a velocity of $-800 \mathrm{~km} \mathrm{~s}^{-1}$ and partly also for $-400 \mathrm{~km} \mathrm{~s}^{-1}$. However in this latter velocity regime the variations are much smaller than for $\mathrm{H} \beta$ and harder to measure due to the larger uncertainties in the spectrum normalization for the Ca II spectral region.

\subsection{Variability for lines of different elements}

A first impression on the similarities and differences in the jet absorptions of different elements can be gained from the dynamical spectra. Figure 11 provides a more detailed view on the flux variations for given $R V$-bins.

Striking is the high similiarity between $\mathrm{H} \delta$ and HeI $\lambda 5876$ for the flux variations in the range $R V=$ $-1700 /-1900 \mathrm{~km} \mathrm{~s}^{-1}$. Practically each wiggle seen in the $\mathrm{H} \delta$-curve is also visible in He I. A difference between the two curves is that the strong flux absorptions, e.g., around days 144,181 or 193 disappear faster for the He I line

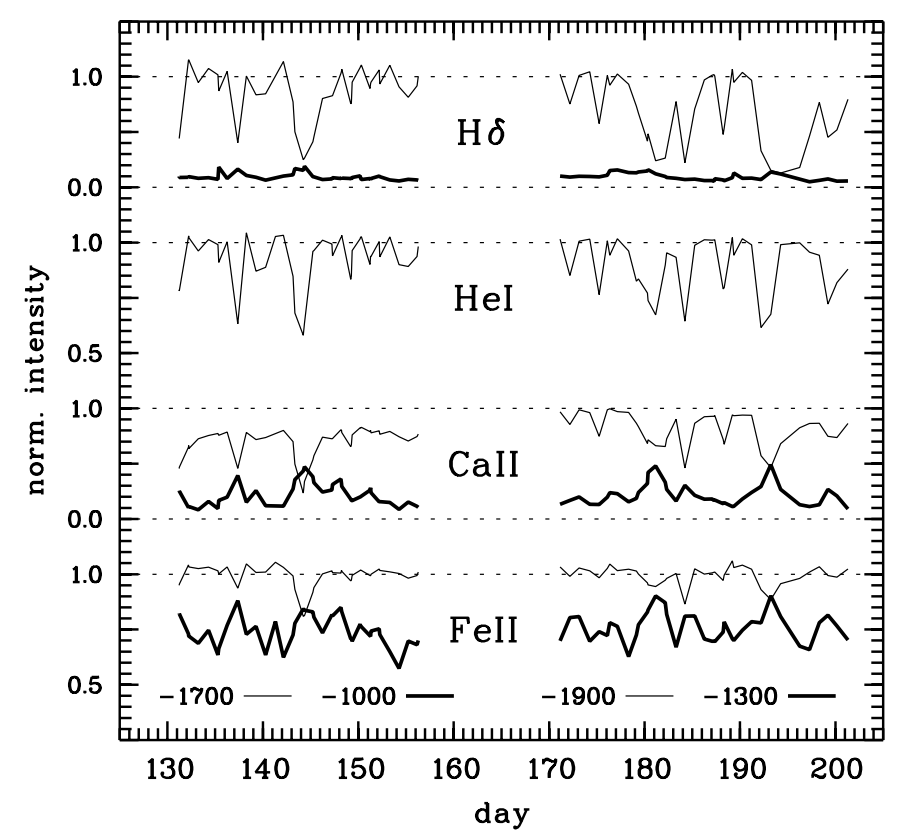

Fig. 11. Jet absorption depths as function of time for $\mathrm{H} \delta$, He I $\lambda 5876$, Ca II K and Fe II $\lambda 5169$. Normalized fluxes are given for high and low (negative) line radial velocity as indicated at the bottom of the panel. The fluxes are mean values for a $R V$-interval of $200 \mathrm{~km} \mathrm{~s}^{-1}$ widths. Curves with higher (negative) $R V$-values are taken for days 171-201 due to the general $R V$-trend of the jet absorptions. Note the different scale for HeI and Fe II compared with H I and Ca II. Due to blending with $\mathrm{Na}$ I no low $R V$-velocity curve is given for He I.

compared to the H I line. Note that the He I flux scale has been stretched in Fig. 11.

Also the CaII and Fe II flux curves show a very similar flux pattern, especially for the high velocity range $R V=-1700 /-1900 \mathrm{~km} \mathrm{~s}^{-1}$. It should be noted that the Fe II absorptions are weak and that the variations in normalized flux are only of the order $0.1-0.2$. The good match in flux variations is obtained despite the presence of narrow emission lines, or narrow absorptions in the case of CaII. Further, the Fe II-region shows the presence of a red giant continuum against which the hot component continuum (and the Fe II absorption therein) is varying due to flickering activity.

The correspondence between the CaII and Fe II flux curves for $R V=-1000 /-1300 \mathrm{~km} \mathrm{~s}^{-1}$ is also very good. Differences have to be expected for this central region of the jet absorption, because the CaII line is partly saturated there while the weaker Fe II line is not.

\subsection{Low velocity absorption and $\mathrm{HI}$ line emission}

The flux level on the low (negative) velocity side of the main absorption trough $R V \gtrsim-800 \mathrm{~km} \mathrm{~s}^{-1}$ varies in anticorrelation relative to the high velocity side. Illustrative for these spectral variations is the comparison of different Balmer lines for day 177 and day 181 in Fig. 12. Between 


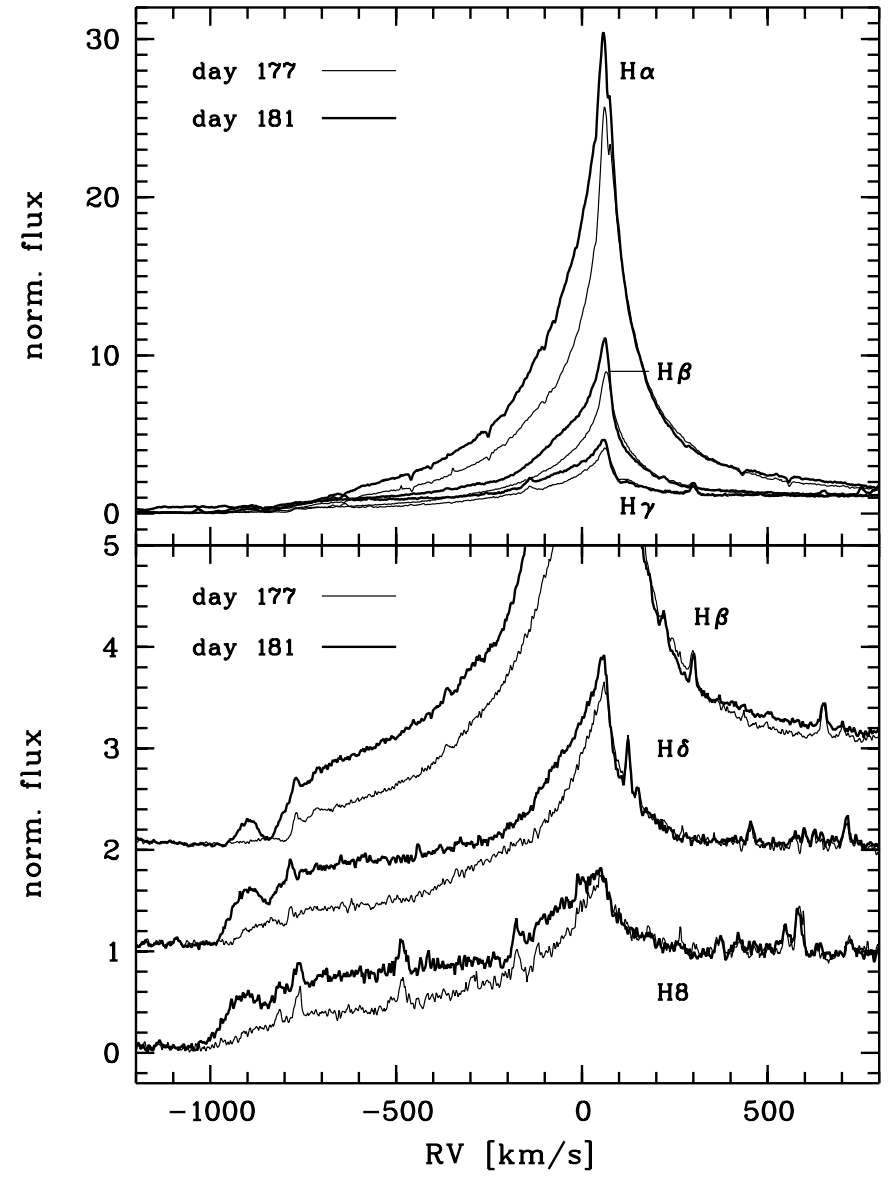

Fig. 12. Structure and variations of the $\mathrm{H}$ I line emission and the low velocity absorption in the blue line wing.

these two dates the flux level increased steadily from a low to a high value (see Figs. 10 and 11).

It is apparent that the different Balmer lines in Fig. 12 show a very similar flux increase on their blue side. This is true for the weak Balmer lines like H8, where the flux is predominantly due to the blue continuum as well as for the strong Balmer lines like $\mathrm{H} \alpha$ and $\mathrm{H} \beta$, where the emission line wings dominate. Despite the large difference in oscillator strengths (i.e. absorption cross section) the relative flux increase between day 177 and day 181 is for all Balmer lines about a factor 1.8 for $R V=-500 \mathrm{~km} \mathrm{~s}^{-1}$ where the continuum dominates and about a factor 1.4 at $-100 \mathrm{~km} \mathrm{~s}^{-1}$ where the line emission is strong (see Table 5). At the same time the flux in the red line wings remains unchanged.

This must be interpreted as a change in the covering factor of the low velocity gas outflow. The fact that continuum and the blue line wing of $\mathrm{H}$ I emission react simultaneously indicates that the corresponding emission regions coincide partly or have at least a very similar geometry. The broad $\mathrm{H} \alpha$ and $\mathrm{H} \beta$ line wings $|R V|>500 \mathrm{~km} \mathrm{~s}^{-1}$ can be attributed to Raman scattering by neutral hydrogen in the accretion disk. Raman scattering converts e.g., the $\mathrm{Ly} \beta$ and $\mathrm{Ly} \gamma$ line wing photons into $\mathrm{H} \alpha$ and $\mathrm{H} \beta$ line
Table 5. Flux in the Hi line wings compared to the continuum absorption in the blue and the integrated line flux. Fluxes $f$ (day) are line plus continuum flux normalized to the continuum. Integrated line fluxes $f$ (day) (continuum subtracted) are given in $\AA$ and are measured relative to the continuum. The last column gives the mean for the flux ratios. During day 177 no high velocity component is present and the low velocity absorption is strong while for day 181 there is a high velocity component but only weak low velocity absorption (see Figs. 12 and 7).

\begin{tabular}{|c|c|c|c|c|c|c|}
\hline day/ratio & $\mathrm{H} \alpha$ & $\mathrm{H} \beta$ & $\mathrm{H} \gamma$ & $\mathrm{H} \delta$ & $\mathrm{H} 8^{a}$ & mean \\
\hline \multicolumn{7}{|c|}{ flux at $R V=-500 \mathrm{~km} \mathrm{~s}^{-1}$ (continuum) } \\
\hline$f(177)$ & 1.48 & 0.64 & 0.46 & 0.52 & 0.46 & \\
\hline$f(181)$ & 2.26 & 1.11 & 0.91 & 0.92 & 0.82 & \\
\hline$f(181) / f(177)$ & 1.53 & 1.73 & 1.98 & 1.77 & 1.78 & 1.76 \\
\hline \multicolumn{7}{|c|}{ flux at $R V=-100 \mathrm{~km} \mathrm{~s}^{-1}$ (blue line wing) } \\
\hline$f(177)$ & 7.10 & 2.71 & 1.61 & 1.29 & 0.98 & \\
\hline$f(181)$ & 10.7 & 4.11 & 2.23 & 1.66 & 1.33 & \\
\hline$f(181) / f(177)$ & 1.51 & 1.52 & 1.39 & 1.29 & 1.36 & 1.41 \\
\hline \multicolumn{7}{|c|}{ flux at $R V=+150 \mathrm{~km} \mathrm{~s}^{-1}$ (red line wing) } \\
\hline$f(177)$ & 10.4 & 3.39 & 1.83 & 1.49 & 1.14 & \\
\hline$f(181)$ & 10.3 & 3.21 & 1.73 & 1.50 & 1.17 & \\
\hline$f(181) / f(177)$ & 0.99 & 0.95 & 0.95 & 1.01 & 1.03 & 0.99 \\
\hline \multicolumn{7}{|c|}{ integrated line flux } \\
\hline$f(177)$ & 135. & 24.1 & 7.7 & 3.6 & 1.4 & \\
\hline$f(181)$ & 174. & 32.9 & 9.7 & 5.0 & 2.1 & \\
\hline$f(181) / f(177)$ & 1.29 & 1.37 & 1.26 & 1.39 & 1.5 & 1.36 \\
\hline
\end{tabular}

$a$ : Fluxes for other Balmer lines, like $\mathrm{H} \epsilon, \mathrm{H} 9$ and H10, are not accurately measurable because of blending with narrow emission lines, CaII emission and interstellar absorption (for $\mathrm{H} \epsilon$ ) and uncertainties in the spectrum normalization (for H10, H11).

wing photons, respectively, whereby the $R V$-shift from the line center is enhanced by a factor of about 6.4 (see Nussbaumer et al. 1989). This effect is often observed in symbiotic systems, because they have strong H I Lyman line emission and dense and extended neutral hydrogen regions for Raman scattering (e.g., Lee 2000; Schmid 2001). In the case of MWC 560 the Lyman line emission from the bright disk "chromosphere" can be Raman scattered in the underlying neutral disk "photosphere".

The spectral changes described here between days 177 and 181 are characteristic for the absorption variability in the blue wing of the $\mathrm{HI}$ emission lines and the adjacent continuum although they are usually not so strong. Because the flux in the red wing of the Balmer line emission is not affected by line absorption it must change together with the strongly variable continuum flux.

For day 181 there is at $R V=-850 \mathrm{~km} \mathrm{~s}^{-1}$ an absorption component which is much weaker in H8 than e.g. in $\mathrm{H} \beta$. This is an example for an absorption with a moderate line opacity, but which covers practically the entire continuum source. 
Finally an estimate on the H I line fluxes of MWC 560 is given. Assuming an apparent brightness of roughly $m_{V}=10.5 \mathrm{mag}$ and $m_{B}=11 \mathrm{mag}$ for MWC 560 for our campaign (disregarding the flickering variations) yields a continuum flux of about 4.8 and $3.5 \times$ $10^{-13} \mathrm{erg} \mathrm{s}^{-1} \mathrm{~cm}^{-2} \AA^{-1}$ at $4500 \AA$ and $5500 \AA$, respectively. With the $E W$ for day 177 the approximate line fluxes of 1.1 for $\mathrm{H} \delta, 2.4$ for $\mathrm{H} \gamma, 6.5$ for $\mathrm{H} \beta$ and 24.3 for $\mathrm{H} \alpha$ in units of $10^{-12} \mathrm{erg} \mathrm{s}^{-1} \mathrm{~cm}^{-2}$ are obtained.

\subsection{Velocity structure of the outflow}

In MWC 560 the outflow profiles and their temporal variability differs completely from classical P Cygni profiles pointing to a very specific outflow structure. P Cygni type line profiles from stellar outflows are due to gas which is accelerated outwards from a small velocity near the source to high velocities at large distances approaching asymptotically a terminal velocity $v_{\infty}$. If variability is observed in these P Cygni profiles they can be characterized as net absorption enhancements evolving in $R V$ velocity like a discrete component in the accelerated wind (see e.g., Lamers \& Cassinelli 1999).

For the following discussion of the MWC 560 outflow absorptions we just assume that the gas is accelerated from low velocities and then reaches a high velocity at large distances. The important characteristics of the profiles observed during our campaign are:

- at low velocities $\$ 600 \mathrm{~km} \mathrm{~s}^{-1}$ there is only a shallow absorption visible indicating that there is no low velocity gas in front (or is covering only a minor fraction) of the predominant radiation source;

- the low velocity absorption seen in the blue wing of the $\mathrm{H}$ I emission lines behaves like the absorption in the continuum indicating that the acceleration region is extended and covers partly the luminous $\mathrm{H}$ I emission line region;

- the strength of the low velocity absorption is variable but shows no discrete components indicating that possible outflow inhomogeneities in the initial acceleration region are much smaller than the continuum and the $\mathrm{H}$ I emission regions lying behind it;

- only gas moving faster than $\gtrsim 700 \mathrm{~km} \mathrm{~s}^{-1}$ produces strong absorptions along the line of sight to the main continuum source. Often there are several saturated (in $\mathrm{HI}$ ) absorption components with different velocities present indicating that they are located behind each other along the line of sight;

- there appear repeatedly on a time scale of a few days transient high velocity $\approx 1500-2500 \mathrm{~km} \mathrm{~s}^{-1}$ absorption components. Their life time is about one day for minor high velocity components and several days for major components;

- the evolution of major high velocity components is characterized by a sudden appearance reaching maximum velocity within one day, followed by a continuous velocity decrease over a few days back to about $\approx 1500 \mathrm{~km} \mathrm{~s}^{-1}$;

- the appearance of a strong high velocity component is always connected with a weakening of the absorption at low velocties $<600 \mathrm{~km} \mathrm{~s}^{-1}$. This anti-correlation is simultaneous within one day (the time resolution of our data) indicating that the high velocity components originate from a region closer than about $1 \mathrm{AU}$ $(=v \cdot \Delta t)$ from the jet source (acceleration region);

- there exists always a saturated (in $\mathrm{HI}$ ) absorption component with an intermediate velocity of $\approx 900-1300 \mathrm{~km} \mathrm{~s}^{-1}$. This absorption remains even when a saturated high velocity component is present for several days. This proves that intermediate velocity gas must be located further away from the jet source than the high velocity components which are connected to the acceleration region;

- the H I emission peaks are narrow and strong line emission extends only to a few $100 \mathrm{~km} \mathrm{~s}^{-1}$ in recession velocity. Apart from the Raman scattered wings in $\mathrm{H} \alpha$ and $\mathrm{H} \beta$ there is a lack of red shifted line emission indicating that the outflow is not spherically symmetric.

Thus, compared to the $\mathrm{P}$ Cygni type outflow, MWC 560 shows a pulsed acceleration to high velocities $1500-2500 \mathrm{~km} \mathrm{~s}^{-1}$ from a low radial velocity region $<600 \mathrm{~km} \mathrm{~s}^{-1}$ located close but not in front (at most partly) of the radiation source. The high velocity gas is then decelerated to an intermediate terminal velocity of roughly $1200 \mathrm{~km} \mathrm{~s}^{-1}$. This major result follows from the observed jet absorptions in the hot continuum. Further support and additional constraints are obtained from the jet absorptions seen in front of the red giant (Sect. 5) and the narrow line region (Sect. 6).

\subsection{Time scales and mass outflow}

The spectral variations in the jet absorptions are witnessing strong changes in the mass outflow. The time scale for the appearance or disappearance of components gives thereby a measure of the life time of gas structures and estimates on the length of the probed jet along the line of sight. For example, absorbing gas with a $R V$ of $-1700 \mathrm{~km} \mathrm{~s}^{-1}$ travels within one day about $1 \mathrm{AU}$ further away from the jet source. The fact that there are often two or even more deep (saturated) absorptions with strongly different velocities present, as e.g., for day 181 in Fig. 7, proves that the gas components cover the entire continuum source and are therefore located behind each other along the line of sight.

The mass outflow per unit time and surface element can be roughly estimated from the time scale and the $E W$ changes of jet absorption structures. Strong jet components, which appear on time scales of one day, have $E W$ of up to $40 \%$ of the entire jet absorption $E W$ (see Table 2). This is also true for Fe II, although the total $E W$ as given in Table 2 changes much less than for saturated absorptions. This is because the lower velocity component is 
weakened when a high velocity component appears, due to the described anti-correlation effect. Thus the spectral variability together with the previously determined column densities (Sect. 3.1) indicate that a column density of roughly $N(\mathrm{H}) \approx 10^{23} \mathrm{~cm}^{-2}$ is replenished in $\Delta t \approx 1$ day in the jet outflow along the line of sight during the appearance of a high velocity component. Still to be determined remains the total cross section $\pi R_{\text {jet }}^{2}$ of the probed jet for an estimate on the mass outflow. However, the value of $R_{\text {jet }}$ depends strongly on the adopted system parameters and the jet geometry and will be discussed in Sect. 8.2.

\section{The $\mathrm{H} \alpha$ jet absorption in front of the red giant}

The red giant contributes about $45 \%$ to the continuum in the $\mathrm{H} \alpha$ region. In the $\mathrm{H} \alpha$ jet absorption trough the hot component is strongly obscured for $R V \approx-1500$ to $-800 \mathrm{~km} \mathrm{~s}^{-1}$ and the remaining flux is at least predominantly due to the red giant. But within this velocity range an even deeper absorption component is present which must be due to gas in front of the red giant. This absorption is very strong at the begining of our campaign and then diminishes and disappears within the 70 days covered by our data. Such an obscuration of the red giant companion by the jet could be caused by the binary motion or by a precession of the jet axis.

Figure 13 illustrates the structure and the temporal evolution of the $\mathrm{H} \alpha$ component in the red giant continuum. The spectra are normalized relative to the radial velocity interval -1000 to $-850 \mathrm{~km} \mathrm{~s}^{-1}$. This $R V$-region shows the red giant spectrum free from contamination by the hot component. It can also be said that this part of the red giant spectrum is not or at most only very slightly absorbed by the jet gas. This can be concluded from days where the transient high velocity components are deep, as for day 201 or 193, which reveal also for $R V<-1300 \mathrm{~km} \mathrm{~s}^{-1}$ the level of the red giant continuum as expected from the normalization region $R V=-1000$ to $-850 \mathrm{~km} \mathrm{~s}^{-1}$. The normalized spectrum from the comparison star S Lep (M6+ IIIa) shows that all narrow features in the absorption trough come from the red giant spectrum.

The $\mathrm{H} \alpha$ jet absorption in front of the red giant shows a very smooth evolution in contrast to the strongly variable absorption in the continuum of the hot component. The absorption is around day 133 strong and has a blue and red component centered at $R V=-1210 \mathrm{~km} \mathrm{~s}^{-1}$ and $-1105 \mathrm{~km} \mathrm{~s}^{-1}$ respectively. The red component weakens steadily from our first observation on day 133 and disappears between day 154 and 171 where no observations were made. The $E W$ of the blue component remains from day 133 to about 142 constant and then decreases steadily and vanishes around day 200. Measured line widths for the blue component are $100 \pm 10 \mathrm{~km} \mathrm{~s}^{-1}$ ( $F W H M$ ) up to day 156. From day 171 onwards the widths is $55 \mathrm{~km} \mathrm{~s}^{-1}$ and the center of the profile has slightly shifted to $-1186 \mathrm{~km} \mathrm{~s}^{-1}$. The width of the red component is about $50 \mathrm{~km} \mathrm{~s}^{-1}$.

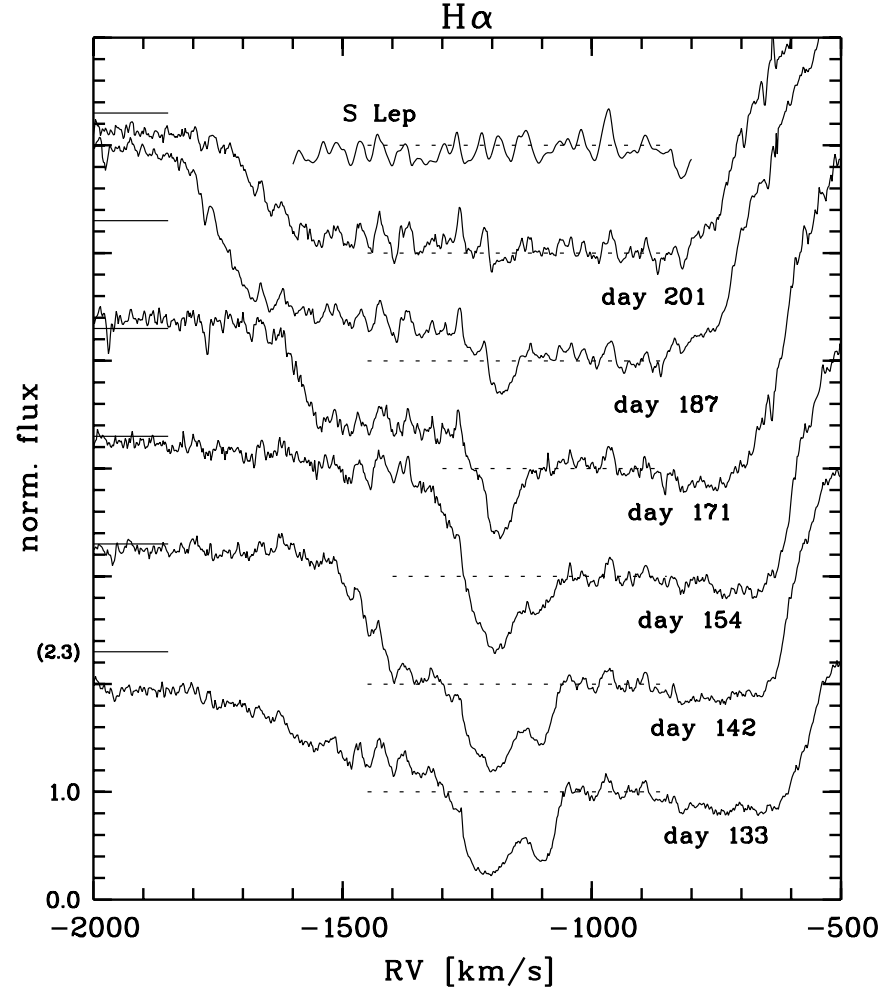

Fig. 13. Time evolution of the $\mathrm{H} \alpha$ jet absorption component in front of the red giant. The spectra are normalized to the practically unobscured red giant continuum between -1000 to $-850 \mathrm{~km} \mathrm{~s}^{-1}$. For clarity spectra are shifted vertically by integer units. The dotted line gives the adopted continuum of the red giant against which the $\mathrm{H} \alpha$ absorption in the region -1250 to $-1050 \mathrm{~km} \mathrm{~s}^{-1}$ is measured. For comparison purposes a section of the spectrum from the comparison star S Lep (M6+ IIIa) is included at the top. The short solid lines at left mark for the individual spectra a flux of 2.3 in order to illustrate the flux variations of the hot continuum.

Table 6 lists for some dates the equivalent widths and the residual central intensity relative to the red giant continuum for the blue and red component. Thereby we have split the total line equivalent widths into a blue and red value according to the strengths of the two line components. The total line equivalent widths is just the sum of these two values. For day 171 and later, when the red component is absent, only values for the blue component are given.

A search for similar jet absorptions in the red giant spectrum for other strong transitions was unsuccessful. In particular no signatures were found in the region of the Na I resonance lines and the Ca II triplet.

It is suspected that this obscuration event could be a periodic phenomenon, because the evolution of the line strengths is similar to the egress phase of an eclipse curve. Further a similar event with a blue shifted absorption in the spectrum of the red giant was reported for April 1990 by Meier et al. (1996). 
Table 6. Equivalent widths $E W$ and residual central line intensity $I_{\mathrm{c}}$ for the blue and red components of the $\mathrm{H} \alpha$ jet absorption in front of the red giant. The total line equivalent widths is $E W_{\text {blue }}+E W_{\text {red }}$. The parameters refer to the flux normalization with respect to the red giant spectrum as described in the text and Fig. 13. $E W$ are given in $\AA$.

\begin{tabular}{ccccc}
\hline day & \multicolumn{2}{c}{ blue } & \multicolumn{2}{c}{ red } \\
& $E W$ & $I_{\mathrm{c}}$ & $E W$ & $I_{\mathrm{c}}$ \\
\hline 131 & 1.76 & 0.26 & 0.98 & 0.34 \\
133 & 1.79 & 0.24 & 0.85 & 0.36 \\
137 & 1.70 & 0.25 & 0.74 & 0.42 \\
142 & 1.74 & 0.20 & 0.70 & 0.43 \\
148 & 1.57 & 0.28 & 0.50 & 0.58 \\
154 & 1.24 & 0.32 & 0.39 & 0.65 \\
171 & 0.76 & 0.38 & $<0.10$ & - \\
179 & 0.54 & 0.46 & - & - \\
187 & 0.31 & 0.71 & - & - \\
194 & 0.22 & 0.81 & - & - \\
201 & $<0.10$ & - & - & - \\
err. & \pm 0.07 & \pm 0.02 & \pm 0.07 & \pm 0.02 \\
\hline
\end{tabular}

Column densities and terminal velocity. The fact that the residual flux of the $\mathrm{H} \alpha$ jet absorption in the spectrum of the red giant is only about 0.25 for days 131 to about 150 is clear evidence that the outflow obscures a significant fraction of the red giant. Thus the extend of the absorbing outflow must be at least similar to the diameter of the red giant, which is of the order of $1 \mathrm{AU}$. From the equivalent widths given in Table 6 the required mean column density is $N(\mathrm{H} \mathrm{I}, n=2) \gtrsim 1.0 \times 10^{13} \mathrm{~cm}^{-2}$ (formula in Sect. 3.1 with $f_{j k}=0.64$ for $\mathrm{H} \alpha$ ). This is a lower limit because incomplete coverage of the red giant by an inhomogeneous gas distribution can produce the same $E W$ for a much higher mean column density.

Still, there remains the principle problem that it is difficult to estimate the total hydrogen column density $N(\mathrm{H})$ because of the unknown population of the level $n=2$.

The $\mathrm{H} \alpha$ jet absorption in the red giant spectrum can be considered as cross section through the jet at large distance from the source. The measured velocity of the two components at the systemic $R V$ of -1250 and $-1140 \mathrm{~km} \mathrm{~s}^{-1}$ can thereby be considered as terminal velocity $v_{\infty}$ of the jet outflow. The two velocity components and the fact that one component disappears before the other component indicates that the jet has also at larger distance from the source $(\gtrsim 10 \mathrm{AU})$ an internal velocity structure.

\section{The narrow line emission region}

Many hundreds of narrow emission lines are visible in the optical spectrum of MWC 560. From these lines we can estimate physical parameters of the emitting gas and investigate whether and how the jet absorptions occult the corresponding emission line region.
Most strong narrow lines are allowed transitions from singly ionized heavy elements, mainly from Fe II, Ti II and others. In addition there are lines from allowed transitions of neutral metals, most notably Fe I and Mg I. The emissions from the resonance doublets of Ca II and $\mathrm{NaI}$ are also present but strongly affected by interstellar absorption components. Forbidden lines are seen from [O I] and [FeII].

Identifications for many of these lines are given in Kolev \& Tomov (1993) and Chentsov et al. (1997).

Due to the large number of lines we discuss here only the properties of some selected lines in order to gain insight in physical parameters and the location of the emitting gas.

\subsection{Emission line diagnostics}

Allowed line region. Here we consider the region emitting the allowed lines of ionized heavy elements such as Fe II, TiII, CrII and others. This region is hereafter simply called the Fe II region. Further we assume that the allowed lines of neutral atoms are emitted at least partly in the same volume but may also extend further into the neutral regime. For our discussion we consider mainly the properties of the Fe II line spectrum, because the formation of Fe II lines under non-LTE conditions has been studied in much detail (see Viotti et al. 1988).

The formation of Fe II emission lines depends strongly on often rather badly known parameters like radiation field, line optical depths, Fe II abundance, electron density and gas temperature. It is beyond the scope of this paper to make a detailed analysis of these parameters based on the narrow line spectrum. However, based on numerical calculations on the Fe II emission line spectrum given in the literature (e.g., Netzer \& Wills 1983; Joly 1981; Verner et al. 1999) a few rough estimates can be made from the basic properties of the Fe II emission line spectrum in MWC 560.

First, it can be concluded that Fe II is emitted in MWC 560 in a partially ionized region $N(\mathrm{H}$ II $) / N(\mathrm{HI}) \lesssim$ 0.1 . This follows from the presence of the strong line spectrum of the Ti II ion, which has practically the same ionization potential as H I. Strong ionization of hydrogen would also cause ionization (and depletion) of $\mathrm{Ti}$ II in conflict with the narrow line spectrum.

A partially ionized Fe II-region can also explain the fast Fe II flux variations to be described in the following sections. The Fe II lines show absolute flux variations of up to a factor of 2 within approximately one day simultaneously with the continuum flux. Such variations cannot be due to radiative excitation because the absorption profiles are narrow and the line depths for the Fe II UV transitions are high (see below). However, if the continuum radiation changes the electron density $N_{\mathrm{e}}$, e.g., by a factor of 2, as response to the photoionization of $\mathrm{HI}$, then an immediate change of the Fe II emission line flux $F \propto N_{\mathrm{e}} N($ Fe II $) \exp \left(-\chi / k T_{\mathrm{e}}\right)$ in step with the variation of 
the ionizing flux is possible ( $\chi$ : required excitation potential for the emitted line; $k$ : Boltzmann constant).

A rough upper limit on the electron temperature of $T_{\mathrm{e}} \lesssim 12000 \mathrm{~K}$ follows from the requirement that hydrogen is predominantly neutral in the Fe II region. For higher temperatures hydrogen would be substantially ionized by electron collisions (e.g., Arnaud \& Rothenflug 1985). However, under many different astrophysical conditions the temperature in partially neutral $N(\mathrm{H}$ II $) / N(\mathrm{HI}) \lesssim 0.1$ regions emitting Fe II is below $T_{\mathrm{e}}<10000 \mathrm{~K}$.

A lower limit on the electron temperature $T_{\mathrm{e}}$ in the Fe II region can be made from the presence of the many strong optical Fe II emission lines. The formation of these lines requires that the lowest odd level of Fe II at about $5 \mathrm{eV}$ are readily excited. For this a minimum electron temperature of about $T_{\mathrm{e}} \gtrsim 7000 \mathrm{~K}$ is needed for collisional excitation (see e.g., Netzer 1988). Radiative excitation of the odd levels at $5 \mathrm{eV}$ by continuum radiation is also possible but most likely not efficient in MWC 560, because the absorbing (i.e. the emitting) gas has only narrow lines and can therefore absorb only a limited amount of UV radiation. Further we can assume that the covering factor of the continuum radiation by the (stationary) narrow line Fe II gas is much lower than unity in view of the presence of a strong polar outflow region.

The mean electron density $N_{\mathrm{e}}$ of the Fe II emitting gas is larger than $N_{\mathrm{e}} \gtrsim 10^{7} \mathrm{~cm}^{-3}$ as can be inferred from the weakness of the forbidden [Fe II] compared to allowed Fe II lines. A similar constraint on $N_{\mathrm{e}}$ is obtained from the short time scale of $\sim 1$ day for the Fe II line variations. We assume as suggested above that this variability is caused by changes in the hydrogen ionization degree in the Fe II region due to the variable ionizing flux from the accreting object. The corresponding recombination time scale $t_{\text {rec }}$ for H II is given by $t_{\text {rec }} \approx 1 / \alpha N_{\mathrm{e}}$, where $\alpha$ is the radiative $\mathrm{H}$ II recombination cross section $\left(\alpha_{B}(8000 \mathrm{~K})=\right.$ $\left.3 \times 10^{-13} \mathrm{~cm}^{3} \mathrm{~s}^{-1}\right)$. Thus, the observational requirement that $t_{\text {rec }} \lesssim 1$ day yields $N_{\mathrm{e}} \gtrsim 4 \times 10^{7} \mathrm{~cm}^{-3}$.

For the total hydrogen density $N(\mathrm{H})$ it must be considered that a low ionization fraction $N(\mathrm{HII}) \approx N_{\mathrm{e}} \lesssim$ $0.1 N(\mathrm{HI})$ is adopted for the Fe II region. This yields from the estimated $N_{\mathrm{e}}$ above a lower limit for the hydrogen density of at least $N(\mathrm{H}) \gtrsim 4 \times 10^{8} \mathrm{~cm}^{-3}$. However, it must be supposed that the density could be in reality orders of magnitudes higher.

It is well known that line optical depths can significantly change the flux ratios between optical and UV transitions of Fe II (e.g., Netzer 1988). For MWC 560, Michalitsianos et al. (1991) report narrow absorptions but no strong narrow emission lines from UV (IUE) satellite observations. This suggests that the optical depths in the strong UV transitions of Fe II are so high that UV line photons are converted into optical Fe II line photons after multiple scatterings.

In summary, the parameters of the partially ionized Fe II region are characterized by a temperature in the range $T_{\mathrm{e}} \approx 7000-10000 \mathrm{~K}$, particle densities larger (most likely much larger) than $N_{\mathrm{e}} \gtrsim 4 \times 10^{7} \mathrm{~cm}^{-3}$ and $N(\mathrm{H}) \gtrsim 4 \times 10^{8} \mathrm{~cm}^{-3}$ and substantial optical depths in the UV line transitions of Fe II. Further it is assumed that the Fe II line variations are caused by strong changes in the ionization degree $N(\mathrm{HII}) / N(\mathrm{HI}) \cong N_{\mathrm{e}} / N(\mathrm{HI})$ in the Fe II region due to the variable ionizing flux from the accreting object.

Forbidden line region. The presence of forbidden lines indicates already that there exists a lower density region with $N_{\mathrm{e}} \lesssim 10^{7} \mathrm{~cm}^{-3}$. The density $N_{\mathrm{e}}$ can be estimated from the [O I] line ratio $\lambda 5577 / \lambda 6300$ (Hamann 1994). For MWC 560 the line ratio $R(\lambda 5577 / \lambda 6300)=0.15 \pm 0.05$ is derived based on the measured equivalent widths and assuming a flat continuum $f_{\lambda}$ (the $[\mathrm{O}$ I $]$ sky lines were subtracted). This [O I]-ratio indicates a density of $N_{\mathrm{e}} \approx 10^{6}$ to $10^{7} \mathrm{~cm}^{-3}$ for the [O I]-region for temperatures in the range $T_{\mathrm{e}}=8000$ to $15000 \mathrm{~K}$.

Further it can be inferred from the absence of [N II], [S II] and [O II] lines that the ionization fraction in the forbidden line region is low. The absence of the [N II] lines indicates that the gas temperature is $T_{\mathrm{e}}<15000 \mathrm{~K}$. For higher temperatures strong $[\mathrm{N}$ II $]$ lines $(R([\mathrm{~N} \mathrm{II}] /[\mathrm{O} \mathrm{I}]) \gtrsim 1)$ are expected according to emission line calculations for a gas in collisional ("coronal") ionization equilibrium (see e.g., Hamann 1994).

\subsection{Spectral structure of the narrow lines}

The narrow line spectrum in MWC 560 is very rich and many lines are blended. Therefore we have carefully selected some representative, unblended lines in order to study the profile structures of forbidden and allowed transitions from neutral and singly ionized atoms.

Almost all narrow lines have a Gaussian structure. Some of the strongest Fe II lines are exceptions, as they display an extended blue line wing (Fig. 14). Exceptions are also the CaII triplet lines which are double-peaked.

Table 7 gives line centers and radial velocity widths $(F W H M)$ for representative lines. These parameters are obtained by fitting Gaussian profiles to the lines observed on day 153. No significant temporal changes $\left(>2 \mathrm{~km} \mathrm{~s}^{-1}\right)$ are detected in line widths and centers during our campaign. However, line strengths are variable (see next section). We measure a radial velocity of $+36 \pm 1 \mathrm{~km} \mathrm{~s}^{-1}$ for the line centers of all selected lines. The line widths differ between different line types. For forbidden [O I] and [Fe II] lines as well as for allowed Fe I lines the widths are $16 \pm 1 \mathrm{~km} \mathrm{~s}^{-1}$. The $\mathrm{Mg}$ I $\lambda 5172.68$ line is significantly broader with a $F W H M$ of $25 \pm 1 \mathrm{~km} \mathrm{~s}^{-1}$. Although the other strong $\mathrm{Mg}$ I features of the multiplets 2 and 3 are blended or located in the wing of another strong line, they confirm this result.

The allowed Fe II lines show a range in line widths and blue wing strengths. The very strong FeII lines from multiplet 42 are broad $\left(F W H M \approx 40 \mathrm{kms}^{-1}\right)$ and have pronounced blue wings extending more than $100 \mathrm{~km} \mathrm{~s}^{-1}$ towards the jet absorption component. Other 


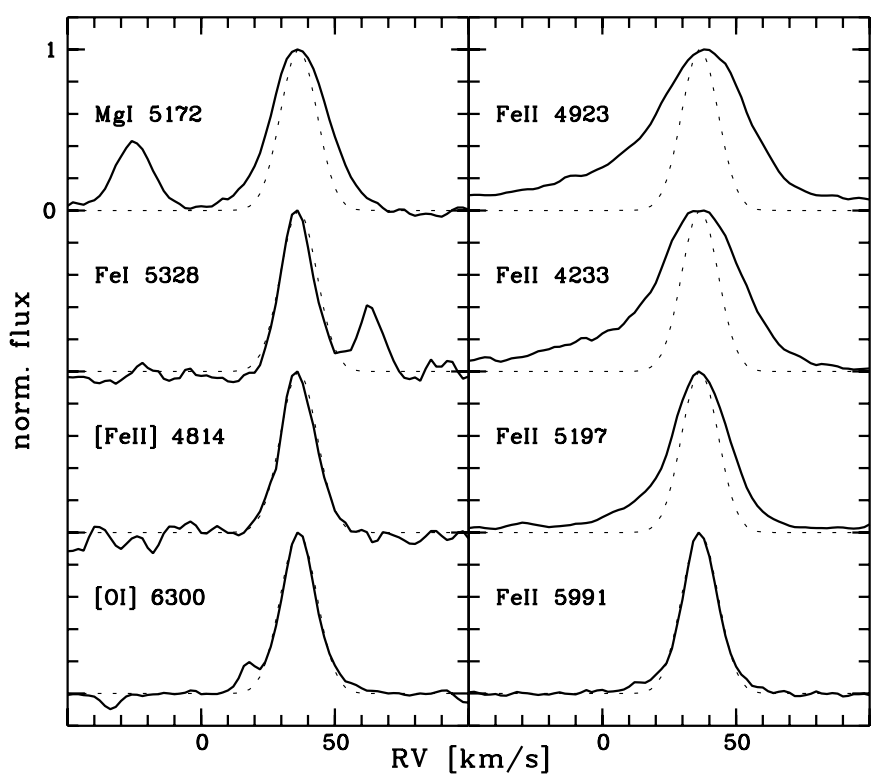

Fig. 14. Spectral structure of selected narrow emission lines in MWC 560 (day 153). The dotted line is a Gaussian profile centered at $R V=+36.5 \mathrm{~km} \mathrm{~s}^{-1}$ and a width of $16 \mathrm{~km} \mathrm{~s}^{-1}$ $(F W H M)$. The weak emission component at $+18 \mathrm{~km} \mathrm{~s}^{-1}$ in $[\mathrm{OI}]$ is due to the night sky emission of [O I]. The lines are nomalized so that the continuum corresponds to 0 and the peak flux to 1 . In addition, the profiles are shifted by integer units for clarity.

strong FeII lines with traces of a jet absorption component, like Fe II $\lambda 4233.17$, show a similar profile with a somewhat weaker blue wing. Further there are strong Fe II lines with a width in the range $20-30 \mathrm{~km} \mathrm{~s}^{-1}$ and a very weak blue extension. However, the majority of lines from singly ionized atoms have Gaussian profiles with a width like the neutral $\mathrm{Fe}$ I lines and the forbidden lines.

The Ca II triplet lines are strong, broad ( FWHM $40-60 \mathrm{~km} \mathrm{~s}^{-1}$ ), and double peaked. Their equivalent widths is about $1-2 \AA$, relative to the strong red giant continuum in this spectral region. The two CaII transitions at $\lambda 8542.09$ and $\lambda 8662.14$ have very similar profiles with a weaker flux maximum at $R V \approx+20 \mathrm{~km} \mathrm{~s}^{-1}$, a stronger maximum at $+51 \mathrm{~km} \mathrm{~s}^{-1}$, and a central minimum at $+33 \mathrm{~km} \mathrm{~s}^{-1}$. The profile of the third Ca II line at $\lambda 8498.02$ differs significantly. The two flux maxima have practically equal strengths and are located at +28 and $+44 \mathrm{~km} \mathrm{~s}^{-1}$, with the central mimimum at $+37 \mathrm{~km} \mathrm{~s}^{-1}$ close to the systemic velocity. The fact that the profiles of the two transitions $\lambda 8498$ and $\lambda 8542$ from the same upper level $\left(4 \mathrm{p}^{2} \mathrm{P}_{3 / 2}^{\mathrm{o}}\right)$ differ strongly indicates that radiative transfer affects the line structure of these transitions significantly. Certainly, the Ca II lines have a significant diagnostic potential. However, in order to get reliable information detailed radiative transfer calculations are required for the narrow line region what is beyond the scope of this paper.
Table 7. Parameters for different types of narrow emission lines. The selected lines represent different line groups: forbidden lines of neutral ([O I $]$ ) and singly ionized ([Fe II]) atoms, and allowed lines from neutral (MgI, FeI) and ionized (FeII) atoms. Line center and widths are given for day 153 .

\begin{tabular}{|c|c|c|c|c|c|}
\hline $\begin{array}{l}\lambda_{\text {lab }} \quad \text { ident. } \\
{[\AA]}\end{array}$ & $\begin{array}{c}\text { center } \\
{\left[\mathrm{km} \mathrm{s}^{-1}\right]}\end{array}$ & $\begin{array}{l}\text { width } \\
{\left[\mathrm{km} \mathrm{s}^{-1}\right]}\end{array}$ & $\begin{array}{c}\left\langle E W_{\mathrm{em}}\right\rangle \\
{[\AA]}\end{array}$ & $\begin{array}{c}\sigma_{E W} \\
{[\%]}\end{array}$ & $\begin{array}{c}\sigma_{f_{\ell} / f_{\mathrm{rg}}} \\
{[\%]}\end{array}$ \\
\hline $6300.30[\mathrm{O} \mathrm{I}]^{a}$ & +36.5 & 15.6 & 0.73 & 12.1 & 7.2 \\
\hline 4814.55 [Fe II] $20 \mathrm{~F}$ & +36.2 & 16.0 & 0.19 & $18.9^{b}$ & $12.0^{b}$ \\
\hline 5158.81 [Fe II] $19 \mathrm{~F}$ & +35.0 & 15.0 & 0.25 & $18.6^{b}$ & $11.1^{b}$ \\
\hline 5172.68 Mg I 3 & +36.7 & 25.0 & 0.55 & 11.3 & 9.6 \\
\hline 5269.54 Fe I 15 & +35.9 & 16.2 & 0.17 & $11.1^{b}$ & $14.2^{b}$ \\
\hline 5328.04 Fe I 15 & +35.9 & 15.1 & 0.14 & $12.4^{b}$ & $15.6^{b}$ \\
\hline 4923.92 Fe II 42 & +36.6 & 37.9 & 2.64 & 7.8 & 10.8 \\
\hline 5284.10 Fe II 41 & +36.9 & 18.1 & 0.82 & 5.7 & 12.9 \\
\hline
\end{tabular}

$a$ : The well separated [O I] night sky line was clipped before measuring line parameters; $b$ : weak line, where photon noise contributes to the observed scatter in line strengths.

\subsection{Variability}

Line strengths $f_{\ell}$ are measured relative to the continuum $f_{\ell} / f_{\mathrm{c}}$ (where $f_{\mathrm{c}}=1$ ) and called hereafter emission line equivalent widths $\left(E W_{\mathrm{em}}\right)$. For absolute line flux variations the flux of the variable continuum must be considered. This can be done by scaling the measured $E W_{\mathrm{em}}$ to the unobscured red giant flux in the $\mathrm{H} \alpha$ absorption trough according to $f_{\ell} / f_{\mathrm{rg}}$.

The $E W_{\mathrm{em}}$ of allowed transitions of singly ionized atoms like FeII, but also TiII, CrII and others, show only small relative variations $\sigma_{E W}=6-9 \%$ (Table 7 or Fig. 15). The expected $\sigma_{E W}$ scatter from photon shot noise is of the order of $1 \%$ for strong narrow lines with $E W_{\mathrm{em}} \approx 1 \AA$ and $5 \%$ or larger for weak lines $E W_{\mathrm{em}}<0.2 \AA$.

However, compared to the relative continuum variations of $23 \%$ (Sect. 2.4) due to the flickering hot component, the relative line flux variations $\sigma_{E W}$ of less than $10 \%$ are small. Thus to a first approximation the line fluxes for the singly ionized metal lines change in step with the strong continuum flux variations.

For the allowed lines of FeI and Mg I the scatter is enhanced with a relative value of $\sigma_{E W}=10-12 \%$.

Much larger $\sigma_{E W} \approx 19 \%$ are the variations for the forbidden [Fe II] lines $\lambda 4815$ and $\lambda 5159$. Also enhanced is the scatter for the [O I] $\lambda 6300$ line in the red (Fig. 15), especially when considering the significant contribution of about $\sim 30 \%$ from the non-variable red giant to the total continuum at this wavelength.

Measuring the line strengths $f_{\ell} / f_{\mathrm{rg}}$, relative to the continuum of the red giant as measured in the broad $\mathrm{H} \alpha$ absorption trough (Sect. 2.4) yields a different picture. The relative variations $\sigma_{f_{\ell} / f_{\mathrm{rg}}}$ are much lower for the 


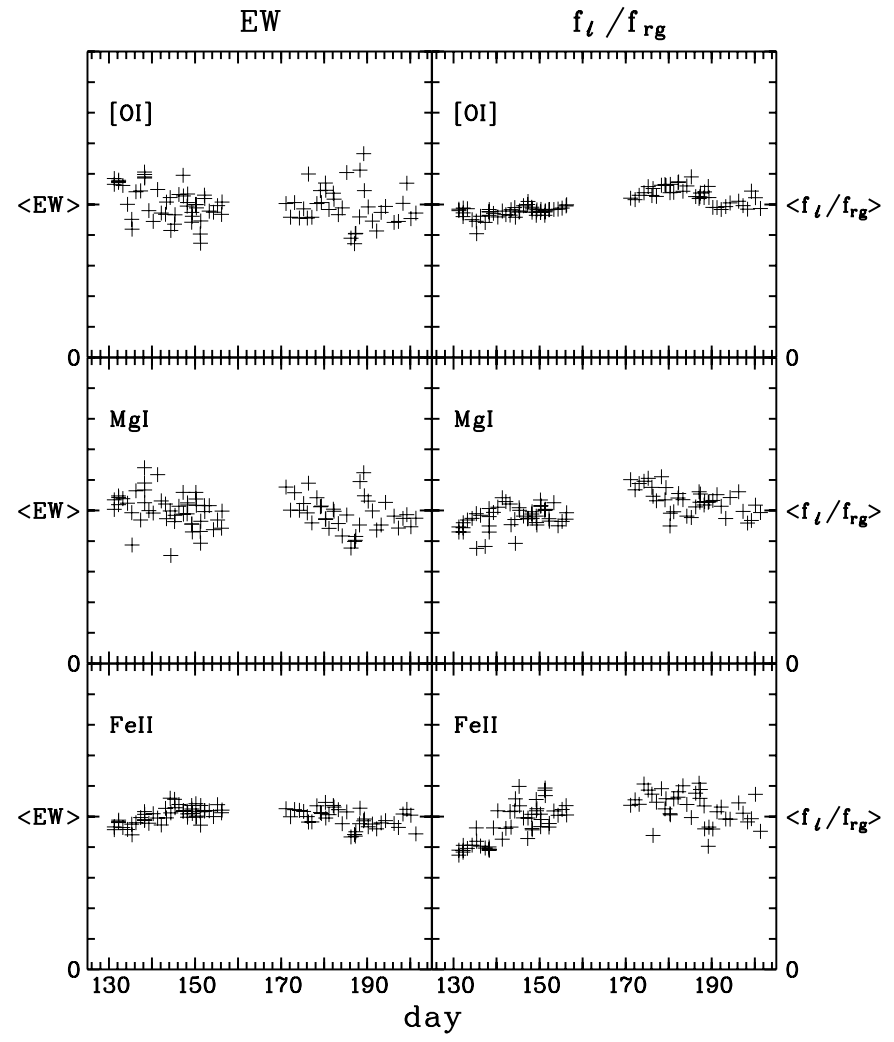

Fig. 15. Variations of the narrow emission lines [O I $] \lambda 6300$, $\mathrm{Mg}$ I $\lambda 5173$ and Fe II $\lambda 5284$. Equivalent widths $E W$ (left) are line strength relative to the total (mainly hot) continuum. $f_{\ell} / f_{\mathrm{rg}}$ (right) is the line flux relative to the continuum flux of the red giant measured in the $\mathrm{H} \alpha$ absorption trough at $6542.5 \AA$. In all panels the ordinate axis is from 0 to twice the mean value $\langle E W\rangle$ or $\left\langle f_{\ell} / f_{\mathrm{rg}}\right\rangle$. Standard deviations are given in Table 7 .

[O I] $\lambda 6300$ compared to Mg I and Fe II (Table 7 or Fig. 15). The case for [Fe II] and $\mathrm{Fe}$ I is less clear, probably because they are weak and more affected by measuring uncertainties. But for Fe I the scatter is larger for fluxes measured relative to the constant cool giant continuum compared to fluxes measured relative to the variable continuum (mainly) due to the hot component. The opposite is true for the [Fe II] lines. Fe I behaves more like Fe II and [Fe II] more like $[\mathrm{O} \mathrm{I}]$

Thus the following trend can be recognized. The absolute flux of the $[\mathrm{O} \mathrm{I}]$ lines is practically constant like the continuum of the red giant. [Fe II] and Mg I vary only slightly and may be composed of a main constant component with a contribution of a variable component, while the Fe I, the Fe II and other allowed lines of singly ionized atoms vary predominantly like the hot component. This can be interpreted as a clear stratification between allowed transitions originating mainly in an emission region varying in step with the hot component and the forbidden lines with rather constant flux. This variability pattern indicates also that the Fe II region is relatively compact and close to the continuum source, while the $[\mathrm{O}$ I]-region is much more extended. Thereby the [O I] emission may originate from a region not directly associated with the hot continuum source.

\subsection{Narrow lines in the jet absorptions}

In this section we investigate whether and how the narrow line emission region is occulted by the blue shifted absorptions from the jet gas. That some occultation is present can already be suspected from the spectral distribution on narrow lines, which shows pronounced gaps on the blue side of the strong Balmer emission lines $\mathrm{H} \beta$ to $\mathrm{H} 8$ (see Fig. 1). The partial obscuration of narrow lines by the variable jet absorptions was already noticed by Tomov \& Kolev (1997) and can be clearly seen on published spectra in Kolev \& Tomov (1993) and Tomov et al. (1997). However these studies give no assessment of this effect. In the following we investigate the occultation of the narrow line region with three different methods in order to make a quantitative statement.

Line fluxes and branching ratios. The occultation can be estimated from a comparison of observed emission line ratios for transitions with a common upper level and branching ratios from the literature. The main problems of this method are the availability of accurate atomic data and the possible presence of radiative transfer effects. We found a relative large scatter (of the order 0.5 dex) between observed line ratios and available branching ratios for lines where no occultation by the jet lines of MWC 560 is expected. The branching ratios for our study where calculated from the semi-empirical oscillator strengths taken mainly from the tables of Kurusz \& Bell (1995). For Cr II and Ti II lines we relied on the the evaluation of $\log g f$ values of Sigut \& Landstreet (1990) and the compilation of Savanov et al. (1990) respectively.

Fortunately the measured line ratios and branching ratios agree significantly better (scatter $\sim 0.25$ dex) between lines from multiplet $\mathrm{Cr}$ II $30\left(\mathrm{a}^{4} \mathrm{~F}-\mathrm{z}^{4} \mathrm{~F}^{\circ}\right)$ near $\mathrm{H} \beta$ and multiplet CrII $43\left(\mathrm{~b}^{4} \mathrm{~F}-\mathrm{z}^{4} \mathrm{~F}^{\circ}\right)$ around $5300 \AA$, having the identical upper level and the same $J$ and $L$ quantum numbers for the lower level, e.g. for ratios like $F(\lambda 4824.13) / F(\lambda 5237.34)$ which refers to $F\left(\mathrm{a}^{4} \mathrm{~F}_{9 / 2}-\mathrm{Z}^{4} \mathrm{~F}_{9 / 2}^{\mathrm{o}}\right) / F\left(\mathrm{~b}^{4} \mathrm{~F}_{9 / 2}-\mathrm{z}^{4} \mathrm{~F}_{9 / 2}^{\mathrm{o}}\right)$. The same is found for corresponding line ratios between multiplet Ti II $41\left(\mathrm{a}^{4} \mathrm{P}-\mathrm{z}^{4} \mathrm{D}^{\circ}\right)$ lines near $\mathrm{H} \gamma$ and multiplet Ti II 61 $\left(\mathrm{b}^{4} \mathrm{P}-\mathrm{z}^{4} \mathrm{D}^{\circ}\right)$ lines around $4400 \AA$. We suspect that these ratios are better suited for this analysis because systematic errors in the atomic data and radiative transfer effects may be comparable for transitions having an identical upper level and the same quantum numbers for the lower levels. Such special line ratios are rather rare and it is a fortunate coincidence that useful lines occur at the position of the expected $\mathrm{H} \beta$ and $\mathrm{H} \gamma$ jet absoptions.

From this analysis we find that the CrII 30 lines $\lambda 4848.24\left(R V_{\text {obs }}(\mathrm{H} \beta)=-767 \mathrm{~km} \mathrm{~s}^{-1}\right)$, $\lambda 4856.19\left(-280 \mathrm{~km} \mathrm{~s}^{-1}\right), \lambda 4864.32\left(+220 \mathrm{~km} \mathrm{~s}^{-1}\right)$ near $\mathrm{H} \beta$ 
$\mathrm{H} \beta$-region
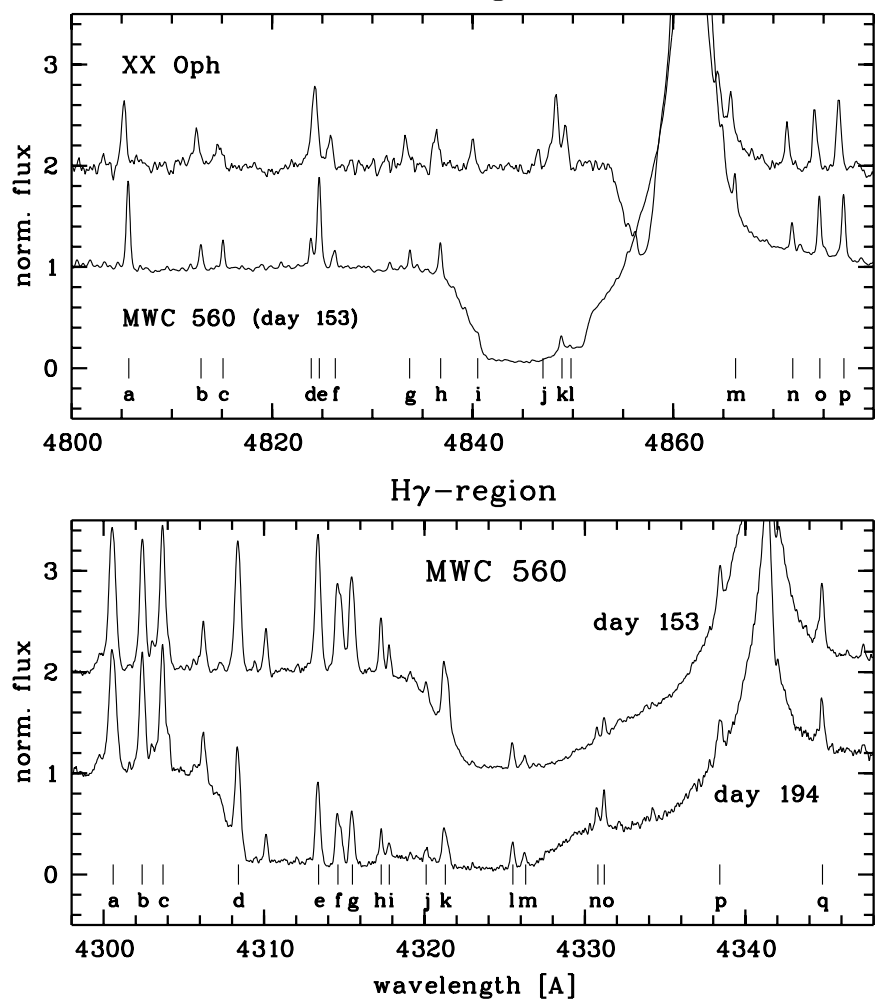

Fig. 16. Attenuation of narrow lines in the absorption troughs. The top panel compares the strengths of the narrow lines near $\mathrm{H} \beta$ in XX Oph and MWC 560 (day 153). The bottom panel shows a comparison of the $\mathrm{H} \gamma$-region for MWC 560 between day 153 and day 193, without and with a very strong high velocity absorption component.

and the Ti II 41 line $\lambda 4330.71\left(R V_{\text {obs }}(\mathrm{H} \gamma)=-639 \mathrm{~km} \mathrm{~s}^{-1}\right)$ near $\mathrm{H} \gamma$ are about a factor $5-10$ weaker than expected from the branching ratios. Other lines from the multiplets CrII 30 and TiII 41 are not weakened during days like day 153 when the jet absorption trough in the continuum is narrow and extends only from about $R V=-1300 \mathrm{~km} \mathrm{~s}^{-1}$ to $-600 \mathrm{~km} \mathrm{~s}^{-1}$. Obscurations of lines by high velocity absorption components occur but this effect is more easily studied in terms of line flux variations (see below).

Thus, it can be concluded that the jet gas obscures also the narrow line region, at least for the $R V$-range -800 to $-600 \mathrm{~km} \mathrm{~s}^{-1}$. No suitable lines can be used to test the $R V$-region from -1300 to $-800 \mathrm{~km} \mathrm{~s}^{-1}$ with this method. However there are many lines with $R V<-1300 \mathrm{~km} \mathrm{~s}^{-1}$ and $R V>+300 \mathrm{~km} \mathrm{~s}^{-1}$, and they are like the continuum not obscured. A better sampling of the $R V$-space and the behaviour for days with high velocity absorption components are discussed in the following sections.

The two narrow line features in the vicinity of the strong Balmer lines $\left(-300 \mathrm{~km} \mathrm{~s}^{-1}<R V<+300 \mathrm{~km} \mathrm{~s}^{-1}\right)$ are probably affected by absorption and radiative transfer effects in the $\mathrm{H}$ I line wings.
Comparison with XX Oph. The symbiotic-like star XX Oph has a very similar line spectrum as MWC 560 (see e.g., Kolev \& Tomov 1993; Michalitsianos et al. 1991). Therefore, it is very useful to compare the line fluxes for these two stars and investigate which emission lines in MWC 560 are supressed by absorption. For this purpose we compare the MWC 560 spectrum from day 153 with an Echelle spectrum of XX Oph taken by Toma Tomov with the $1.82 \mathrm{~m}$ telescope of the Asiago Astrophysical Observatory during the night of March 22, 1995. The spectral region around $\mathrm{H} \beta$ is plotted for XX Oph and MWC 560 in the upper panel of Fig. 16. It is clearly visible that the narrow lines in the $\mathrm{H} \beta$ absorption trough of MWC 560, i.e. the lines $i, j, k$ and $l$ in Fig. 16, are significantly weaker than the corresponding lines in XX Oph. Table 8 gives line ratios $I_{153} / I_{\mathrm{XXOph}}$ for the stronger lines in the $\mathrm{H} \beta$ and $\mathrm{H} \gamma$ regions, whereby $I_{153}$ (for MWC 560) and $I_{\mathrm{XXOph}}$ are normalized line fluxes $f_{\ell}$. According to this table the narrow lines in the $R V$-range $-1300 \mathrm{~km} \mathrm{~s}^{-1}$ to $-600 \mathrm{~km} \mathrm{~s}^{-1}$ of $\mathrm{H} \beta$ or $\mathrm{H} \gamma$ are strongly attenuated by about a factor 5-10 in MWC 560 compared to the relative line strengths measured for XX Oph. No attenuation effect seems to be present for other $R V$-regions. Thereby it must be noted that this method yields no information about attenuation of narrow lines in MWC 560 for the $R V$-range from about $-500 \mathrm{~km} \mathrm{~s}^{-1}$ to $+290 \mathrm{~km} \mathrm{~s}^{-1}$ because of the strong H I emission in both stars and the P Cygni-type absorption in XX Oph.

Thus, the $I_{153} / I_{\mathrm{XxOph}}$ line ratios in Table 8 agree with the finding made above based on atomic branching ratios. But in the comparison with XX Oph more lines could be included in the analysis giving a better coverage of the $R V$-space. This defines well the $R V$-edge of the jet absorption in front of the narrow line region at $R V_{\text {edge }} \cong$ $-1300 \mathrm{~km} \mathrm{~s}^{-1}$ for day 153 . This value agrees with the jet absorption edge for the continuum.

Temporal variations. In the previous two paragraphs the occultation of narrow lines by the persistent main jet component $\left(R V\right.$-range $-1300 \mathrm{~km} \mathrm{~s}^{-1}$ to $\left.-600 \mathrm{~km} \mathrm{~s}^{-1}\right)$ was considered. We now concentrate on occultation of narrow emissions by the variable high velocity components of the jet. This effect can be well studied for the numerous strong lines located on the blue side of the $\mathrm{H} \gamma$-region. Figure 16 compares the spectra of day 153 and day 194 representing the two extreme cases without and with a very strong high velocity component. It is visible that the lines between $4308 \AA$ and $4325 \AA$ (lines $e, f, g, h, i$ and $k$ in the lower panel of Fig. 16) are weaker in the extended absorption trough of day 194 compared to day 153 . Table 8 gives the measured line ratios $I_{194} / I_{153}$ for the strong lines in the $\mathrm{H} \beta$ and $\mathrm{H} \gamma$ regions.

From Table 8 follows that the lines in the $R V$-range $-1900 \mathrm{~km} \mathrm{~s}^{-1}$ to $-1300 \mathrm{~km} \mathrm{~s}^{-1}$ are weakened for day 194 relative to day 153 . The line ratios $I_{194} / I_{153}$ are for this $R V$-range only $0.55 \pm 0.10$ times the value measured for lines with $R V<-2000 \mathrm{~km} \mathrm{~s}^{-1}$ or $>+300 \mathrm{~km} \mathrm{~s}^{-1}$. 
Table 8. Fluxes for narrow lines in and near the $\mathrm{H} \beta$ and $\mathrm{H} \gamma$ absorption troughs. Letters on the left refer to Fig. 16. $R V_{\text {obs }}$ is the barycentric radial velocity of the line in MWC 560. $I_{153}$, $I_{194}$ and $I_{\text {XXOph }}$ are normalized line fluxes $f_{\ell}$ for day 153 and day 194 for MWC 560 and the comparison star XX Oph, respectively. Bold and slanted values are given for particularly low (bold numbers) and high (slanted numbers) line ratios caused by the attenuation from the $\mathrm{H} \beta$ or $\mathrm{H} \gamma$ jet absorptions in MWC 560.

\begin{tabular}{|c|c|c|c|c|c|}
\hline & $\lambda_{\text {lab }} /$ ident & $R V_{\mathrm{obs}}$ & $I_{153}$ & $\frac{I_{194}}{I_{153}}$ & $\frac{I_{153}}{I_{\mathrm{XXOph}}}$ \\
\hline \multicolumn{6}{|c|}{$\mathrm{H} \beta$-region } \\
\hline & 4798.53 Ti II 17 & -3837 & 0.207 & 0.97 & 0.83 \\
\hline $\mathrm{a}$ & 4805.09 Ti II 92 & -3432 & 0.387 & 0.98 & 1.05 \\
\hline $\mathrm{b}$ & 4812.34 Cr II 30 & -2985 & 0.102 & 0.78 & 0.43 \\
\hline $\mathrm{c}$ & 4814.55 [Fe II] 20F & -2849 & 0.108 & 0.87 & 0.64 \\
\hline d & 4823.31 Y II 22 & -2308 & 0.129 & 0.86 & \multirow{2}{*}{\} 0.86} \\
\hline e & 4824.14 Cr II 30 & -2258 & 0.413 & 0.78 & \\
\hline $\mathrm{f}$ & 4825.72 Fe II 30 & -2164 & 0.073 & 1.1 & 0.43 \\
\hline $\mathrm{g}$ & 4833.21 Fe II 30 & -1699 & 0.064 & 0.44 & 0.36 \\
\hline $\mathrm{h}$ & 4836.22 Cr II 30 & -1512 & 0.145 & 0.39 & 0.56 \\
\hline $\mathrm{i}$ & $4839.9 ?$ & -1281 & $<0.030$ & & $<0.19$ \\
\hline $\mathrm{j}$ & $4846.4 ?$ & -884 & $<0.010$ & & $<0.10$ \\
\hline $\mathrm{k}$ & 4848.24 Cr II 30 & -767 & 0.073 & 1.2 & 0.15 \\
\hline 1 & 4849.18 Ti II 29 & -709 & 0.016 & 1.1 & 0.06 \\
\hline $\mathrm{m}$ & 4865.61 Ti II 29 & +298 & 0.121 & 0.55 & 0.61 \\
\hline $\mathrm{n}$ & 4871.27 Fe II 25 & +649 & 0.122 & 0.48 & 0.49 \\
\hline $\mathrm{o}$ & 4874.01 Ti II 114 & +818 & 0.284 & 0.75 & 0.86 \\
\hline \multirow[t]{4}{*}{$\mathrm{p}$} & 4876.4 Ti II $30^{a}$ & +967 & 0.299 & 0.98 & 0.75 \\
\hline & 4883.68 Y II 22 & +1414 & 0.221 & 0.85 & 1.30 \\
\hline & 4884.60 Cr II 30 & +1471 & & 0.69 & 0.44 \\
\hline & $4889.62[\mathrm{Fe} \mathrm{II}] 4 \mathrm{~F}$ & +1780 & 0.080 & 0.95 & 0.62 \\
\hline \multicolumn{6}{|c|}{$\mathrm{H} \gamma$-region } \\
\hline & 4290.22 Ti II 41 & -3822 & 0.686 & 0.90 & 1.11 \\
\hline & 4294.10 Ti II 20 & -3167 & 0.452 & 0.84 & 0.96 \\
\hline & 4296.57 Fe II 28 & -2996 & 0.474 & 0.87 & 0.62 \\
\hline $\mathrm{a}$ & 4300.06 Ti II 41 & -2755 & 0.858 & 0.91 & 1.30 \\
\hline $\mathrm{b}$ & 4301.93 Ti II 41 & -2627 & 0.636 & 0.86 & 1.23 \\
\hline $\mathrm{c}$ & 4303.17 Fe II 27 & -2540 & 0.745 & 0.83 & 1.00 \\
\hline $\mathrm{d}$ & 4307.90 Ti II 41 & -2216 & 0.596 & 0.81 & 1.49 \\
\hline e & 4312.86 Ti II 41 & -1871 & 0.584 & 0.53 & 1.22 \\
\hline $\mathrm{f}$ & $4314.2 \mathrm{Sc}$ II $/ \mathrm{Fe}$ II $^{a}$ & -1782 & 0.467 & 0.48 & 0.97 \\
\hline $\mathrm{g}$ & 4314.98 Ti II 41 & -1726 & 0.427 & 0.45 & 1.47 \\
\hline $\mathrm{h}$ & 4316.81 Ti II 94 & -1600 & 0.166 & 0.46 & \multirow{2}{*}{\} 1.48} \\
\hline $\mathrm{i}$ & 4317.32 Zr II 40 & -1565 & 0.071 & 0.52 & \\
\hline $\mathrm{j}$ & $4319.62[\mathrm{Fe} \mathrm{II}] 21 \mathrm{~F}$ & -1404 & 0.044 & 0.77 & $b$ \\
\hline $\mathrm{k}$ & 4320.8 Sc II $/ \mathrm{Ti} \mathrm{II}^{a}$ & -1324 & 0.300 & 0.51 & 0.97 \\
\hline 1 & 4325.01 Sc II 15 & -1032 & 0.069 & 1.1 & 0.30 \\
\hline $\mathrm{m}$ & 4325.76 Fe I 42 & -981 & 0.036 & 1.2 & 0.15 \\
\hline $\mathrm{n}$ & 4330.26 Ti II 94 & -668 & 0.021 & 2.5 & \multirow[t]{2}{*}{ \}o.12 } \\
\hline $\mathrm{o}$ & 4330.71 Ti II 41 & -639 & 0.041 & 2.2 & \\
\hline $\mathrm{p}$ & 4337.92 Ti II 20 & -138 & 0.115 & 0.96 & $c$ \\
\hline \multirow[t]{3}{*}{$q$} & 4344.29 Ti II 20 & +299 & 0.231 & 0.58 & 0.75 \\
\hline & 4351.76 Fe II 27 & +816 & 1.138 & 0.80 & 0.98 \\
\hline & 4367.66 Ti II 104 & +1914 & 0.328 & 1.00 & 0.84 \\
\hline
\end{tabular}

a: Blended lines; 4876.40/.48 Ti Iı $30+$ Ti Iı 30; 4314.08/.29 Sc II 15 + Fe II 32; 4320.74/.95 Sc II $15+$ Ti II 41. $b$ : Too weak to be measured in our XX Oph spectrum. $c$ : Affected in XX Oph by the P Cygni-absorption of $\mathrm{H} \gamma$.

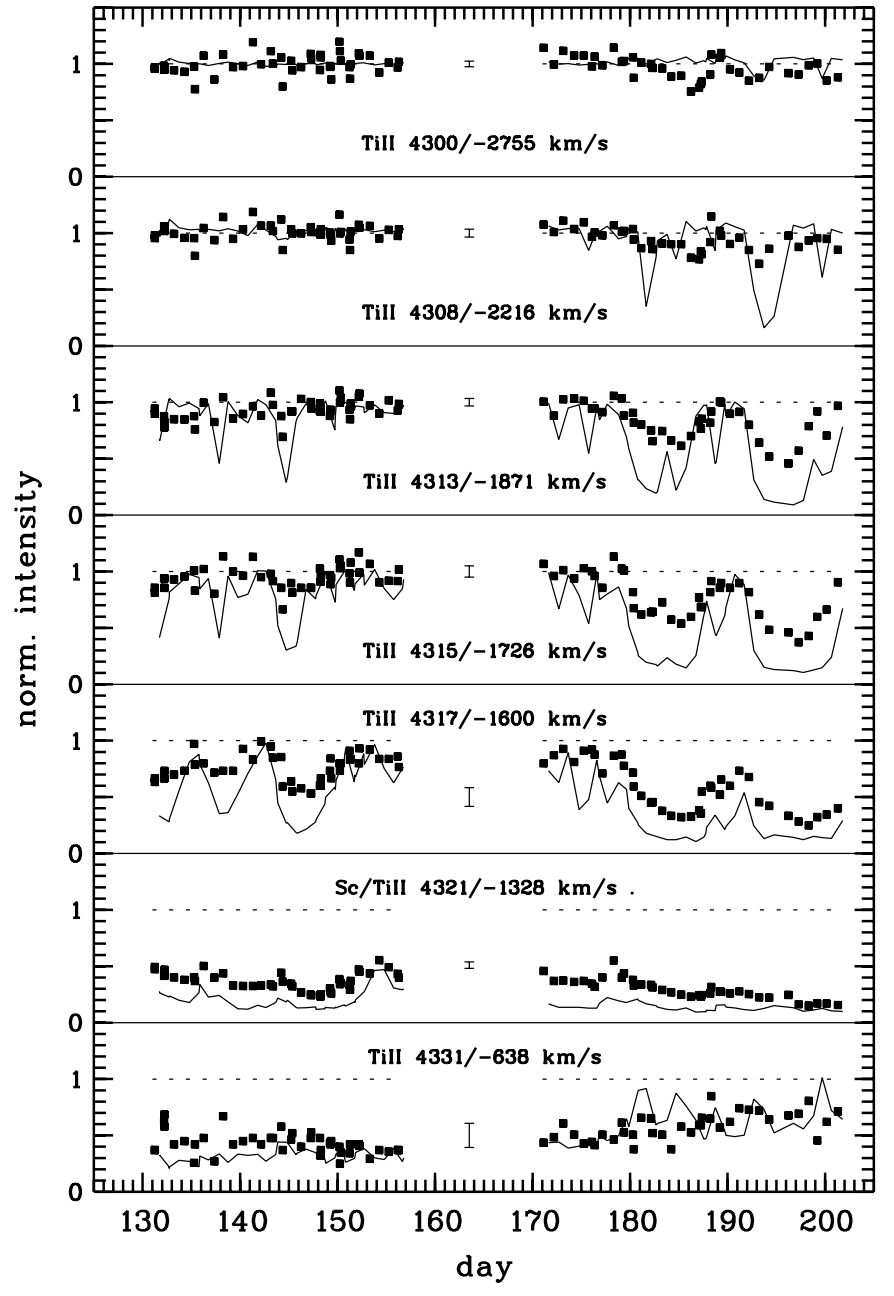

Fig. 17. Obscuration of the narrow emission lines by the $\mathrm{H} \gamma$ jet absorption. Line fluxes (filled squares) are compared to the continuum variations (solid lines) measured for the $\mathrm{H} \beta$-region at the same $R V(\mathrm{H} \beta)$ as the $R V(\mathrm{H} \gamma)$ for the narrow line. Typical error bars for individual line measurements are given in the center of each panel. The flux scale and the dotted line for $f=1$ refer to the normalized continuum intensity. The fluxes for the narrow lines were scaled in order to match $f=1$ for phases where no obscuration seems to be present. For Sc/Ti II $\lambda 4321$ and Ti II $\lambda 4331$ the scaling is arbitrary assuming just some degree of obscuration.

A noticable exception is the [Fe II] $\lambda 4319.62$ line flux, which stays practically constant. This supports the previous finding that forbidden lines originate predominantly from a different (larger) region than the allowed lines (Sect. 6.1). In addition it is found that the lines " $n$ " and " $O$ " in the lower panel of Fig. 16 near $R V=$ $-650 \mathrm{~km} \mathrm{~s}^{-1}$ are significantly stronger for day $194 \mathrm{com}-$ pared to day 153. Thus also the narrow lines display the anti-correlation between high and low velocity absorption depths described for the continuum in Sect. 4.3.

The temporal flux changes for various narrow lines in the $\mathrm{H} \gamma$ region is plotted in Fig. 17. There the measured line fluxes are given together with the continuum variations at the same radial velocity measured for the $\mathrm{H} \beta$ jet 
absorption. This figure illustrates that the strong line flux changes coincide with strong continuum flux changes. However, the decrease in line flux is less than the continuum flux decrease. The attenuation level for the narrow emission lines indicates that the transient high velocity gas obscures not more than about half of the narrow line emission region. Further, Fig. 17 shows that short lived high velocity components (or the highest velocity parts of strong components) clearly present in the continuum are hardly or not seen in the line fluxes. Examples are in Fig. 17 the components at $R V=-2216 \mathrm{~km} \mathrm{~s}^{-1}$ for days 180 and $193 / 194$ or at $-1726 \mathrm{~km} \mathrm{~s}^{-1}$ for days $144 / 145,175$, 188 and more. This suggests that these absorptions result from geometrically small structures, small compared to the characteristic size of the Fe II-emitting narrow line region.

\subsection{Jet structure and narrow line region}

Because the allowed emission lines from neutral and ionized heavy elements vary in strength like the hot continuum they must originate close to the accreting component. Thereby the narrow lines from neutral species and singly ionized elements, like Ca II and Ti II require a gas with low ionization $N(\mathrm{HII}) / N(\mathrm{HI}) \ll 1$, which must therefore be well shielded from the ionizing radiation of the accreting component. In addition, these lines have a radial velocity close to the systemic radial velocity and are located behind a fast moving outflow approching us. All these properties suggest that the narrow emission line region can be associated with the (probably partly irradiated) layers of the cool, neutral part of the accretion disk seen face on.

The jet absorption components obscure this (variable) narrow line region in different ways.

Major high velocity components are able to occult about half of the narrow line region. However, the initial highest velocity continuum absorptions produce hardly a flux weakening for the narrow lines. Thus these transient high velocity absorptions are small and then grow with time as they decelerate to dimensions of roughly half the size of the narrow line region. The short lived minor high velocity components can not be clearly recognized in the fluxes of the narrow lines. Thus they cover less than about $20 \%$ of the narrow line region.

For radial velocities in the intermediate range $R V \approx$ -1300 to $-600 \mathrm{kms}^{-1}$ the narrow lines are weakened due to the outflowing gas to a residual relative flux of about 0.2 or even less. Thus the intermediate velocity gas covers practically the entire (variable) narrow line region. Thereby the lines around $R V \approx-700 \mathrm{~km} \mathrm{~s}^{-1}$ vary like the shallow low velocity components associated previously with the initial acceleration region. Around $R V \approx-1100 \mathrm{~km} \mathrm{~s}^{-1}$ close to the jet terminal velocity $v_{\infty}$ the occultation of practically the entire narrow line region is probably caused by the jet outflow at larger distances from the source.

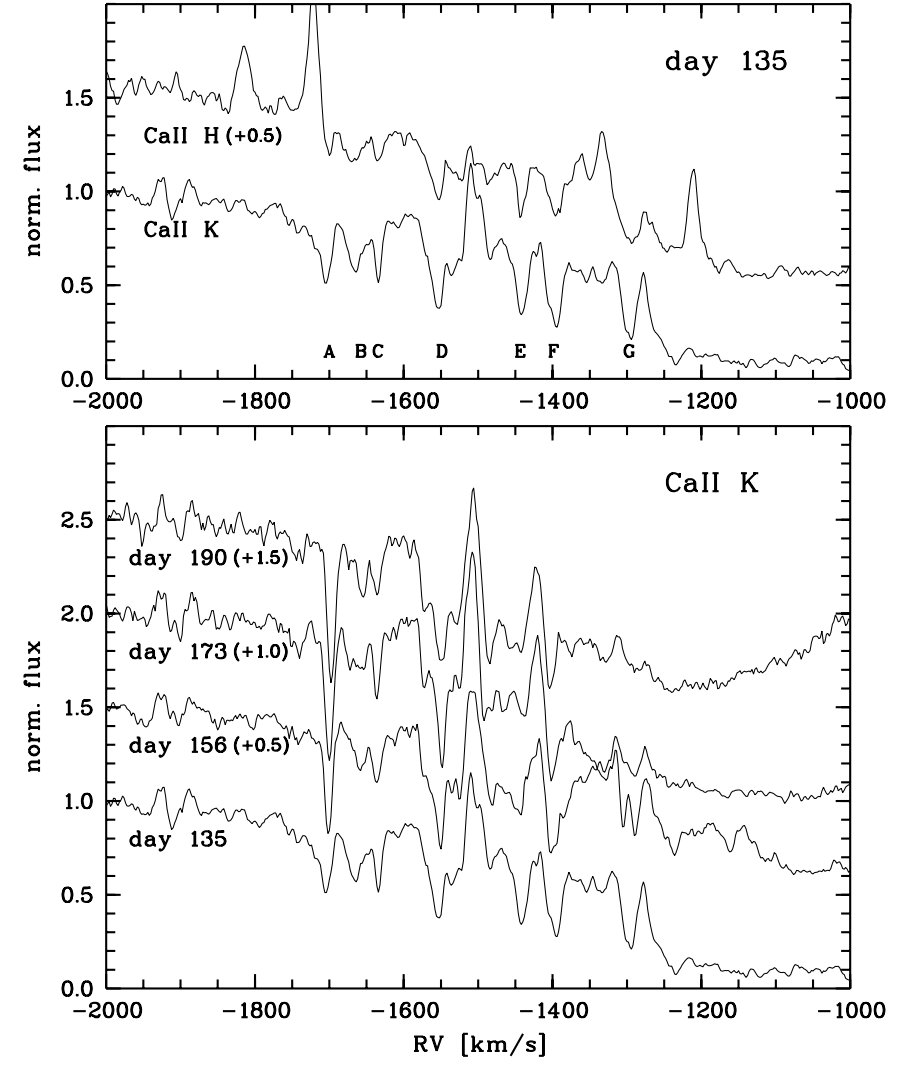

Fig. 18. Fast, narrow Ca II absorptions in MWC 560. The upper panel shows a comparison between the absorption components in the $\mathrm{H}$ and $\mathrm{K}$ line and the lower panel the time evolution for Ca II K. The barycentric radial velocities are given relative to the rest wavelengths $3933.66 \AA$ for Ca II K and $3968.47 \AA$ for Ca II H.

\section{Fast, narrow Ca II absorptions}

\subsection{Spectroscopic properties}

Both resonance lines of Ca II show several blue shifted narrow absorption components (Fig. 18; see also Fig. 8b). The radial velocities of these narrow absorptions lie in the range $-1750 \mathrm{~km} \mathrm{~s}^{-1}$ to $-1250 \mathrm{~km} \mathrm{~s}^{-1}$. The narrow absorptions are of course only visible when the strongly variable broad absorption troughs extend not too far to the blue or are shallow as for day 193. In Fig. 18 the seven strongest absorption lines seen for day 135 are identified. Despite some emission lines it is visible that all these components occur for both CaII resonance transitions. Line parameters for the absorption components $\mathrm{A}$ to $\mathrm{F}$ are given in Table 9 .

The typical equivalent widths of the identified narrow CaII absorptions are about $0.05-0.20 \AA$. The equivalent widths are measured with respect to a pseudo-continuum level defined by (relatively) line free sections, e.g., at $R V=-1600 \mathrm{~km} \mathrm{~s}^{-1}$ or $-1350 \mathrm{~km} \mathrm{~s}^{-1}$. The decrease of the continuum towards the red is caused by a high velocity absorption wing from the broad absorption troughs.

For the widths of the narrow absorptions we measure $10-30 \mathrm{kms}^{-1}(F W H M)$. But several of the features are 
again composed of subfeatures, e.g., F and G for day 135. Absorptions with "clean" Gaussian profiles (e.g., feature A for days $156,173,190$, or feature $\mathrm{C}$, etc.) have practically always a width of only $10-15 \mathrm{~km} \mathrm{~s}^{-1}(0.13-0.18 \AA)$.

The lower panel of Fig. 18 demonstrates that the absorptions remain rather stable on time scales of months. Therefore the narrow lines can also be seen in the mean spectrum plotted in Fig. 1 near $3915 \AA$ and $3950 \AA$. Observed changes over 55 days are a $50 \%$ increase of the strengths for feature A, the emergence of a new component in the blue wing of feature D, a substantial weakening of feature $\mathrm{E}$, or a temporary splitting of feature $\mathrm{G}$ into two components around day 156. All these changes are not only seen in the CaII K transition as shown in Fig. 18, but also in CaII H. From this we conclude that the typical lifetime of the blue shifted, narrow absorption lines is of the order of a month or longer.

Radial velocity changes up to $|\Delta R V|=10 \mathrm{~km} \mathrm{~s}^{-1}$ are measured for the line centers of the narrow absorptions (Table 9). Thereby it must be carefully distinguished whether we see a true line shift or just a change in relative strength of subcomponents within a given feature. The latter case seems to happen for absorption features B and F. However, the line profile evolution of feature $\mathrm{A}$ and the strong central component of feature D indicate a real shift in radial velocity towards lower outflow velocity. This evolution could therefore be caused by a deceleration of the corresponding outflow components.

Narrow absorption features are not visible redward of the broad absoption troughs $R V \gtrsim-1000 \mathrm{kms}^{-1}$. Due to the broad absorption no continuum flux is usually visible from about $R V=-1250 \mathrm{~km} \mathrm{~s}^{-1}$ to $-1000 \mathrm{~km} \mathrm{~s}^{-1}$. An exception are the spectra around day 193 where the variable absorption troughs are extremely broad but shallow (Fig. 2). These spectra show that narrow absorptions are absent for $R V>-1250 \mathrm{~km} \mathrm{~s}^{-1}$.

We have searched for corresponding narrow absorptions (i.e. same $R V$ as for $\mathrm{Ca}$ II) in the strong $\mathrm{H}$ I Balmer transitions and for $\mathrm{NaI}$ with negative result. An upper limit for the equivalent widths for such narrow absorptions is about $0.03 \AA$ in $\mathrm{H} \beta$, NaI or $\mathrm{H} \alpha$.

\subsection{Physical parameters of the fast, narrow absorptions}

The fast, narrow absorptions are certainly closely linked to the jet outflow in MWC 560, because the radial velocity of the narrow components is comparable to the $R V$ of the jet outflow. Further we know that the jet is directed towards us, so that an absorption in the continuum spectrum of the jet source must be due to gas located close to the jet outflow.

Column densities for the narrow high velocity absorption components are $N(\mathrm{Ca}$ II $) \approx 1 \times 10^{12} \mathrm{~cm}^{-2}$ calculated for an $E W$ of $0.1 \AA$ (see Sect. 3.1). Adopting solar abundances and assuming that CaII is the dominant ionization stage of calcium yields a hydrogen column density of
Table 9. Parameters for the Ca II narrow absorption components $\mathrm{A}$ to $\mathrm{F}$ identified in Fig. 18.

\begin{tabular}{lccccc}
\hline & \multicolumn{3}{c}{ day 135} & \multicolumn{2}{c}{ day 190} \\
& $R V$ & \multicolumn{2}{c}{$E W[\AA]$} & $R V$ & $E W[\AA]$ \\
& {$\left[\mathrm{km} \mathrm{s}^{-1}\right]$} & Ca II K & Ca II H & {$\left[\mathrm{km} \mathrm{s}^{-1}\right]$} & Ca II K \\
\hline $\mathrm{A}$ & -1705 & 0.10 & blend & -1697 & 0.15 \\
$\mathrm{~B}$ & -1665 & 0.10 & 0.08 & -1655 & 0.05 \\
$\mathrm{C}$ & -1635 & 0.06 & 0.04 & -1637 & 0.04 \\
$\mathrm{D}$ & -1554 & 0.14 & 0.09 & -1550 & 0.25 \\
$\mathrm{E}$ & -1441 & 0.12 & 0.06 & absent & \\
$\mathrm{F}$ & -1396 & 0.18 & 0.09 & -1401 & 0.10 \\
$\mathrm{G}$ & -1296 & 0.16 & $a$ & $a$ & $a$ \\
error & \pm 1 & \pm 0.02 & \pm 0.02 & \pm 1 & \pm 0.02 \\
\hline
\end{tabular}

$a$ : Not visible due to the broad absorption trough.

$N(\mathrm{H})=5 \times 10^{17} \mathrm{~cm}^{-2}$ for a typical narrow absorption component.

The fact that there are no similar absorptions visible for the Balmer lines suggests a low temperature of roughly $T_{\mathrm{e}} \approx 10000 \mathrm{~K}$ for the absorbing gas. This follows from the expected $n=2$ level population of H I (Boltzmann-factor) and ionization degree of calcium and hydrogen in collisional (coronal) equilibrium (Arnaud \& Rothenflug 1985).

The observations suggest a life time of these components of one month or longer during which they travel about $30 \mathrm{AU}$ (for $R V=-1700 \mathrm{~km} \mathrm{~s}^{-1}$ ) further away from the source. Thus their mean distance to the source is of the order $10-100 \mathrm{AU}$ or larger. This fast moving gas will produce shocks in the expectedly very heterogeneous circumstellar environment of MWC 560. Thus, the fact that we see cool gas requires a short cooling time $t_{\mathrm{c}}[\mathrm{s}] \approx 6 \times 10^{11} / N_{\mathrm{e}}$ (e.g., Spitzer 1978) compared to the life time of an absorption component. Thus, the density of the cool gas is at least of the order $N_{\mathrm{e}} \gtrsim 10^{6} \mathrm{~cm}^{-3}$ or larger to allow cooling of the shocked gas within a few days. This yields an extension of less than $\Delta d \lesssim 10^{11} \mathrm{~cm}$ along the line of sight for an absorbing Ca II gas component. Gas clumps of this small size and high speed are likely to be immediately dispersed. However, thin radiative shock fronts generated by the jet could survive for a long time.

\section{Interpretation and discussion}

In the previous sections we have determined from our spectroscopic data model free parameters like time scales, radial velocities, column densities or covering factors. Considering further that we see a fast outflow from the hot component in a symbiotic binary yields already a rough velocity structure for the outflowing gas. Thus it is possible to construct a schematic geometric model for the gas outflow in the MWC 560 system without knowing accurate system parameters. This basic outflow geometry will be illustrated in the following section. 
In order to estimate more accuratly the geometric dimensions and physical parameters of the jet outflow we have to adopt system parameters like distance, binary separation, orbital inclination and more. For this we review existing estimates in the literature and make our own determinations if necessary.

\subsection{Schematic outflow geometry}

Figure 19 gives a schematic illustration of the jet geometry showing the location of the emission components and the lines of sights (dotted) along which the outflowing gas produces the different absorption components.

Emission components. There are three different emission components which are absorbed by the jet outflow:

- the variable hot continuum source;

- the variable narrow line region emitting allowed lines of mainly singly ionized heavy elements;

- the red giant.

The variable hot continuum is due to emission from the accreting object and the inner accretion disk. However it must be suspected that the hot source geometry is complicated and not well known yet. In particular it must be considered that irradiation and reflection effects between central object and accretion disk may cause a substantial continuum emission from a more extended region than just the immediate suroundings of disk boundary layer and the accreting star.

The Fe II emission line region is closely associated with the hot continuum source, because the line strengths vary in step with the continuum variations. As the narrow lines are at the systemic radial velocities it can be assumed that they originate in the accretion disk, probably in the irradiated upper layers (chromosphere) of the neutral disk. Varying irradiation can change the ionization fraction of the partly ionized emission region and cause immediate flux changes in the emission lines in step with the continuum radiation. Based on these arguments, a disk structure seems to be the only viable geometry for the Fe II emission line region. However, the extent and the radial flux distribution of the line emission is unclear. In addition there is some emission of forbidden lines, like [O I] and [Fe II], from an extended low density region. It is difficult to constrain the geometric location of this region. Because the lines are narrow and at the systemic $R V$ they could originate in the cool outer accretion disk or the outer atmosphere of the cool giant.

The late spectral type M5.5 III of the cool giant indicates a very extended photospheric emission $R \approx 140 R_{\odot}$ located beside the accretion disk and hot continuum source in the binary system seen practically pole on.

Structure of the jet outflow. Based on the structure and variability of the jet absorptions discussed in detail in

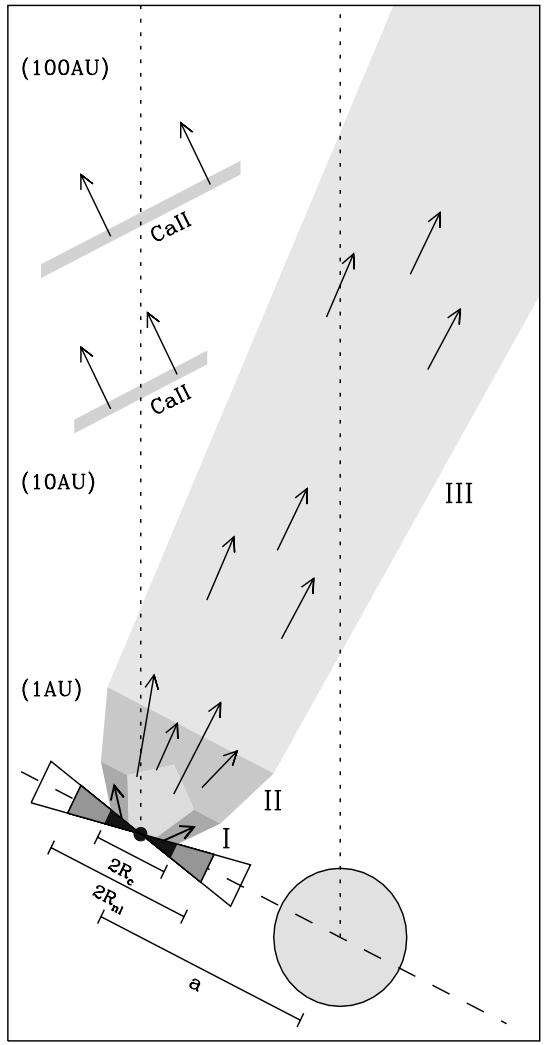

Fig. 19. Schematic geometry of the MWC 560 outflow and the location of the line of sights (dotted lines) to the continuum source and to the red giant. The outflow is divided into three jet regions I, II and III according to the properties of the corresponding absorption components. For illustration purposes the different components are not drawn to scale (see approximate distances along the jet indicated on the left) and the inclination of the jet (or orbital plane) is too large. In particular the line of sight from the accreting object should pass for a longer distance through jet region III. Geometric dimensions for the continuum source $2 R_{\mathrm{c}}$, the narrow line region $2 R_{\mathrm{nl}}$ and the binary separation $a$ are indicated.

Sect. 4.6 three different outflow regions along the jet axis are found having distinct radial velocity properties:

I: The initial acceleration region characterized by low (negative) radial velocities $R V \gtrsim-600 \mathrm{~km} \mathrm{~s}^{-1}$ covers only partly the continuum source. This region covers also partly the $\mathrm{H}$ I emission line region and is therefore mainly located beside the central accreting object;

II: A region in front of the hot continuum source showing repeatedly transient high velocity components $R V \approx-1500$ to $-2500 \mathrm{~km} \mathrm{~s}^{-1}$ with life times of one to a few days. Major high velocity components reach maximum velocity within one day and display thereafter a continous deceleration back to at most $-1500 \mathrm{~km} \mathrm{~s}^{-1}$. The appearence of high velocity components is anti-correlated and therefore closely related to the low velocity absorption of the initial acceleration region I. The life time of the high velocity components 
suggests that this outflow region extends to about 1-3 AU along the jet axis;

III: A third region with a persistent flow at an intermediate radial velocity in the range $\approx-900$ to $-1300 \mathrm{~km} \mathrm{~s}^{-1}$. The life time of individual components suggests an extension along the jet axis of up to $10 \mathrm{AU}$ or more. At the beginning of our compaign this steady flow covers also the red giant with two $R V$ components at about -1200 and $-1100 \mathrm{~km} \mathrm{~s}^{-1}$. The obscuration of the red giant by jet gas disappears during the following two months as expected if a collimated polar jet outflow from a source in a binary covers the companion during the (inferior) conjunction phase.

This separation into three regions is based on the radial velocity structure of the jet absorptions. As noticed in our description of absorptions from different ions, like $\mathrm{NaI}$, Fe II, HI and HeI the jet outflow displays also a stratification in gas excitation and ionization degree. However we defer the investigation of these properties to a future paper.

The three radial velocity components cover also at least partly the narrow line emission region as worked out in Sect. 6.

The low velocity region I causes a measurable weakening in the $R V$ range -600 to $-100 \mathrm{~km} \mathrm{~s}^{-1}$ of the Balmer lines but does not completely extinguish the narrow lines or the $\mathrm{H}$ I blue line wing emission. Thus a substantial fraction $(\gtrsim 30 \%)$ of the extended emission line region is covered by low velocity gas. At the same time only half or less of the much smaller central continuum source is behind this low velocity component. This is strong evidence that the initial outflow acceleration occurs above the accretion disk. Acceleration of gas could also occur close to the compact accreting object without producing low velocity absorption components. If the outflow rate from this region is large enough then the wind can be optically thick due to electron scattering, so that only the fast moving gas at radii $r>R_{\tau=1}$ is visible (see also Sect. 8.2). A more detailed analysis of the absorption components is required to support or refute such an outflow from the central region.

Major high velocity components from region II cover fully the continuum source but only about half of the narrow emission line region. Thereby, the size of a given component is initially small and then grows as its velocity is decelerated. Short lived ( $<2$ days), minor high velocity components absorb often only about half of the continuum radiation and cause no significant weakening $(<20 \%)$ of the narrow line emission. This indicates that the high velocity components have different geometrical dimensions perpendicular to the jet axis ranging from about the size of the continuum source for minor component to about half the size of the narrow emission line region for major components.

Gas from the more remote outflow region III covers fully the continuum source and about $80 \%$ or more of the narrow emission line region. In addition the outflow at large distance from the jet source is also capable to cover at least $75 \%$ of the red giant companion. Thus the jet radius must reach at larger distances from the source the dimensions of the narrow line region and the red giant companion.

From this discussion it can be concluded that the jet radius in MWC 560 increases with distance from the jet source.

The Ca II shock fronts. Figure 19 indicates also schematically the position of the narrow, fast Ca II absorption components. As discussed in in Sect. 7 they can be associated with radiative shocks generated by the jet outflow.

A very intriguing property of the narrow absorptions is that their $R V$ is larger than the terminal $R V$ of the jet as derived from the $\mathrm{H} \alpha$ jet absorption in front of the red giant. Because shock fronts move slower than the pre-shock gas, and since only the transient high velocity jet components have velocities in excess of the narrow Ca II absorptions, it must be concluded that they are responsible for these structures. Thus the cool Ca II shock fronts are most likely generated by the shocks due to the collision of the transient high velocity jet components with previously ejected slower gas in the jet.

The generation of shocks is expected for variable jet outflows, where the amplitude of the velocity variations exceeds the sound speed. If the pressure in the generated shocks internal to the jet is high enough then the gas is forced out of the jet in lateral direction producing fast moving shock fronts in the jet cocoon. This was shown by numerical simulation (e.g., Stone \& Norman 1993) although not for jet parameters similiar to MWC 560. Thus shocks are certainly present in region II in MWC 560, where transient components have a velocity which is up to about $1000 \mathrm{~km} \mathrm{~s}^{-1}$ higher than the mean jet velocity. Further the covering factor of the high velocity components increases as they are decelerated indicating a lateral expansion of the shocked gas. This may force fast moving jet gas into the so-called jet cocoon producing shock regions. Whether, such shocks can cool and form Ca II absorption features with the temperature, column density, life time and velocity as observed in MWC 560 remains to be investigated. However, the number of observed CaII features can be explained with this scenario. If the Ca II shock fronts are generated by the high velocity components occuring on a time scale of about a week, then their inferred lifetime of about a couple of months yields the observed number of Ca II features of about 5 to 10 .

\subsection{Parameters for MWC 560 binary system}

Deriving system parameters for MWC 560 has often to rely on indirect methods and assumptions. Therefore the uncertainties are hard to quantify and the derived results have to be considered cautiously. In Table 10 the obtained parameters for MWC 560 are summarized and detailed discussions are given for each value in the following paragraphs. We are aware that many of these estimates 
can be considerably improved with detailed investigations which are, however, beyond the scope of this paper. Thus Table 10 is a compilation of parameters which will hopefully be strongly improved in the near future.

Orbital period and binary separation. Unfortunately the orbital period for the MWC 560 binary system is not known. An estimate can be made from a relation of Mürset \& Schmid (1999), which describes for symbiotic systems the shortest orbital period to be expected for a given spectral type for the red giant. According to this the expected period for MWC 560, which contains a M5.5 giant, is 840 days or longer.

As discussed in Sect. 5 the obscuration of the red giant by the jet as seen in the $\mathrm{H} \alpha$ absorption is probably caused by a periodic eclipse effect locked to the binary orbit. Our observations cover only the egress phase of this obscuration which lasts about 2 months. We are not aware of other observations of the $\mathrm{H} \alpha$ jet absorption structure taken earlier in 1998 which could further constrain the full duration of this event. However, a lower limit on the full eclipse phase is 4 months assuming that the duration of the ingress phase is comparable to the egress phase. A similar jet absorption in the spectrum of the red giant was seen for the He I $\lambda 10831$ line in April 1990, and less prominent in January 1991, by Meier et al. (1996). This may be interpreted as obscuration phase lasting about 10 months or longer. If both are eclipse effects due to the binary orbit of MWC 560 then the orbital period is $P \approx 8.5$ years or an integer fraction of this. Thus, the other possibilities above the lower limit of Mürset \& Schmid (1999) are the periods $P=4.25$ or 2.83 years. Thereby the long duration of the obscuration phase favours long orbital periods. Therefore we consider in the following only $P=4.25$ and 8.5 years.

For an estimate on the binary separation we assume stellar masses of $0.5 M_{\odot}$ and $1.5 M_{\odot}$ for the accreting object and the red giant respectively. Such masses are typical for symbiotic systems (e.g., Mürset et al. 2000). This yields for circular orbits a binary separation of $a=5.2$ AU for a period of $P=8.5 \mathrm{yr}$, or $3.3 \mathrm{AU}$ for $P=4.25 \mathrm{yr}$.

Jet and orbital inclination. It is assumed that the jet axis is perpendicular to the orbital plane. This assumption is made for simplicity and because there is currently no evidence that the jet outflow or the accretion disk in MWC 560 are tilted with respect to the frame defined by the binary orbit.

The upper limit on the inclination can be obtained from limits on orbital radial velocity variations. With the parameters adopted above the orbital velocities of the two stellar components with respect to the center of mass are $v_{\mathrm{rg}}=4.6 \mathrm{~km} \mathrm{~s}^{-1}$ for the red giant and $v_{\mathrm{hc}}=13.8 \mathrm{~km} \mathrm{~s}^{-1}$ for the hot component assuming a period of $P=8.5 \mathrm{yr}$. The corresponding values for $P=4.25 \mathrm{yr}$ are $v_{\mathrm{rg}}=$ $5.8 \mathrm{~km} \mathrm{~s}^{-1}$ and $v_{\mathrm{hc}}=17.4 \mathrm{~km} \mathrm{~s}^{-1}$.

Unpublished high resolution spectroscopy of red giant absorption lines from MWC 560 taken with the coude echelle spectrograph (CES) at the ESO $1.4 \mathrm{~m}$ CAT telescope by the Zürich group (see e.g., Schild et al. 2001) yields a mean radial velocity of $36.7 \mathrm{~km} \mathrm{~s}^{-1}$ with a scatter of $\sigma=1.1 \mathrm{~km} \mathrm{~s}^{-1}$ for 16 measurements taken between April 1992 and December 1997. Our FEROS spectra provide daily radial velocities for the red giant. The measurements show that there exist $R V$ variations of about $0.4 \mathrm{~km} \mathrm{~s}^{-1}$ on time scales of a few days. Further, the mean velocity determined for our campaign is between about $37.8 \mathrm{~km} \mathrm{~s}^{-1}$ and $38.2 \mathrm{~km} \mathrm{~s}^{-1}$ depending on the choosen spectral region. No periodic radial velocity variations are obvious in these data. The measured variations must be attributed partly to large scale surface motions and the fact that different measurements (wavelength regions) measure the $R V$ of different surface layers within a radially expanding outer atmosphere. Further, it must be considered that for the CES and FEROS measurements different radial velocity standard stars were employed for the cross correlation analysis. It is beyond the scope of this work to investigate these details. However, we estimate that the full amplitude of the orbital $R V$ variations for the red giant in MWC 560 must be smaller than $2.5 \mathrm{~km} \mathrm{~s}^{-1}$. Thus the maximum inclination for orbital velocities derived above is $i<16^{\circ}$ according to $\sin i<2.5\left[\mathrm{~km} \mathrm{~s}^{-1}\right] / 2 v_{\mathrm{rg}}$

Similarly, the $R V$ of the narrow emission lines is also practically constant at about $R V=+36 \mathrm{kms}^{-1}$. Unfortunately we found no measurements of narrow line $R V \mathrm{~s}$ in the literature with an accuracy of ours (Table 7). The comparison with the measurements of Tomov \& Kolev (1997) or Chentsov et al. (1997) indicates at least that possible orbital $R V$-variations must be smaller (full amplitude) than about $8 \mathrm{~km} \mathrm{~s}^{-1}$ for the narrow lines. Assuming that the narrow line region traces the orbit of the hot component yields the upper limit of $i<17^{\circ}$ for the inclination confirming but not improving the result above.

It can be expected that new high resolution measurments will improve significantly this result in the near future.

The size of the red giant. For the spectral type of the giant in MWC 560 we adopt M5.5 as derived by Meier et al. (1996) and Mürset \& Schmid (1999). This, together with empirical relations between radius and spectral type of e.g., Dumm \& Schild (1998) or van Belle et al. (1999) yields a radius of about $140 \pm 40 R_{\odot}(0.65 \pm 0.19 \mathrm{AU})$.

Distance and extinction. We need a distance to MWC 560 and the interstellar extinction for an estimate on absolute luminosities for the hot continuum emission.

A lower limit for the distance can be obtained from the radial velocities of interstellar absorption lines. MWC 560 has the galactic coordinates $l^{\mathrm{II}}=224^{\circ}, b^{\mathrm{II}}=+4^{\circ}$ where according to the maps of Brand \& Blitz (1993) the radial velocities of the interstellar medium is increasing with increasing distance. Both $\mathrm{Na}$ I and both CaII doublet lines 
Table 10. Parameters adopted for the MWC 560 jet system.

\begin{tabular}{|c|c|c|}
\hline parameter & value & (mainly) deduced from \\
\hline orbital period $P$ & $4.25,8.5 \mathrm{yr}$ & $\begin{array}{l}1990 \text { and } 1998 \text { obscu- } \\
\text { ration }\end{array}$ \\
\hline binary separation $a$ & $3-5.5 \mathrm{AU}$ & $\begin{array}{l}P \text {, typical masses for } \\
\text { symbiotic binaries }\end{array}$ \\
\hline $\begin{array}{l}\text { jet inclination } i_{\text {jet }} \\
\text { (orbit inclination) }\end{array}$ & $<16^{\circ}$ & $\begin{array}{l}P, a, \text { upper limits on } \\
\text { orbital } R V \text { variations }\end{array}$ \\
\hline red giant $r_{\mathrm{rg}}$ & $140 \pm 40 R_{\odot}$ & spectral type M5.5 III \\
\hline distance $d$ & $2.5 \pm 0.7 \mathrm{kpc}$ & $\begin{array}{l}r_{\mathrm{rg}}(\text { spectr. parallax), } \\
\text { IS line absorptions }\end{array}$ \\
\hline extinction $E_{B-V}$ & $0.15 \mathrm{mag}$ & $2200 \AA$ feature \\
\hline $\begin{array}{l}\text { hot component } L_{\mathrm{hc}} \\
\text { (for } 1998 / 99 \text { ) }\end{array}$ & $1000 L_{\odot}$ & $\begin{array}{l}d, \quad \text { measured flux, } \\
E_{B-V}\end{array}$ \\
\hline $\begin{array}{l}\text { accretion rate } \dot{M}_{\text {acc }} \\
{\left[M_{\odot} \mathrm{yr}^{-1}\right]}\end{array}$ & $\sim 5 \times 10^{-7}$ & $L_{\mathrm{hc}}, R_{\star}=0.01 R_{\odot}$ \\
\hline hot continnum $r_{\mathrm{hc}}$ & $\sim 3 R_{\odot}$ & $\begin{array}{l}L_{\mathrm{hc}},\left(T_{\text {eff }} \sim 20000 \mathrm{~K}\right) \\
\text { Stefan-Boltzmann law, }\end{array}$ \\
\hline $\begin{array}{l}\text { em. line region } r_{\mathrm{nl}} \\
{\left[R_{\odot}\right]}\end{array}$ & $\sim 10-30$ & $r_{\mathrm{nl}}>r_{\mathrm{hc}}$ \\
\hline $\begin{array}{l}\text { jet outflow rate } \dot{M}_{\text {jet }} \\
{\left[M_{\odot} \mathrm{yr}^{-1}\right]}\end{array}$ & $>7 \times 10^{-9}$ & $\begin{array}{l}r_{\mathrm{nl}}, \text { column density } \\
\text { and life time of jet ab- } \\
\text { sorptions }\end{array}$ \\
\hline
\end{tabular}

show the same absorption structure with 4 narrow components $F W H M \approx 7 \mathrm{~km} \mathrm{~s}^{-1}$ at $+14.1,+41.2,+49.1$ and $+66.3 \mathrm{~km} \mathrm{~s}^{-1}$. The last component is significantly weaker than the other components. According to Brand \& Blitz (1993) the features at $+41.2 \mathrm{~km} \mathrm{~s}^{-1}$ and $+49.1 \mathrm{~km} \mathrm{~s}^{-1}$ indicate a distance of $d \gtrsim 2.5 \mathrm{kpc}$ while the weak component at $+66.3 \mathrm{~km} \mathrm{~s}^{-1}$ must be due to a (small) absorption cloud with peculiar velocity. Tomov et al. (1992) attribute the (main) double component at $+41 /+49 \mathrm{~km} \mathrm{~s}^{-1}$ to the MWC 560 circumstellar region. However, the much higher resolution of our spectra show that this absorption is composed of very narrow components and that they are significantly red shifted with respect to the systemic velocity of $\approx 37 \pm 2 \mathrm{~km} \mathrm{~s}^{-1}$. This makes an association with a circumstellar (outflow) region unlikely.

The distance estimate is confirmed by the apparent IR-brightness of the red giant which indicates a distance of $d=2.5 \mathrm{kpc}$ (Meier et al. 1996). For this it was assumed that the M5.5 III giant in MWC 560 has a typical luminosity for its spectral type.

The interstellar extinction can be estimated from the broad UV absorption feature at $2200 \AA$. A rough analysis of the $2200 \AA$ depression in the IUE spectrum from April 29, 1990 plotted by Maran et al. (1991) suggests an extinction of $E_{B-V}=0.15 \pm 0.05$ mag. This spectrum is ideal for extinction estimates as it was taken when the continuum was bright and the jet absorptions weak (see Buckley 1992). A higher interstellar extinction $\left(E_{B-V}=0.23 \mathrm{mag}\right)$ was previously estimated from the IR colours of the red giant by Tomov et al. (1992) and Zhekov et al. (1996). For this they adopted a spectral type of M4.5 for the giant in MWC 560. Their method would also give a lower extinction if a spectral type of M5.5, as favoured by us, is adopted.

Based on these arguments we assume for our model of MWC 560 a distance of $2.5 \mathrm{kpc}$ and an extinction of $E_{B-V}=0.15 \mathrm{mag}$ (or $A_{V}=0.45 \mathrm{mag}$ ).

Luminosity and accretion rate of the hot continuum source. The luminosity in the hot continuum can now be determined with the distance and extinction derived above. The observed spectral energy distribution for the hot component in MWC 560 can be derived from published IUE spectra and photometry (e.g., Michalitsianos et al. 1991; Tomov et al. 1996). This has also been done by Panferov et al. (1997) for the maximum in April 1990 and a later phase where the brightness was lower. Dereddening the hot continuum spectrum with $E_{B-V}=0.15 \mathrm{mag}$ and integrating yields a total flux in the wavelength range 1000-8000 $\AA$ of $\approx 10^{-8} \mathrm{erg} \mathrm{s}^{-1} \mathrm{~cm}^{-2}$ for April 1990, about half to one third this value for 1992 and later, and about a forth for the pre-outburst IUE observation of 1984 . This translates with $d=2.5 \mathrm{kpc}$ into a luminosity of $2000 L_{\odot}$ for April 1990 and correspondingly lower values for the other dates. For our 1998/99 observations we adopt a total luminosity of $1000 L_{\odot}$ for the hot continuum. This accounts not only for the flux $\lambda>912 \AA$ but also for the ionizing radiation which is estimated to be about $200 L_{\odot}$ from the strengths of the $\mathrm{H}$ I lines (e.g., $F(\mathrm{H} \delta) \approx 2 \times 10^{-12} \mathrm{erg} \mathrm{s}^{-1} \mathrm{~cm}^{-2}$ dereddened line flux for 1998/99).

The luminosity of the hot continuum could be composed of 3 components: the accretion disk luminosity $L_{\mathrm{d}}$, the luminosity of the boundary layer $L_{\mathrm{bl}}$ and the intrinsic luminosity of the accreting star $L_{\star}$. We neglect in the following $L_{\star}$ assuming that $L_{\star} \ll 1000 L_{\odot}$. The reasons are that a white dwarf with high luminosity would produce highly ionized circumstellar emission lines what is not observed. On the other hand if the accreting star is luminous and has an extended, relatively cool photosphere then it would dominate the optical spectrum and the fast flickering activity must be attributed to unexpectedly large and fast changes in the photosphere. That the hot continuum is predominately due to accretion luminosity is not only supported by the fast flickering activity but also by the 1990 outburst characteristics. This outburst was coupled with strong changes in the jet outflow speed. The emission lines remained narrow and stayed at the systemic velocity indicating that no multi-directional (quasi-spherical) ejection or wind phase as for novae or similar thermonuclear events took place.

The flickering activity can be explained by accretion instabilities near the boundary layer (perhaps defined by the stellar magnetosphere) between disk and accreting 
object. The typical flickering time scale of $\sim 30$ min corresponds to an orbital period of gas in a Keplerian accretion disk at a distance of $r=0.25 R_{\odot}$ from the center of the accreting object with mass $M_{\mathrm{wd}}=0.5 M_{\odot}$.

Based on basic accretion disk theory (e.g. Pringle 1981; Warner 1995) the luminosities due to the accretion disk $L_{\mathrm{d}}$ and the boundary layer $L_{\mathrm{bl}}$ are to a first approximation equal:

$L_{\mathrm{bl}} \approx L_{\mathrm{d}} \approx \frac{G M_{\mathrm{wd}} \dot{M}}{2 R_{\star}}$,

where $G$ is the gravitational constant, $\dot{M}$ the mass accretion rate and $R_{\star}$ the radius of the accreting object. If we assume that $R_{\star} \approx 0.01 R_{\odot}$ as expected for a white dwarf (e.g. Provencal et al. 1998) then the accretion rate is about $\dot{M}=5 \times 10^{-7} M_{\odot} \mathrm{yr}^{-1}$ for the adopted accretion luminosity $\left(L_{\mathrm{bl}}+L_{\mathrm{d}}=1000 L_{\odot}\right)$.

Accretion onto an object $R_{\star}=0.01 R_{\odot}$ would produce a hard photon continuum with an effective temperature of $T_{\text {eff }}=\left(L_{\mathrm{bl}} / 4 \pi R_{\star}^{2} \sigma\right)^{1 / 4} \approx 3 \times 10^{5} \mathrm{~K}(\sigma$ : Stefan-Boltzman constant) emitting a large amount of hard photons in the EUV or soft X-ray region. But MWC 560 exhibits no emission lines from highly ionized gas indicating that reprocessing of radiation in the innermost accretion region must occur. A dense, spherical wind from the central region would be optically thick due to electron scattering $\left(\sigma_{\mathrm{e}}\right.$ : Thomson cross section) out to a radius $R_{\tau=1}=$ $\dot{M} \sigma_{\mathrm{e}} / 4 \pi m_{\mathrm{H}} v$. For a mass loss of $\dot{M}=10^{-7} M_{\odot} \mathrm{yr}^{-1}$ and an outflow velocity of $v=1000 \mathrm{~km} \mathrm{~s}^{-1}$ the photospheric radius is $R_{\tau=1}=0.9 R_{\odot}$. The corresponding effective temperature $T_{\text {eff }} \approx 30000 \mathrm{~K}$ is then compatible with the low ionization spectrum observed for MWC 560.

This interpretation implies that outflowing gas from the central accretion region has already a high velocity when it reaches the photosphere and becomes visible to the observer. Currently it is not clear, whether such a wind component is present among the different absorptions seen along the line of sight, and whether such an outflow from the central region could provide a significant fraction of the subsequently collimated jet gas. Thus, the jet gas could be due to a combination of outflows originating partly from the central optically thick region, and partly from the accretion disk as witnessed by the low velocity absoption component associated with region I in Fig. 19.

An alternative accretion disk model producing no hard photons involves a central star with a large radius $R_{\star} \sim 1 R_{\odot}$. But this would require for the observed bolometric luminosity an unrealistically high accretion rate of $>10^{-5} M_{\odot} \mathrm{yr}^{-1}$. Such a disk is much to bright in the visual region and the rotation time scale at the inner boundary is too long for explaining the fast flickering activity observed for MWC 560.

The radii of the continuum and the line emission regions. For simplicity we may assume that the continuum source is composed of two components: (a) a relatively small region around the accreting object where the luminosity of the boundary layer is emitted; and (b) the inner hot accretion disk radiating in the optical range. Thus, for the extent of the continuum source it is sufficient to know the size of the optically bright accretion disk. In principle this could be derived from standard accretion disk theory with a constant slope temperature structure $T(r) \propto r_{\mathrm{d}}^{-3 / 4}$ at large disk radii $r_{\mathrm{d}}$ and an integrated flux distribution of $F_{\lambda} \propto \lambda^{-2.33}$. However the spectral distribution of the hot continuum in MWC 560 is much flatter than this, more like $F_{\lambda} \propto \lambda^{-1}$. This points to a non-standard disk temperature profile caused e.g., by irradiation effects from the boundary layer, by the strong jet outflow, or another process.

We will not discuss non-standard disks here, although they should be investigated in future studies on MWC 560. For a very rough estimate it is sufficient to adopt a mean effective temperature $T_{\text {eff }}$ and calculate with the luminosity and the Stefan-Boltzmann law a characteristic emission radius. We can infer from the observed spectral energy distribution (and the absence of highly ionized emission lines, like He I) that the hot continuum comes predominantly from an optically thick region with a temperature of $T_{\text {eff }}=10000 \mathrm{~K}$ to $40000 \mathrm{~K}$. Given the estimated luminosity of $1000 L_{\odot}$ the radius of this emission region is between about $10 R_{\odot}$ for $10000 \mathrm{~K}$ and $0.65 R_{\odot}$ for $40000 \mathrm{~K}$. For our MWC 560 model we adopt a radius of $R_{\mathrm{hc}} \approx 3 R_{\odot}$ for the emitting region corresponding to a temperature of $T_{\text {eff }} \approx 20000 \mathrm{~K}$.

Determining the size of the narrow line emission region is crucial for estimates on the jet outflow rate, because we can measure observationally the size of the absorbing jet components relative to the size of the line emitting region of the accretion disk. Unfortunately it is difficult to determine the surface distribution of the line emission. It can be safely assumed from the strength of the emission lines that the narrow line region is significantly larger than the continuum source. Nonetheless it must be assumed that the line emission is peaked towards the disk center where the irradiation and excitation of the line emission is enhanced.

An emission measure can be obtained for the emission line region from the $\mathrm{H}_{\mathrm{I}}$ line fluxes, e.g., $F(\mathrm{H} \beta)=$ $6.5 \times 10^{-12} \mathrm{erg} \mathrm{s}^{-1} \mathrm{~cm}^{-2}$, estimated in Sect. 4.5. With a reddening $A_{V}=0.45\left(E_{B-V}=0.15\right)$ and a distance of $d=2.5 \mathrm{kpc}$ this translates into a $\mathrm{H} \beta$ luminosity of $L(\mathrm{H} \beta) \approx 2 L_{\odot}$ and an emission measure $\int N_{\mathrm{p}} N_{\mathrm{e}} \mathrm{d} V=$ $6 \times 10^{58} \mathrm{~cm}^{-3}$ assuming that $\mathrm{H} \beta$ is due to recombination radiation at $T_{\mathrm{e}} \approx 10000 \mathrm{~K}$. For the emitting volume a disk geometry with radius $R_{\mathrm{d}}$ and height $R_{\mathrm{d}} / 10$ is adopted. The emission measure derived above yields for the densities $N_{\mathrm{e}}=N_{\mathrm{p}}=10^{10}, 10^{11}$ or $10^{12} \mathrm{~cm}^{-3}$ the disk radii $R_{\mathrm{d}}=83 R_{\odot}, 19 R_{\odot}$ or $3.9 R_{\odot}$, respectively. Thus the $\mathrm{H}$ I and Fe II emission could originate from a relatively small part of the accretion disk. Therefore it seems to be reasonable to assume that a disk area with a radius of $\approx 10-30 R_{\odot}$ is covered by the major high velocity 
components of the jet absorbing about half of the narrow line flux.

Jet outflow rate. The Fe II jet absorptions show changes involving gas column densities of $N(\mathrm{H}) \approx 10^{23} \mathrm{~cm}^{-2}$ with time scales of roughly one to a few days (Sects. 3.1 and 4.7). This can be taken as measure for the replenished mass in the jet per unit area along the line of sight. However this rough estimate is based on events when strong changes in the jet absorptions occurred, i.e. when high velocity components appeared. Counting only the gas in the high velocity components may be quite reasonable, because they sweep up the slower moving gas in front of them. Thus the "quasi"-discrete (decelerating) jet gas absorptions may represent a major fraction of the jet gas near the source. Still, the relative contribution of the "steady" outflow between the high velocity events remains to be clarified. For this steady outflow there lacks the information how long the probed gas stream is and how much time it requires to replenish the gas. Thus it may be assumed as lower limit that a column density of $N(\mathrm{H}) \approx 10^{23} \mathrm{~cm}^{-2}$ is replenished in the jet at least for each strong high velocity outburst, which occur on a time scale of a week. Thus the minimum (mean) mass outflow column density is $N(\mathrm{H}) / \Delta t \approx 10^{17} \mathrm{~cm}^{-2} \mathrm{~s}^{-1}$.

For the total mass outflow we have to adopt a jet cross section $\pi R_{\text {jet }}^{2}$ corresponding to the determined mass outflow column density. As the strongly variable jet components are saturated for the $\mathrm{H}$ I transitions, they must cover practically the entire continuum source. We estimated above that the size of the continuum source is roughly $\sim 3 R_{\odot}$. The stronger high velocity components cover also about half of the narrow line emission, an area which was estimated to have a radius of the order $\approx 10-30 R_{\odot}$.

Adopting $R_{\text {jet }} \approx 10-30 R_{\odot}$ as jet radius probed by the Fe II absorptions yields together with the mass outflow column density estimated above a total mass outflow rate $\gtrsim 7-60 \times 10^{-9} M_{\odot} \mathrm{yr}^{-1}$. This result should be considered as lower limit because it accounts only for the outflowing mass seen due to the ejection by the high velocity components.

Thus, it is found that the jet outflow rate $\dot{M}_{\text {jet }}$ is at least one hundredth to one tenth of the mass accretion rate $\dot{M}_{\text {acc }}$ derived above. Different types of uncertainties are involved for the derivation of these quantities. For example $\dot{M}_{\text {acc }}$ depends on the adopted distance $d$ and the radius of the accreting object $R_{\star}$. However, if $\dot{M}_{\text {acc }}$ is higher, also the continuum and narrow line emission region will be larger and the resulting jet mass outflow rate will scale accordingly. Thus the ratio $\dot{M}_{\text {jet }} / \dot{M}_{\text {acc }} \gtrsim 0.01-0.1$ is less affected by uncertainties in the system parameters.

\section{Conclusions}

We present the results of an intensive monitoring program of the jet absorptions in the symbiotic system MWC 560.
In this work a general description of the data and the spectroscopic properties of the outflowing gas is given.

Many results of this study are new and have not been described in previous investigations of MWC 560. The characteristics and time scale of the strong jet absorption variability has been determined at least for the time covered by our campaign. Our data revealed the anticorrelation between the high velocity component and the shallow low velocity absorptions which proves their tight connection. Further the observations happened to show the end of an obscuration phase of the red giant by the jet gas probing the outflow at large distance from the source. It is also the first time that the fast, narrow absorption components of Ca II are described. In addition we investigated the properties of the narrow line emission. It is found that the allowed emission lines vary strongly like the hot continuum radiation and are obscured by the jet outflow. Thereby the transient high velocity components cover about half of the narrow line emission from the accretion disk while the continuous outflow at larger distance covers practically the entire narrow line region. Contrary to this the nebular lines (forbidden line transitions) are practically constant and not significantly absorbed by the jet gas.

Combining all this new information yields an outflow structure for the jet im MWC 560 described in detail in the previous section. The outflow can be characterized by three different regions along the jet axis: I. An initial acceleration region located mainly besides the accreting object above the accretion disk; II. A highly variable region extending to about one to a few AU showing the pulsed ejection and subsequent deceleration of high velocity material, whereby high velocity components appear in anticorrelation with the absorption from the initial acceleration region; III. A rather steady outflow region at larger distances. In addition we provide evidence that the fast, narrow Ca II absorptions can be attributed to the shocks generated by the collision of the pulsed high velocity gas ejections with slower moving jet gas.

For all these components we derive time scales, radial velocities, gas parameters and column densities and provide a geometric model and physical parameters for the MWC 560 jet outflow.

The obtained results demonstrate the enormous diagnostic possibilities offered by the MWC 560 system for the investigation of astrophysical jet outflows. Because the line of sight is practically parallel to the jet axis the outflowing gas can be studied as absorption against the underlying emission regions of the binary system. Small gas structures can therefore be followed in radial velocity space with high temporal resolution revealing the motion and physical properties of the gas in and near the acceleration region of the jet. To our knowledge, MWC 560 is the only stellar jet source known where this information can be obtained so easily from spectroscopic data. Also very favorable is the typical outflow velocity $\sim 1500 \mathrm{~km} \mathrm{~s}^{-1}$ and variability time scales $\sim 10^{3}-10^{6}$ s of the MWC 560 source 
because this range can be easily covered by available high resolution spectroscopy.

Due to these very advantageous properties of MWC 560, this system provides an important source of information for the investigation of astrophysical jets. It is often emphasized that basic parameters of jets from different sources obey simple scaling laws. For example the velocities of jet outflows scale roughly like the escape velocity from the accreting object $\propto\left(M_{\star} / R_{\star}\right)^{1 / 2}$ (see e.g., Pringle 1993; Blandford 1993; Camenzind 1997; Livio 1999). This suggests that the results found for MWC 560 could also be relevant for jet outflows in pre-main sequence stars, black hole binaries and active galactic nuclei. It is beyond the scope of this paper to discuss the results found for the outflow of MWC 560 in this more general context of astrophysical jets. However, we like to emphasize that MWC 560 is a most important object for studying the jet acceleration region close to the source. Therefore, the main result found in this study which is the velocity structure of the jet outflow with its pulsed acceleration and the subsequent deceleration, which generates shock features, and the termination in a rather smooth outflow could be a general property of astrophysical jets.

Acknowledgements. We are indebted to Toma Tomov who supplied a reduced Echelle spectrum of XX Oph. We thank Werner Schmutz (PMOD Davos) for discussions on radiative transfer issues, Markus Thiele (LSW Heidelberg) on jet hydrodynamics, and Andreas Korn (USM Munich) for support in our search for atomic data. HMS acknowledges financial support by the Deutsche Forschungsgemeinschaft (WO 296/27-1).

\section{References}

Allen, D. A. 1979, MNRAS, 186, 1

Arnaud, M., \& Rothenflug, R. 1985, A\&AS, 60, 425

Blandford, R. 1993, in Astrophysical jets, ed. D. Burgarella et al., STScI Symp. Ser., 6, 15

Bond, H. E., Pier, J., Pilachowski, C., Slovak, M., \& Szkody, P. 1984, BAAS, 16, 516

Brand, J., \& Blitz, L. 1993, A\&A, 275, 67

Buckley, D. A. H. 1992, IAU Symp., 151, 421

Camenzind, M. 1997, in Herbig-Haro flows and the birth of low mass stars, ed. B. Reipurt, \& C. Bertout (Kluwer), IAU Symp., 182, 241

Chentsov, E. L., Klochkova, V. G., \& Mal'kova, G. A. 1997, Bull. Spec. Astrophys. Obs., 43, 18

Dobrzycka, D., Kenyon, S. J., \& Milone, A. A. E. 1996, AJ, 111,414

Doroshenko, V. T., Goranskij, V. P., \& Efimov, Y. S. 1993, IBVS, 3824

Dumm, T., \& Schild, H. 1998, New Astron., 3, 137

Cowley, A. P., Schmidtke, P. C., Crampton, D., \& Hutchings, J. B. 1998, ApJ, 504, 854

Fuhr, J. R., Martin, G. A., Wiese, W. L., \& Younger, S. M. 1981, J. Phys. Chem. Ref. Data, 10, 305

Hamann, F. 1994, ApJS, 93, 485

Iben, I. Jr., \& Tutukov, A. V. 1996, ApJS, 105, 145
Iijima, T. 2000, ASP Conf. Ser., 204, 359

Ishioka, R., Uemura, M., \& Kato, T. 2001, IBVS, 5028

Joly, M. 1981, A\&A, 102, 321

Kaufer, A., Stahl, O., Tubbesing, S., et al. 1999, ESOMessenger, 95, 8

Kaufer, A., Stahl, O., Tubbesing, S., et al. 2000, in Optical and IR telescope instrumentation and detectors, ed. M. Iye, \& A. F. Moorwood, Proc. SPIE, 4008, 459

Keenan, P. C., \& McNeil, R. C. 1989, ApJS, 71, 245

Kolev, D., \& Tomov, T. 1993, A\&AS, 100, 1

Korista, K. T., Weymann, R. J., Morris, S. L., et al. 1992, ApJ, 401, 529

Kurucz, R. L., \& Bell, B. 1995, Kurucz CD-ROM No. 23, Smithonian astrophysical observatory (Cambridge, Mass)

Lamers, H. J. G. L. M., \& Cassinelli, J. P. 1999, Introduction to stellar winds (Cambridge University Press)

Lee, H. W. 2000, ApJ, 541, L25

Livio, M. 1999, Phys. Rep., 311, 225

Luthardt, R. 1991, IBVS, 3563

Maran, S. P., Michalitsianos, A. G., Oliversen, R. J., \& Sonneborn, G. 1991, Nature, 350, 404

Margon, B. 1984, ARA\&A, 22, 507

Merrill, P. W., \& Burwell, C. G. 1943, ApJ, 98, 153

Michalitsianos, A. G., Maran, S. P., Oliversen, R. J., et al. 1991, ApJ, 371, 761

Michalitsianos, A. G., Perez, M., Shore, S. N., et al. 1993, ApJ, 409, L53

Mikolajewski, M., Tomov, T., Georgiev, L., \& Jones, A. 1997, IBVS, 4519

Mirabel, I. F., \& Rodriguez, L. F. 1999, ARA\&A, 37, 409

Mundt, R., \& Fried, J. W. 1983, ApJ, 274, L83

Mürset, U., \& Schmid, H. M. 1999, A\&AS, 137, 473

Mürset, U., Dumm, T., Isenegger, S., et al. 2000, A\&A, 353, 952

Meier, S. R., Rudy, R. J., Lynch, D. K., et al. 1996, AJ, 111, 476

Netzer, H., \& Wills, B. J. 1983, ApJ, 275, 445

Netzer, H. 1988, in Physics of formation of Fe II lines outside LTE, ed. R. Viotti, A. Vittone, \& M. Friedjung, IAU Coll. 94 (Reidel, Dordrecht), 247

Nussbaumer, H., Schmid, H. M., \& Vogel, M. 1989, A\&A, 211, L27

Panferov, A. A., Fabrika, S. N., \& Tomov, T. 1997, Bull. Spec. Astrophys. Obs., 43, 37

Pringle, J. E. 1981, ARA\&A, 19, 137

Pringle, J. E. 1993, in Astrophysical jets, ed. D. Burgarella et al., STScI Symp. Ser., 6, 1

Provencal, J. L., Shipman, H. L., Hog, E., \& Thejll, P. 1998, ApJ, 494, 759

Sahai, R., \& Nyman, L.-A. 2000, ApJ, 537, L145

Sanduleak, N., \& Stephenson, C. B. 1973, ApJ, 185, 899

Savanov, I. S., Huovelin, J., \& Tuominen, I. 1990, A\&AS, 86, 531

Schild, H., Dumm, T., \& Mürset, U. 2001, A\&A, 366, 972

Schmid, H. M. 2001, in $\eta$ Car and other mysterious stars, ed. T. R. Gull et al., ASP Conf. Ser. (in press)

Shore, S. N., Aufdenberg, J. P., \& Michalitsianos, A. G. 1994, AJ, 108, 671

Sigut, T. A. A., \& Landstreet, J. D. 1990, MNRAS, 247, 611

Spitzer, L. 1978, Physical processes in the interstellar medium (Wiley, New York)

Stone, J. M., \& Norman, M. L. 1993, ApJ, 413, 210

Taylor, A. R., Seaquist, E. R., \& Mattei, J. A. 1986, Nature, 319,38 
Tomov, T., \& Kolev, D. 1997, A\&AS, 122, 43

Tomov, T., Kolev, D., Georgiev, L., et al. 1990, Nature, 346, 637

Tomov, T., Zamanov, R., Kolev, D., et al. 1992, MNRAS, 258, 23

Tomov, T., Kolev, D., Munari, U., Sostero, G., \& Lepardo, A. 1995, A\&A, 300, 769

Tomov, T., Kolev, D., Ivanov, M., et al. 1996, A\&AS, 116, 1

Tomov, T., Kolev, D., \& Munari, U. 1997, in Physical processes in symbiotic binaries, ed. J. Mikolajewska (Copernicus Found. for Polish Astr., Warsaw), 197

van Belle, G. T., Lane, B. F., Thompson, R. R., et al. 1999, AJ, 117, 521
Verner, E. M., Verner, D. A., \& Korista, K. T. 1999, ApJS, 120,101

Viotti, R., Vittone, A., Friedjung, M., (eds.) 1988, Physics of formation of Fe II lines outside LTE, IAU Coll. 94 (Reidel, Dordrecht)

Warner, B. 1995, Cataclysmic variable stars (Cambridge Univ. Press)

Wiese, W. L., Smith, M. W., \& Glennon, B. M. 1966, Atomic Transition Probabilities, vol. I, NSRDS-NBS 4

Wiese, W. L., Smith, M. W., \& Glennon, B. M. 1969, Atomic Transition Probabilities, vol. II, NSRDS-NBS 22

Zhekov, S. A., Hunt, L. K., Tomov, T., \& Gennari, S. 1996, A\&A, 309, 800 